Manuscript Number: ENGSTRUCT-D-16-00147R1

Title: On the Dynamic Response of Square Tunnels in Sand

Article Type: Research Paper

Keywords: Square tunnels; Dynamic centrifuge testing; Dynamic analysis; Interface conditions; Tunnel rigidity

Corresponding Author: Dr. Grigorios Tsinidis,

Corresponding Author's Institution: Aristotle University

First Author: Grigorios Tsinidis

Order of Authors: Grigorios Tsinidis; Kyriazis Pitilakis; Gopal Madabhushi

Abstract: The paper investigates the seismic response of square tunnels in sand by means of dynamic centrifuge testing and numerical analysis. A series of dynamic centrifuge tests conducted at the University of Cambridge on a square aluminium model tunnel embedded in dry sand, are initially presented. The tests, which were designed in order to investigate the seismic response of flexible tunnels, are analyzed numerically be means of full dynamic analysis of the coupled soil-tunnel system, using different soil and soil-tunnel interface models. Numerical predictions are compared to the experimental data, in order to better understand the response mechanism and validate the numerical modelling. The validated numerical models are then used to investigate the effect of the lining rigidity on the soil-tunnel system dynamic response. The experimental and numerical results reported herein, indicate a nonnegligible rocking deformation mode for the tunnels coupled with racking distortion during seismic shaking. The significant effects of the lining rigidity, soil-tunnel interface characteristics and soil yielding on the dynamic earth pressures and the shear stresses developed around the perimeter of the tunnel, as well as on the dynamic lining forces, are also reported and discussed. 


\section{Response to Reviewers Comments}

The Authors gratefully acknowledge the constructive criticism of the Reviewers and their useful comments and suggestions, which helped to further improve the clarity and quality of the paper. Major modifications in the revised manuscript are highlighted in yellow. Below are the Authors' replies to individual comments:

\section{Reviewer \#1:}

\section{Reviewer's comment:}

The paper studies the seismic response of square tunnels combing experimental and numerical methods. The numerical analyses are validated against centrifuge-model tests, and the validated models are used to conduct a parametric study. The paper is well written and the subject is of interest to the readership of the journal. The paper should be published after the following drawbacks are addressed:

1) The beginning of the introduction gives the impression that tunnels are very sensitive to seismic shaking. Although there are cases of severe damage or collapse of tunnels in strong earthquakes, as correctly mentioned by the authors, tunnels are in general not so sensitive to seismic shaking. The introduction should be revised, so as not to give a wrong impression to the readers.

\section{Authors' reply:}

The Authors agree with the Reviewer. Tunnels were found less vulnerable to seismic shaking compared to above ground structures during recent strong earthquakes. To avoid any misunderstanding the introduction has been revised as follows:

Pages: 1-2, lines: 31-33, 1-2

"Underground structures and tunnels behaved better than above ground structures during recent strong earthquakes. However, several cases of severe damages to total collapse have been reported in the literature (e.g. [27, 53]). Shallow embedded structures in soft soil were found more vulnerable to seismic shaking, while the vulnerability was generally increased in cases where seismic design provisions were not encountered."

\section{Reviewer's comment:}

2) The description of the physical model requires some improvement. For example, it would be nice to provide more details on the Hostun HN31 sand. If this can be found in previous publication(s), appropriate reference would suffice. Also, a brief description of the position sensors would be very helpful for non-specialist readers.

\section{Authors' reply:}

The Authors provide in the manuscript a diagram of the particle size distribution of Hostun HN31 sand (Figure 1a) along with the main physical properties of the sand fraction (Table 1). The above characteristics were derived from laboratory tests on the specific sand fraction, carried out in Aristotle University of Thessaloniki. A relevant reference is provided in the manuscript.

With reference to the position sensors, these sensors were actually string potentiometers. They were connected to the tunnel through small cables that were attached to the side-walls using small screws. The following comment has been added in the revised manuscript: 
Page: 5, lines: 6-9

"Two string potentiometers (POTs in Fig. 3) were attached to the tunnel to monitor its vertical displacement and possible rocking during shaking. The above instruments were connected to the tunnel through small cables that were attached to the side-walls using small screws."

\section{Reviewer's comment:}

3) In section 2.6, it is stated that the results were filtered using an 8th order Butterworth filter. Why was the specific filter selected and how sensitive are the results to the applied filtering technique?

\section{Authors' reply:}

The particular filter was selected as a reliable filter which is used for many years in experimental campaigns carried out in the University of Cambridge. The cut off frequencies were set at $10-400 \mathrm{~Hz}$ covering the frequency range of interest. A discussion on filtering techniques used in processing centrifuge data can be found in Madabhushi (2014) (the reference [35] in the revised manuscript). The Authors agree that the order of the filter may probably affect the results to some extent but it is necessary to avoid high frequency parasitic pulses. To avoid potential deviations between the numerical and the experimental results due to this effect, a similar filtering procedure was applied also on the numerical results.

\section{Reviewer's comment:}

4) In page 8, it is stated that the 3D analysis was performed assuming full bonding between the tunnel and the soil, as this matched better the results of the experiments. This is a bit counter-intuitive. The tensionless interface should provide better results, as it is more realistic. If the bonded interface yields a better prediction, then this could be due to other factors that have not been taken into account. Please explain.

\section{Authors' reply:}

The Reviewer's concern is relevant. In the Authors view, the rather worse performance of the tensionless interface compared to full bonding conditions should be attributed to the numerical simulation per se. Owing to the high flexibility of the lining, the lining elements exhibits large inward deformations (deformations towards the cavity) during the introduction of the gravity loads (i.e. static step of the analysis). The soil elements, surrounding the tunnel, can not follow this deformation, due to artificial 'arching effects' related to the simulation of the soil as a continuum. In this context, a small 'artificial gap' between the soil and the tunnel elements is created for the cases where a tensionless interface is considered. This behavior opposes to the actual behavior in the centrifuge, where no separation between the sand and the lining could occur. The 'artificial gap' was more evident for the deep tunnel (Test 2), as the confining pressures and the associated lining inward deformations were higher in this case. The analyses under full bonding conditions ensured that the soil elements were following the deformation of the lining elements, thus providing numerical predictions closer to the recorded data. It is noteworthy that this numerical weakness is expected to be reduced with the increasing lining rigidity. To clarify this issue, the following comment has been added in the description of the numerical models:

Page: 8 , lines: 9-10

"The better performance of full bonding conditions in the particular test case is discussed in the ensuing." 
In addition, the text referring to the earth pressures has been revised in the manuscript, as follows:

Page: 18, lines: 4-18

"The better performance of the no-slip full bonding conditions in Test 2 should be attributed to the numerical simulation per se. Owing to the high flexibility of the lining, the lining elements exhibits large inward deformations (deformations towards the cavity) during the introduction of the gravity loads (i.e. static step of the analysis). The soil elements, surrounding the tunnel, are not capable to fully follow this deformation, due to a kind of artificial 'arching effect' related to the simulation of the soil as a continuum. In this context, a small 'artificial gap' is created between the soil and the tunnel elements for the cases where an interface is considered. This numerical behavior opposes to the actual behavior in the centrifuge, where no separation between the sand and the lining could occur. The gap was more evident for the deep tunnel (Test 2), as the confining pressures and the associated lining inward deformations were higher in this case. The analyses under full bonding conditions ensured that the soil elements were following the deformation of the lining elements, thus providing numerical predictions closer to the recorded data. It is noteworthy that the above numerical weakness is expected to be reduced with the increasing lining rigidity."

\section{Reviewer's comment:}

5) The description of the elasto-plastic model requires substantial improvement. If this is a model developed by the authors, then a more detailed description is necessary, including the failure criterion, the hardening law, etc. If the authors are using a model available in abaqus or previously developed, then appropriate reference would suffice. In one of their previous publications, the authors had compared the efficiency of various constitutive models. Is this model the same with one of the previous models? If it differs, please explain the key differences.

\section{Authors' reply:}

A first series of analysis were conducted using an elastic model with viscous damping (i.e. visco-elastic model). The soil stiffness, corresponding to the effective degraded stiffness, as well as the viscous damping, were back-calculated, by comparing the predictions of 1D equivalent linear soil response analyses with the recorded acceleration at the soil 'free-field'. The procedure is discussed in detail in section 3.2 of the revised manuscript. In the second series of analyses, the above model was combined with a Mohr-Coulomb model, so as to account for the yielding response of the soil. The sand equivalent properties (i.e. effective stiffness and viscous damping) were kept the same as in the visco-elastic analyses. The adopted soil constitutive models were selected as they are proposed in guidelines for the dynamic analysis of embedded structures and are commonly used by the tunnelling design practise due to their easy calibration and control. Actually, the visco-elasto-plastic model, which was implemented in the final series of analysis, has been recently used by Cilingir and Madabhushi for the simulation of similar dynamic centrifuge tests on model tunnels in dry sand, revealing reasonable comparisons between the recorded data and the numerical results (references [16-18] in the revised manuscript). To clarify this crucial aspect, the presentation of the implemented constitutive models has been revised in the manuscript, as follows:

Page: 8 , lines: 20-32

"The soil response under seismic shaking was simulated in two ways. In a first series of analyses, a visco-elastic model was implemented, introducing an effective sand shear modulus distribution and viscous damping (i.e. following the equivalent linear approximation 
method). The sand equivalent properties were back-calculated, as discussed in the following section. In the second series of analyses, a non-associated Mohr-Coulomb yield criterion was combined with the above visco-elastic model to account for the permanent deformations of the soil. To implement the latter model herein, the sand equivalent properties were set equal to those of the visco-elastic model. This elasto-plastic approach has been recently used by Cilingir and Madabhushi for the simulation of similar dynamic centrifuge tests on model tunnels in dry sand, revealing reasonable comparisons between the recorded data and the numerical results [16-18]. The above models were selected as they are proposed in guidelines for dynamic analysis of embedded structures (e.g. equivalent linear approximation in [20]) and are commonly used in tunnelling design practice due to their easy calibration and control."

\section{Reviewer's comment:}

6) In page 16, the authors try to explain why the unbonded interface matches the results better (see also previous comment), something which is attributed to the increase of the confining pressures. But the confining pressures are taken into account by a Coulonb-type interface, so why shouldn't it provide good results? The explanation offered by the authors is not convincing and should be revised.

\section{Authors' reply:}

Please see the Authors' response to Comment \#3. The explanation has been revised in the manuscript, in order to avoid misunderstands.

\section{Reviewer's comment:}

7) In Fig. 16, there is a non-negligible difference between analysis and experiments in terms of the residual stressing of tunnel members. Is this a problem of the analysis or of the experimental measurement? Was the residual deformation of the tunnel substantial in the experiments?

\section{Authors' reply:}

No residual deformation was reported for the tunnel lining after testing. As it is stated in the manuscript, the post-earthquake residual response is attributed to the densification and yielding of the sand around the model tunnel during shaking. This response is in line with recent findings of Lanzano et al. (reference [33] in the revised manuscript), who performed similar dynamic centrifuge tests on a circular model tunnel embedded in sand. To the Authors point of view, the observed differences between numerical predictions and the recorded data should be attributed to several issues, including the relative simplification and calibration of the numerical modelling, as well as uncertainties related to the preparation of the model and the calibration of the strain gauges. More specifically, the exact properties of sand in the area close to the tunnel are practically not well known, as the unavoidable 'shadow and silo' effects during the formation of the centrifuge model (i.e. sand pouring performed from a height), resulted in a lower densification level of this area compared to 'free-field' conditions. The successive shaking should have probably densified this area. However, the extent of this densification is unknown. This issue is discussed in section 3.2 of the revised manuscript. The above situation affects the mechanical properties of the sand and the sand-tunnel interface, making the calibration of the constitutive models cumbersome. The above problems related to the determination of the constitutive parameters may explain part of the differences between the numerical and the experimental results. Moreover, the calibration procedure was done carefully following the comprehensive procedure described in Tsinidis et al. (2016b). However, there are always some uncontrollable uncertainties related with the above 
procedure, potentially causing some differences with the numerical results. Accounting for the complexity of the problem, the comparisons between the experimental and the numerical results seem to be fair enough. The following comments have been added in the revised manuscript to address this issue:

Page: 14 lines: $10-17$

"The numerical predictions are in a fair agreement with the recorded data (at least in order of magnitude). The observed differences between the recorded and the computed data should be attributed to the several issues, including the relative simplification and calibration of the constitutive model, as well as uncertainties related to the calibration of the strain gauges. As mentioned above, the properties of the sand in the area around the tunnel were affected by the 'shadow and silo' effects during the model formation, making the calibration of the constitutive model cumbersome. Although the calibration of the strain gauges was done very carefully (i.e. [51]), there are always some unavoidable uncertainties related with the calibration of the above instruments, potentially causing some differences between the experimental and numerical results."

Page: 24, lines: 7-10

"The deviations between the experimental data and the numerical predictions should be attributed to the simplification of the constitutive model, to calibration issues of the above model, as well as to uncertainties related to the calibration of strain gauges."

\section{Reviewer's comment:}

8) Typos etc.:

* Page 2: "seismic design for underground" should be "seismic design of underground"

* Page 5: "As regards the strain gauges" should better be "Regarding the strain gauges"

* Page 20: "For sake brevity" should be "For the sake of brevity"

* Page 21: "are well compared" should be "compare well"

Authors' reply:

All the typos have been corrected in the revised manuscript.

\section{Reviewer \#2:}

\section{Reviewer's comment:}

The manuscript investigates the dynamic response of flexible square tunnels in dry sand by means of dynamic centrifuge testing and rigorous numerical analysis. The effects of the lining stiffness, of the soil-tunnel interface characteristics and of the soil yielding are taken into account.

The research work is interesting however some issues should be addressed before the paper could be accepted for publication, as detailed in the following.

1) It is not very clear why the Authors have performed $3 D$ numerical modelling of Test 2, while most of the numerical analyses were performed in plane strain. The centrifuge model is clearly designed to represent a plane strain condition, hence $2 D$ analyses should be enough accurate to reproduce the experimental conditions. Why should they expect "possible $3 D$ effects" as stated in section 4 (2D versus $3 D$ analysis)? Could this section be dropped? 


\section{Authors' reply:}

The Reviewer's concern is relevant. Indeed, the centrifuge model was designed to simulate plane strain conditions. In this context, a 2D plane strain numerical analysis is accurate enough to reproduce the recorded response. This is actually verified by the comparisons between the numerical predictions and the experimental data. The issue of the potential 3D effects on the response was raised by a Reviewer in a relevant publication of some of the Authors, in which dynamic centrifuge tests on a circular tunnel were analysed (Tsinidis et al., 2013, Numerical simulation of round robin numerical test on tunnels using a simplified kinematic hardening model, Acta Geotechnica 9(4): 641-659). Therefore, it was decided to perform the $3 \mathrm{D}$ analysis, so as to have this comparison for future reference regarding this type of analysis.

\section{Reviewer's comment:}

2) About the strength parameters: the Authors state that "friction angle $\varphi$ was set equal to $33^{\circ}$ (critical friction angle for specific sand fraction), while the dilatancy angle $\psi$ was assumed equal to $3^{\circ}$ ". The latter choice seems to be independent from the relative density of the sand, that is quite different between test 1 and test $2(51 \% \mathrm{vs} .89 \%)$. While the critical friction angle is independent from density, dilatancy angle and peak friction angle are not. The Authors should comment on this.

\section{Authors' reply:}

The exact properties of sand in the area close to the tunnel are practically not well known. Indeed, the unavoidable 'shadow and silo' effects during the formation of the centrifuge model (i.e. sand pouring performed from a height) resulted in a lower level of densification for the sand in this area compared to the 'free-field' conditions. The successive shaking should densify to a certain degree this area. However, the extent of this densification is not known. This issue is discussed in section 3.2 of the revised manuscript. This situation, which was evident in both test cases, affected the mechanical properties of the sand around the tunnel, which are of particular importance for prediction of the tunnel response. In this context, it was decided to run the numerical analyses, by assuming the sand strength properties, so as to be compatible with a less densified sand area around the tunnel. The following comment has been added in revised manuscript:

Page: 11, lines: 27-31

"The above strength parameters were selected, so as to be compatible with the looser conditions of the sand area around the tunnel, which are crucial for the tunnel response. As mentioned in section 3.2, the densification level of this area is reduced compared to the "freefield' due to the 'shadow and silo' effects, associated with the formation of the sand model."

\section{Reviewer's comment:}

3) Static response. By looking at the static values of bending moments and axial forces (Tables 4 and 5), differences between 50\% and 100\% (see SG-B2) can be observed between measured and calculated bending moments. The investigated effect of interface conditions is unable to explain such a difference, in about one half of the cases. The reasons claimed by the Authors ("The observed differences should be attributed to the simplification of the implemented constitutive model, as well as to the inevitable uncertainties related with the estimation of the soil, the tunnel and the soil-tunnel interface mechanical characteristics ") are non completely convincing: for instance numerical analyses predict the same value of axial force for the symmetrical positions of $S G-A 1$ and $S G-A 3$, while the corresponding experimental measurements differs by a factor of 2 . 


\section{Authors' reply:}

Indeed large differences can be observed in some cases between the numerical predictions and the recorded data. In the Authors point of view, the deviations should be attributed to the relative simplification and calibration of the numerical modelling, as well as to uncertainties related to the preparation of the model and the calibration of the strain gauges. As stated in the response to Comment \#3, the 'shadow and silo' effects during the formation of the centrifuge model affected the mechanical properties of the sand around the tunnel, making the calibration of the constitutive models cumbersome and thus affecting the numerical predictions. This may explain part of the differences between the numerical and the experimental results. Moreover, the calibration procedure was done carefully following the procedure outlined in Tsinidis et al. (2016) (reference [51] in the revised manuscript). However, there are always unavoidable uncertainties related with the above procedure, potentially causing some differences with the numerical results. Actually, the large deviation in the recorded axial forces in the symmetrical positions SG-A1 and SG-A3 is related to the large difference observed in the calibration factors of the particular strain gauges ([51]). Accounting for the complexity of the investigated problem, the comparisons between the experimental and the numerical results seem to be fair enough. The particular section has been revised in the manuscript as follows:

Page: 14, lines: 8-18

"The numerical predictions are in a fair agreement with the recorded data (at least in the order of magnitude). The observed differences between the recorded and the computed data should be attributed to the several issues, including the relative simplification and calibration of the constitutive model, as well as uncertainties related to the calibration of the strain gauges. As mentioned above, the properties of the sand in the area around the tunnel were affected by the 'shadow and silo' effects during the model formation, making the calibration of the constitutive model cumbersome. Although the calibration of the strain gauges was done very carefully (i.e. [51]), there are always some unavoidable uncertainties related with the calibration of the above instruments, potentially causing some differences between the experimental and the numerical results."

\section{Page: 15 lines: 2-4}

"The large deviation between the recorded axial forces in the symmetrical positions SG-AI and $S G-A 3$ is related to the large difference observed in the calibration factors of the particular strain gauges [51].'

\section{Reviewer's comment:}

4) When commenting Fig. 10 the Authors stated that "the computed earth pressures around the tunnel are clearly affected by the soil-tunnel interface characteristics. For the deep tunnel case (Test 2), the numerical predictions for fully bonding conditions are closer to the experimental records. On the contrary, full-slip interface conditions provide results that are better compared to the recorded data for the shallow tunnel (Test 1)". However, when looking at the figure in all cases the residual value of pressure predicted by numerical models in both interface conditions is very similar but far from the measured one. In my opinion the interface conditions only affect the reversible changes of pressure during shaking. In deed, when looking at the amplitude of cycles only, the matching between numerical predictions for fully bonding (full slip) conditions and the experimental results for the deeper (shallower) tunnel is more evident. Similar conclusions can be drawn from figure 12. The Authors should better highlight this in the text. 


\section{Authors' reply:}

The comment is appreciated. Indeed, the effect of the interface conditions is more evident in terms of reversible changes of the pressures during shaking. However, it still exists in terms of residual values (i.e. comparisons referring to PC1 for Test 1 in Fig. 10a) due to the effect of the interface conditions on the yielding response of the surrounding ground (i.e. Fig.13). The relevant comment has been revised in the manuscript as follows:

Page: 17, lines: 22-24

"The computed earth pressures around the tunnel are clearly affected by the soil-tunnel interface characteristics, with the effect being generally more evident in the reversible component of the earth pressure time histories."

The observed differences between the recorded and the computed earth pressures may be attributed to several issues, including the calibration of the mechanical properties of the sand (i.e. at the vicinity of the tunnel), the assumptions made for the soil constitutive model, as well as issues related to the miniature earth pressures cells recording capabilities in the case of granular dry sand. All these aspects are discussed in the revised manuscript.

\section{Reviewer's comment:}

5) When commenting Figure 14 the Authors state that "The dynamic part of the earth pressures computed by the simplified approaches and in particular with the prescriptions of the EC8 for non deformable rigid walls [11] are well compared with the numerical results in terms of magnitude". This is very different from my perception from Fig. 14b. This must be clarified.

\section{Authors' reply:}

The comment is relevant. This state has been revised in the manuscript as follows:

Page: 22, lines: 8-12

"The earth pressures (dynamic part) computed following the prescriptions of the EC8 for non deformable rigid walls [11] are more conservative than the M-O approach. However, the results of both simplified approaches deviate considerably from the numerical predictions. Evidently, the simplified approaches are not capable to reproduce the actual earth pressures distribution pattern (e.g. concentrations of the earth pressures at the corners)."

\section{Reviewer's comment:}

6) On lines 23 to 25 on page 22 the Authors state that: "it is expected that the soil-tunnel interface characteristics will affect the computed bending moments both in terms of residuals and dynamic increments". This may appear in contradiction with Figure 10 on dynamic soil pressure (see comment \#4). However, as the Authors observe, different yielding induced by different interface conditions may also affect the sand stiffness around the tunnel, thus influencing the lining internal forces (Fig. 16) more than the acting soil pressures (Fig 10). This should be highlighted in the section and in the conclusions.

\section{Authors' reply:}

Similar to the dynamic earth pressures, the effect of interface characteristics is more evident in the reversible component of the dynamic lining bending moments, but it still exists for the residual component, due to the effect of the interface characteristics on the soil yielding 
response around the tunnel and thus on the stiffness of the soil. The following comment is added in the revised manuscript:

Page: 25, lines: 2-6

"The soil-tunnel interface characteristics affect the soil yielding (i.e. Fig. 13) and to some extent the sand stiffness around the tunnel. In this regard, it is expected that the soil-tunnel interface characteristics will affect the computed bending moments both in terms of residuals and dynamic increments. The effect of interface conditions is generally higher for the dynamic bending moment compared to the dynamic earth pressures."

\section{Reviewer's comment:}

7) Section 5.2.5 presents very interesting results in terms of the effect of lining stiffness and soil-tunnel interface characteristics (associated to soil yielding) on the internal forces arising in the tunnel during and after shaking. However the presentation of the results is quite long and the six figures in this section very dense of information. I believe that a paragraph that initially anticipates the main findings would be useful to the reader at the beginning of this section. The Authors might also consider revising the whole section and using subsections.

\section{Authors' reply:}

Following the Reviewer's suggestion, the section has been divided in to two subsections, referring to the dynamic bending moments and the dynamic axial forces. In addition, a paragraph has been added in the beginning of the section summarizing the main findings.

Page: 23, lines: 21-29

"This section summarizes the main findings regarding the dynamic lining forces of the tunnels, which are crucial for the detailing of the lining sections. The crucial effects of soiltunnel relative flexibility, soil-tunnel interface conditions and soil yielding on the dynamic lining forces are highlighted and discussed. In line with the experimental data, the numerical results reveal post-earthquake residual values for the lining forces of the flexible tunnels. The increase of the lining rigidity results in a significant reduction of this permanent response. Soil-tunnel interface characteristics are found to affect significantly the dynamic axial forces, while the effect is less evident for the dynamic bending moment. These observations are presented in detail in the following paragraphs."

\section{Reviewer \#3:}

\section{Reviewer's comment:}

The paper presents a set of centrifuge tests and numerical analyses of the seismic response of square tunnels immersed in a sandy soil. The topic is of interest for a wide audience and deserves research insights as it is not yet well understood and properly dealt with in the engineering practice.

The originality of the paper is by no means in the experimental results, which are well conducted and reasonably well discussed. The weak point of the work is in the FE numerical analyses, which are not described and discussed in a sufficient detail to make them useful to interpret and generalise the experimental observations. In fact, the related set up and calibration procedures of the adopted constitutive models are only barely discussed, leading to an overall impression of a simple fitting exercise which systematically leads to the best estimate of the experimental observations, those latter being known a priori. Surprisingly, given the well-known experience of two of the Authors, the quality of the text is not consistently good throughout the paper, making necessary a careful revision by an English speaking reader. 
In light of what above, I propose the Authors to substantially revise the paper and resubmit it only after a significant improvement of the FE related parts will be achieved.

In the following, some more detail comments are summarised.

\section{Authors' reply:}

The Authors gratefully acknowledge the constructive criticism of the Reviewer, which helped to further improve the clarity and quality of the paper. The paper aims at shed light on several aspects of the dynamic response of rectangular tunnels in soft soil, using results from two dynamic centrifuge tests that were performed on a scaled square model tunnel embedded in dry sand. Numerical analyses are conducted on the basis of a Class $\mathrm{C} 1$ prediction, so as to better understand the response mechanism and validate the numerical models. In this context, the constitutive models are calibrated through back-analysis of the tests. The validated numerical models are then employed to perform additional analyses, so as to further investigate the seismic response of rectangular tunnels, focusing on the effect of the lining rigidity. Following the Reviewer's suggestions, the Authors have revised several parts of the manuscript, describing better the FE analyses and presenting in more detail their selections. A thorough revision of manuscript has been also made so as to improve the language. Below are the Authors' replies to individual comments.

\section{Reviewer's comment:}

Page 3, line 13: emax, emin, d10,50,60 are not mechanical parameters but rather physical ones. Please modify the sentence.

\section{Authors' reply:}

The sentence has been modified according to the Reviewer's suggestion.

\section{Reviewer's comment:}

Page 3, line 24: the adopted thickness of the model is equivalent to $0.13 \mathrm{~m}$ : this really is far thinner than what used in real cases: the Authors should discuss this limitation in a more clear and fair manner.

\section{Authors' reply:}

The concern of the Reviewer is relevant. This thickness is unrealistic in practice, as the design analysis for the static loads would have resulted in a much thicker lining. However, this selection was made in order to study the effect of high flexibility on the tunnel response, as well as to obtain clear measurements of the lining bending and axial strains. It is worth noticing that the soil-tunnel relative flexibility may change significantly along the longitudinal axis of a tunnel, which passes through various geological conditions. In this context, it is interesting to investigate the response of tunnel at an extreme flexibility condition. To avoid misunderstandings the particular section has been revised as follows:

Pages: 3-4, lines: 24-26, 1

"This thickness is unrealistic in practice, as the design analysis for the static loads would have resulted in a much thicker lining. However, this selection was made in order to study the effect of high lining flexibility on the tunnel response, as well as to obtain clear measurements of the lining bending and axial strains."

\section{Reviewer's comment:}

Page 7, line 8: the Authors are asked to provide quantitative details on the mesh element size and its relation to the wave length of the signals. 


\section{Authors' reply:}

The element size in both 2D and 3D models was selected in a way that ensured the efficient reproduction of the waveforms of the whole frequency range under study (i.e. $0.2-8 \mathrm{~Hz}$ in prototype scale). In particular, the maximum size of the elements was set equal to 0.6 and 1.0 $\mathrm{m}$ for the $2 \mathrm{D}$ and the $3 \mathrm{D}$ analyses, respectively, while the mesh was generally finer near tunnel. This information has been added in the revised manuscript.

\section{Reviewer's comment:}

Page 8, line 10: what equivalent thickness corresponds to the model $10 \mathrm{~mm}$ in the prototype? Please add quantitative information on that.

\section{Authors' reply:}

The equivalent concrete lining thickness in this case is equal to $0.70 \mathrm{~m}$ (assuming the elastic modulus for equivalent concrete section $\mathrm{E}=30 \mathrm{GPa}$ ). According to Wang ([53] in the revised manuscript), the corresponding flexibility ratio, $F$, is equal to 0.29 . To this end, the tunnel may be characterized as rigid. This information has been added in the revised manuscript.

\section{Reviewer's comment:}

Page 8, line 11: what plasticity model was assumed for the tunnel lining? Add this in the manuscript.

\section{Authors' reply:}

An elastic-perfectly plastic material with a Mises yield surface was used to model the tunnel lining. The elastic modulus and the yield strength required for the calibration of the model were tuned, so as to correspond to the properties of the particular aluminium alloy. The following comment has been added in the revised manuscript:

Page: 8, lines: 16-19

"The tunnel lining was modelled using an elastic-perfectly plastic model with a Mises yield surface. The model, which is embedded in ABAQUS, is defined by the elastic modulus and the yield strength of the aluminium material [1]. The above parameters were tuned, so as to correspond to the properties of the particular aluminium alloy (Table 2)."

\section{Reviewer's comment:}

Page 8, line 13: It is only mentioned that "... a visco-elastic model or a visco-elasto-plastic model with Mohr-Coulomb..." were adopted for the soil: please provide details at this stage of the manuscript on the adopted models (what visco-elastic formulation?, what visco-elastoplastic?).

A first series of analysis were conducted using an elastic model with viscous damping (i.e. visco-elastic model). The soil stiffness, corresponding to the effective degraded stiffness, as well as the viscous damping, were back-calculated, by comparing the predictions of 1D equivalent linear soil response analyses with the recorded acceleration at the soil 'free-field'. The procedure is discussed in detail in section 3.2 of the revised manuscript. In the second series of analyses, the above model was combined with a Mohr-Coulomb model, so as to account for the yielding response of the soil. The sand equivalent properties (i.e. effective stiffness and viscous damping) were kept the same as in the visco-elastic analyses. The adopted soil constitutive models were selected as they are proposed in guidelines for the dynamic analysis of embedded structures and are commonly used by the tunnelling design 
practise due to their easy calibration and control. Actually, the visco-elasto-plastic model, which was implemented in the final series of analysis, has been recently used by Cilingir and Madabhushi for the simulation of similar dynamic centrifuge tests on model tunnels in dry sand, revealing reasonable comparisons between the recorded data and the numerical results (references [16-18] in the revised manuscript). To clarify this crucial aspect, the presentation of the implemented constitutive models has been revised in the manuscript, as follows:

Page: 8 , lines: 20-32

"The soil response under seismic shaking was simulated in two ways. In a first series of analyses, a visco-elastic model was implemented, introducing an effective sand shear modulus distribution and viscous damping (i.e. following the equivalent linear approximation method). The sand equivalent properties were back-calculated, as discussed in the following section. In the second series of analyses, a non-associated Mohr-Coulomb yield criterion was combined with the above visco-elastic model to account for the permanent deformations of the soil. To implement the latter model herein, the sand equivalent properties were set equal to those of the visco-elastic model. This elasto-plastic approach has been recently used by Cilingir and Madabhushi for the simulation of similar dynamic centrifuge tests on model tunnels in dry sand, revealing reasonable comparisons between the recorded data and the numerical results [16-18]. The above models were selected as they are proposed in guidelines for dynamic analysis of embedded structures (e.g. equivalent linear approximation in [20]) and are commonly used in tunnelling design practice due to their easy calibration and control."

\section{Reviewer's comment:}

Page 8, line 21: in those cases were a plasticity-based formulation was adopted for the soil, was it active during both the static and the dynamic stages? Does the soil experience yielding during the static stage? This is not clarified in the following part of the paper and should be detailed in this paragraph.

\section{Authors' reply:}

The plasticity model was active in both stages, so as to simulate in a simplified way the yielding of the soil occurred during the swing up of the centrifuge (i.e. increase of the gravity loads on the model). The elasto-plastic analyses revealed slight yielding of the soil during the static step, which was mainly concentrated at the middle sections of the slabs and the sidewalls of the tunnel. A relevant comment has been added in the revised manuscript:

Page: 12, lines: 1-3 (section 5.1)

"The visco-elasto-plastic analyses revealed slight yielding of the soil, which was concentrated near the middle sections of the slabs and the side-walls due to the inward deformations of these elements (deformations towards the cavity) during this step."

\section{Reviewer's comment:}

Page 8, line 23: it is stated that "... the loading history for the sand was accounted for": the state of the sand and its evolution during the setup of the model and the following centrifuge stages is a rather crucial ingredient which influences the observed dynamic behavior of the system. In fact, this is well highlighted by the Authors in the following parts of the manuscript, were they often attribute to this feature the discrepancies between the experimental results and the numerical ones. As such, it seems that the overall state evolution of the sand in only 
accounted for in a rather simplified way in the FE analyses: it is worth clarifying this limitation at this stage of the paper.

\section{Authors' reply:}

The accurate simulation of the evolution of the stress state of the sand in the centrifuge is a complicated task, especially in the case, where the soil properties are affected by the 'shadow and silo' effects (please see section 3.2). To make this clear, the following comment has been added in the revised manuscript:

Page: 9, lines: 2-4

"In a second step the dynamic analysis was performed by applying the shaking motions. The earthquake motions were applied in a row, in order to replicate the actual testing procedure and account for the loading history for the sand in a simplified way."

\section{Reviewer's comment:}

Page 9, line 9 and Page 10, line 18: when commenting Fig. 5(e) it should be declared that the overall picture is that of a disperse set of results, not terribly well back-fitted by the adopted fitting lines (instead of assessing that ".. are in relatively good agreement...").

\section{Authors' reply:}

The procedure proposed by Zeghal and Elgamal may provide only a rough estimation of the sand equivalent properties at the soil 'free-field' conditions. Actually, these properties (the presented shear moduli in this case) may be biased by the soil densification during shaking and the associated changes on the relative distances between the receivers during shaking. This may partly explain the relatively high dispersion of the 'experimental' values. Given this fact, the order of magnitude of the effective shear moduli is reproduced reasonably well by the numerical analyses. To account for this issue, the relevant paragraph has been revised as follows:

\section{Page: 11, lines:1-6}

"Fig. 5e compares the effective sand shear moduli computed by the procedure presented above with the values estimated by the acceleration records across the 'free-field' array, following the procedure outlined in Zeghal and Elgamal [54]. The latter may be biased to some extent by the changes on the relative distances between the receivers, associated with the densification of the soil during shaking. Accounting for the uncertainties related with the 'experimental' values, a fair comparison is observed."

\section{Reviewer's comment:}

Page 10, line 23: the sentence should be better reformulated, possibly providing a definition of flexibility (which should describe a relative soil-structure stiffness entity).

\section{Authors' reply:}

The particular paragraph has been revised in the manuscript, as follows:

Page: 11, lines: 7-11

"The effective sand stiffness described by Eq. 1 was encoded in the final numerical models of the soil-tunnel system through a FORTRAN user-subroutine, which correlates the stiffness with the mean effective stress at each soil element integration point. To this end, the effect of the tunnel on the mean effective stress and thus on the stiffness of the surrounding sand was explicitly accounted for. 


\section{Reviewer's comment:}

Page 10, line 26: it is not clear at all the selection criteria and the calibration procedure adopted to define the damping in the FE analyses. While this ingredient is well recognised in the literature as a key one in this class of analyses, no specific motivation for the selection of its target value of 15-17\% is given in the paper, nor is properly justified the selection of the two controlling frequencies of the adopted Rayleigh formulation. More in general: were the same target damping values assumed for all the simulations, irrespectively of the intensity and related shear strain amplitude induced by the different events? How does the free field response in centrifuge compare to the corresponding numerical one? Can the Author provide useful indication on a possible strategy to select the above parameters in a class-A prediction case (more useful and challenging)? Did the Authors modify the values of Rayleigh damping when switching from visco-elastic to visco-elasto-plastic analyses, to account for the plasticity-induced dissipation? All the above issues should be addressed in detail in a revised version of the paper.

\section{Authors' reply:}

Unfortunately, it was not possible to get a clear idea of the damping from the recorded accelerations, using the Zeghal and Elgamal procedure. Therefore the efficiency of the selected damping values was checked, based solely on the comparisons between the recorded and the computed inertial response of the soil-tunnel system (i.e. Fig. 8). The following procedure was followed for the calibration of damping: 'Target' damping was estimated for each shaking scenario, using the results of the one-dimensional equivalent linear soil response analyses (discussed in section 3.2 of the revised manuscript) for the finally adopted $G_{\max }$ and $G-\gamma-D$ curves. These results were then used to estimate 'average' values for the 'target' damping during each test (i.e. $17 \%$ for Test 1 and $15 \%$ for Test 2 ). The selection of average values for the 'target' damping facilitated the numerical analyses in which the seismic shakings were introduced in a row, given that the change of damping during the successive shakings was not possible. For the calibration of the Rayleigh parameters, the double frequency approach was implemented. The Rayleigh parameters were properly tuned for different 'important frequencies', so as to achieve good comparisons between the computed and recorded acceleration data. In this context, the calibration frequencies were set to $f_{l}=1$ $\mathrm{Hz}$ and $f_{2}=3 \mathrm{~Hz}$. Given that similar viscous damping was assumed in both visco-elastic and visco-elasto-plastic analyses, additional energy dissipation was introduced by the hysteretic soil response in the latter analyses.

The Authors agree that the selection of the Rayleigh parameters in a class-A prediction is useful and challenging. However, the proposal of a potential strategy for the selection of the above parameters in class-A predictions, is probably out of the scope of this paper, which aims to discuss important aspects of the response of rectangular tunnels in soft soils. The importance of proper calibration for the Rayleigh coefficients is discussed in detail by Kontoe et al. ([31] in the revised manuscript). In order to clarify these important aspects, the text was revised, as follows:

Page: 11, lines: 12-24

"In both visco-elastic and visco-elasto-plastic analyses, viscous damping was introduced in the form of the frequency dependent Rayleigh type. The one-dimensional equivalent linear soil response analyses for the finally adopted $G_{\text {max }}$ and $G-\gamma-D$ curves were used to evaluated 'target' damping for each shaking scenario. These results were then used to estimate 'average' values for the 'target' damping during each test (i.e. $17 \%$ for Test 1 and $15 \%$ for Test 2). The selection of average values for the 'target' damping facilitated the numerical 
analyses, given that the change of damping during the successive shakings was not possible. For the calibration of the Rayleigh parameters, the double frequency approach was implemented. The Rayleigh parameters were properly tuned for different 'important frequencies', so as to achieve good comparisons between the computed and recorded acceleration data. In this context, the calibration frequencies were set to $f_{1}=1 \mathrm{~Hz}$ and $f_{2}=3$ $\mathrm{Hz}$. The importance of proper calibration for the Rayleigh coefficients is discussed in Kontoe et al. [31]. It is noted that additional energy dissipation was introduced by the hysteretic soil response in the visco-elasto-plastic analyses."

\section{Reviewer's comment:}

Page 11, line 9: what do you mean with plastic strains? Are you referring to a specific component of the strain tensor or to one of its invariants? Please clarify.

\section{Authors' reply:}

The plastic strains discussed in the manuscript refer to the resultant of the plastic strain tensor. This is clarified in the revised version.

\section{Reviewer's comment:}

Page 12, line 2: in commenting Fig. 7, which only shows numerical results, it is concluded that "...were in good agreement with the results computed at the middle section of the soiltunnel model": it is not clear if the model mentioned here is the numerical or the centrifuge one? If it is the numerical, then clarify it; if it is the centrifuge, please delete the sentence as no data are yet provided to prove what stated.

\section{Authors' reply:}

The Reviewer's concern is relevant. The comment refers to the numerical model. To avoid misunderstandings, the text has been revised as follows:

Page: 12, lines: 21-22

"It is noteworthy that the 3D numerical model predicted almost identical internal forces at the corresponding locations of the middle section and the end sides of the tunnel."

\section{Reviewer's comment:}

Page 13, line 5: from Tables 4 and 5 it seems that the "reasonably good agreement" mentioned in the line is not that good. It is suggested to smooth the comment, highlighting the differences between observed and back-predicted data, possibly commenting on the very minor role played by the interface assumptions.

\section{Authors' reply:}

Following the Reviewer's suggestion, this section has been revised in the manuscript as follows:

\section{Page: 14, lines: 8-18}

"The numerical predictions are in a fair agreement with the recorded data (at least in the order of magnitude). The observed differences between the recorded and the computed data should be attributed to the several issues, including the relative simplification and calibration of the constitutive model, as well as uncertainties related to the calibration of the strain gauges. As mentioned above, the properties of the sand in the area around the tunnel were affected by the 'shadow and silo' effects during the model formation, making the calibration of the constitutive model cumbersome. Although the calibration of the strain gauges was done 
very carefully (i.e. [51]), there are always some unavoidable uncertainties related with the calibration of the above instruments, potentially causing some differences between the experimental and the numerical results."

\section{Reviewer's comment:}

Page 14, line 6: it appears that no major role is played by plasticity in the illustrated responses: the sentence justifies it stating that "... as equivalent sand properties were similar in both cases". Please clarify this point in light of what already discussed above on the same topic (page 10, line 26).

\section{Authors' reply:}

Indeed, the plasticity of the surrounding soil did not affect the inertial response of the soiltunnel system, significantly. Small differences were actually observed between visco-elastic and the visco-elasto-plastic analyses due to additional energy dissipation, induced by the hysteretic response of the soil in the latter case (i.e. elasto-plastic analyses). This is clarified in the revised manuscript:

Page: 15, lines: 16-19

"Small differences were actually observed between the predictions of the visco-elastic and visco-elasto-plastic analyses due to additional energy dissipation induced by the hysteretic response of the soil in the latter cases."

\section{Reviewer's comment:}

Page 21, line 4: differently from what concluded in the text, looking at Fig. 14 it appears that EC8 rigid wall solution does not compare well with the numerical results. The comment should be modified.

The comment is relevant. This state has been revised in the manuscript as follows:

Page: 22, lines: 8-12

"The earth pressures (dynamic part) computed following the prescriptions of the EC8 for non deformable rigid walls [11] are more conservative than the M-O approach. However, the results of both simplified approaches deviate considerably from the numerical predictions. Evidently, the simplified approaches are not capable to reproduce the actual earth pressures distribution pattern (e.g. concentrations of the earth pressures at the corners)." 


\section{Highlights:}

Dynamic centrifuge tests are performed on square tunnels in dry sand

The test cases are analyzed by means of full dynamic analysis

Results indicate a rocking deformation mode coupled with racking distortion

Interface conditions and soil yielding affect significantly the response

The effect of the lining rigidity on the response is highlighted 


\title{
On the Dynamic Response of Square Tunnels in Sand
}

\section{Grigorios Tsinidis, Kyriazis Pitilakis, Gopal Madabhushi}

\begin{abstract}
The paper investigates the seismic response of square tunnels in sand by means of dynamic centrifuge testing and numerical analysis. A series of dynamic centrifuge tests conducted at the University of Cambridge on a square aluminium model tunnel embedded in dry sand, are initially presented. The tests, which were designed in order to investigate the seismic response of flexible tunnels, are analyzed numerically be means of full dynamic analysis of the coupled soil-tunnel system, using different soil and soil-tunnel interface models. Numerical predictions are compared to the experimental data, in order to better understand the response mechanism and validate the numerical modelling. The validated numerical models are then used to investigate the effect of the lining rigidity on the soil-tunnel system dynamic response. The experimental and numerical results reported herein, indicate a non-negligible rocking deformation mode for the tunnels coupled with racking distortion during seismic shaking. The significant effects of the lining rigidity, soil-tunnel interface characteristics and soil yielding on the dynamic earth pressures and the shear stresses developed around the perimeter of the tunnel, as well as on the dynamic lining forces, are also reported and discussed.
\end{abstract}




\section{On the Dynamic Response of Square Tunnels in Sand}

3 Grigorios Tsinidis ${ }^{a}$, Kyriazis Pitilakis ${ }^{a}$, Gopal Madabhushi ${ }^{b}$

a Aristotle University of Thessaloniki, Department of Civil Engineering, Thessaloniki, Greece

${ }^{\mathrm{b}}$ University of Cambridge, Schofield Centre, Cambridge, UK

8 Corresponding Author: Grigorios Tsinidis, Aristotle University of Thessaloniki, Department 9 of Civil Engineering, GR-54124, PO BOX 424, Thessaloniki, Greece, e-mail:

10 gtsinidi@civil.auth.gr, tel: +30 2310994208

12 Abstract: The paper investigates the seismic response of square tunnels in sand by means of 13 dynamic centrifuge testing and numerical analysis. A series of dynamic centrifuge tests 14 conducted at the University of Cambridge on a square aluminium model tunnel embedded in 15 dry sand, are initially presented. The tests, which were designed in order to investigate the 16 seismic response of flexible tunnels, are analyzed numerically be means of full dynamic 17 analysis of the coupled soil-tunnel system, using different soil and soil-tunnel interface models.

18 Numerical predictions are compared to the experimental data, in order to better understand the 19 response mechanism and validate the numerical modelling. The validated numerical models are 20 then used to investigate the effect of the lining rigidity on the soil-tunnel system dynamic 21 response. The experimental and numerical results reported herein, indicate a non-negligible 22 rocking deformation mode for the tunnels coupled with racking distortion during seismic 23 shaking. The significant effects of the lining rigidity, soil-tunnel interface characteristics and 24 soil yielding on the dynamic earth pressures and the shear stresses developed around the 25 perimeter of the tunnel, as well as on the dynamic lining forces, are also reported and 26 discussed.

27 Keywords: Square tunnels, Dynamic centrifuge testing, Dynamic analysis, Interface 28 conditions, Tunnel rigidity

\section{Introduction}

31 Underground structures and tunnels behaved better than above ground structures during recent

32 strong earthquakes. However, several cases of severe damages to total collapse have been 33 reported in the literature (e.g. [27, 53]). Shallow embedded structures in soft soil were found 
more vulnerable to seismic shaking, while the vulnerability was generally increased in cases

2 where seismic design provisions were not encountered.

The seismic response of tunnels is quite distinct compared to that of above ground structures, as the kinematic loading induced by the surrounding ground prevails over inertial loads stemming from the oscillation of the tunnel itself. The response of underground structures and tunnels under ground shaking and earthquake induced ground failures has been a subject of intense research by a series of experimental $[2,12-14,16-18,33,46,50,52]$, numerical $[3-5,7,24-25,31-32,34,48]$ and analytical $[8-9,26]$ studies. However, several

9 crucial issues related to the seismic response of square tunnels are still under investigation, 10 including (i) the amplitude and distribution of the seismic earth pressures on the tunnel side11 walls, (ii) the soil dynamic shear stresses around the perimeter of the tunnel and (iii) the 12 complex deformation modes mobilized during shaking (e.g. rocking). In this context, 13 conventional design specifications are based primarily on simplified methods (e.g. [20, 23, 29, 14 40, 53]) the implementation of which may lead to substantial differences in the seismic design 15 of underground structures [42, 49].

16 This study aims to shed light on the above issues by means of dynamic centrifuge testing 17 and numerical analysis. In this context, a series of dynamic centrifuge tests, carried out on a 18 flexible square model tunnel embedded in dry sand, are initially presented. The tests, which 19 were performed at the geotechnical centrifuge facility of the University of Cambridge within 20 the TUNNELSEIS action of the SERIES research project, are analyzed numerically by means 21 of full dynamic analyses of the coupled soil-tunnel system. The numerical predictions are 22 compared to the recorded data, in order to better understand the response mechanism and 23 validate the numerical modeling. Additional analyses are performed, using the validated 24 numerical models, aiming to investigate the effect of the lining rigidity on the soil-tunnel system response. Through the presentation of the experimental and numerical results, the

26 significant effects of the soil-tunnel interface characteristics, lining rigidity and soil yielding on 27 the dynamic earth pressures, the soil dynamic shear stresses and the dynamic lining forces are 28 reported. Moreover, complex deformation modes are identified during shaking for this type of 29 structures.

\section{2. Dynamic centrifuge testing}

$31 \quad 2.1$ Centrifuge facility

32 The tests were conducted at the Turner beam centrifuge, under a centrifugal acceleration of 50 $33 \mathrm{~g}$ (scale factor $N=50)$. A large Equivalent Shear Beam (ESB) container was used to mount the 
1 models. The container, the inside dimensions of which are $673 \times 255 \times 427(\mathrm{~mm})$, consists of

2 aluminium dural frames, connected to each other through rubber layers, and is designed, so as

3 to share similar stiffness with the contained soil for the strains of interest [55]. The dynamic

4 input motion was imposed at the base of the container by a Stored Angular Momentum (SAM)

5 actuator, designed to apply sinusoidal or sine-sweep bedrock input motions [36]. A specially

6 designed on-board data acquisition system, called CDAQS (Centrifuge Data Acquisition

7 System) was used to digitize and save the recorded data. A second acquisition system that uses

8 the DASYLab software [19] was also implemented, allowing for higher sampling frequencies.

9 More details on dynamic centrifuge testing may be found in [35].

\section{$11 \quad 2.2$ Materials}

12 The soil models were made of dry Hostun HN31 sand, reconstituted at two different relative 13 densities $\left(D_{r} \approx 50 \%\right.$ for Test 1 and $\approx 90 \%$ for Test 2$)$. The physical properties of the sand are 14 tabulated in Table 1, whereas the particle size distribution is portrayed in Fig. 1a.

(a)

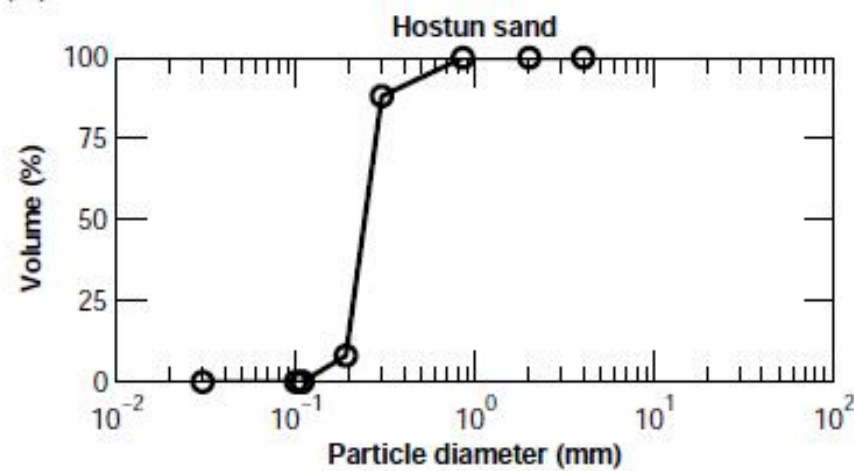

(b)

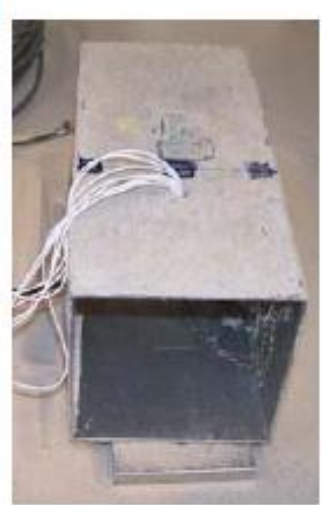

Fig. 1 (a) Particle size distribution of Hostun HN31 sand [41], (b) model tunnel

18 Table 1 Physical properties of Hostun HN31 sand [41]

\begin{tabular}{|c|c|c|c|c|c|c|}
\hline$\rho_{\mathrm{s}}\left(\mathrm{g} / \mathrm{cm}^{3}\right)$ & $\mathrm{e}_{\max }$ & $\mathrm{e}_{\min }$ & $\mathrm{d}_{10}(\mathrm{~mm})$ & $\mathrm{d}_{50}(\mathrm{~mm})$ & $\mathrm{d}_{60}(\mathrm{~mm})$ & $\varphi_{\text {crit }}\left({ }^{\circ}\right)$ \\
\hline 2.65 & 1.010 & 0.555 & 0.209 & 0.335 & 0.365 & 33 \\
\hline
\end{tabular}

A $100 \times 100 \times 210(\mathrm{~mm})$ square model tunnel was manufactured by an extruded section of 6063A aluminium alloy (Table 2) having a thickness of $2 \mathrm{~mm}$ (Fig. 1b). According to the scale factor $(N=50)$, the model corresponds to a $5 \times 5(\mathrm{~m})$ flexible square tunnel having an equivalent concrete lining thickness equal to $0.13 \mathrm{~m}$ (assuming the elastic modulus for equivalent concrete section $\mathrm{E}=30 \mathrm{GPa}$ ). This thickness is unrealistic in practice, as the design analysis for the static loads would have resulted in a much thicker lining. However, this selection was made in order to study the effect of high lining flexibility on the tunnel response, 
as well as to obtain clear measurements of the lining bending and axial strains. To simulate more realistically the soil-structure interface and investigate the effect of interface conditions on the response, Hostun sand was stuck on the external facade of the model tunnel in one of the test cases (Test 2), creating a rough surface.

Table 2 Model tunnel mechanical properties

\begin{tabular}{|c|c|c|c|}
\hline Unit weight, $\gamma\left(\mathrm{kN} / \mathrm{m}^{3}\right)$ & Elastic modulus, $\mathrm{E}(\mathrm{GPa})$ & Poisson ratio, $\mathrm{v}$ & Tensile strength, $\mathrm{f}_{\mathrm{bk}}(\mathrm{MPa})$ \\
\hline 2.7 & 69.5 & 0.33 & 220 \\
\hline
\end{tabular}

\section{$8 \quad 2.3$ Models preparation}

9 The sand pouring was performed using an automatic hopper system (Fig. 2a, [37, 56]). During

10 the pouring process the tunnel and all the embedded transducers were positioned in the sand 11 model (Fig. 2b-d). The burial depth of the tunnel ranged between $60 \mathrm{~mm}$ (Test 1) and $100 \mathrm{~mm}$ 12 (Test 2). The model tunnel was shorter than the container width, in order to avoid any 13 interaction between the tunnel and the box. Two polytetrafluoroethylene (PTFE) rectangular 14 plates were placed at each end of the tunnel to avoid the entry of sand into the tunnel. The 15 plates, which were marginally larger than the model tunnel $(110 \mathrm{~mm} \times 110 \mathrm{~mm} \times 10 \mathrm{~mm})$, 16 were connected to each other by a rod, which passed through the tunnel. After the preparation, 17 the models (Fig. 2e) were loaded onto the centrifuge and all the instruments were plugged into 18 the on-board acquisition systems (Fig. 2f).

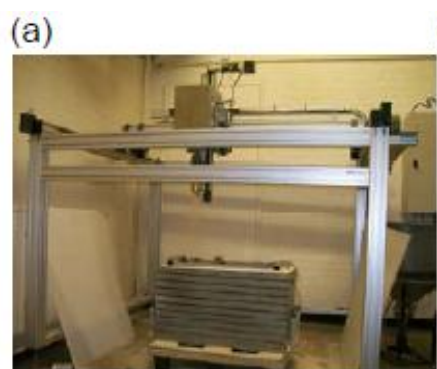

(d)

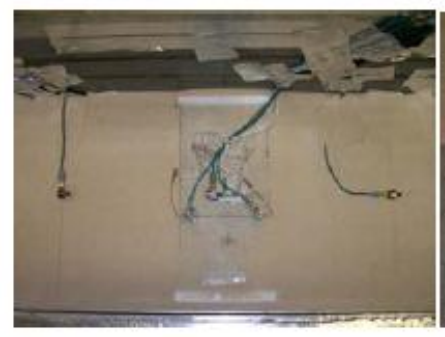

(b)

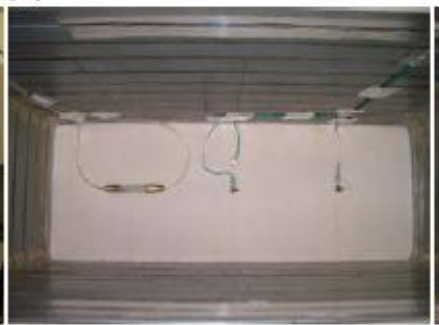

(e)

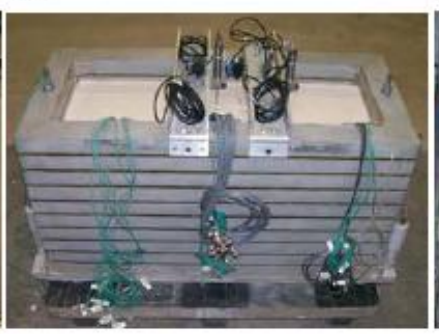

(c)

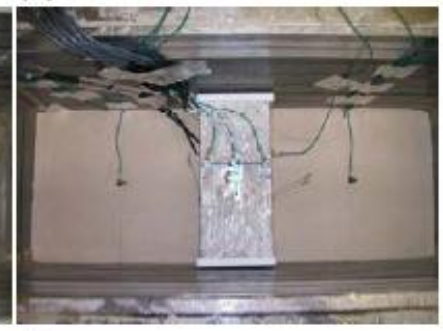

(f)

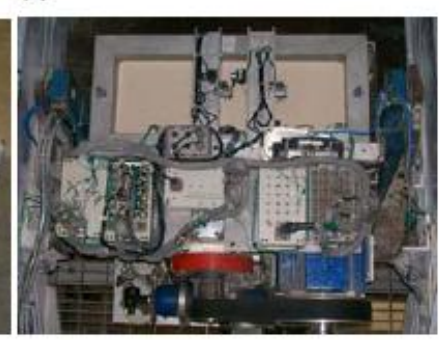

Fig. 2 Phases of the models construction (a) automatic hopper system and ESB container, (b-d)

21 placement of embedded transducers and of model tunnel, (e) completed model (f) loading of the ESB

22 container onto the centrifuge 


\section{$1 \quad 2.4$ Instrumentation scheme}

2 A dense instrumentation array was used to monitor the soil-tunnel response (Fig. 3). Miniature 3 piezoelectric accelerometers (A or $\mathrm{AH}$ in Fig. 3) were introduced in vertical arrays within the

4 soil and attached on the tunnel section and on the container to record the horizontal and vertical

5 acceleration. The soil surface settlements were recorded in two locations using linear variable

6 differential transformers (LVDTs in Fig. 3). Two string potentiometers (POTs in Fig. 3) were

7 attached to the tunnel to monitor its vertical displacement and possible rocking during shaking.

8 The above instruments were connected to the tunnel through small cables that were attached to

9 the side-walls using small screws. Two miniature total earth pressure cells (PCs in Fig. 3) were

10 attached to the left side-wall of the tunnel, allowing the record of the soil earth pressures on the

11 wall. Strain gauges were attached to the inner and outer faces of the model tunnel to record the

12 lining bending moment and axial force at crucial lining sections (SGs in Fig. 3). Unfortunately,

13 the gauges did not record during the first test (Test 1) due to a wiring problem, while they

14 worked properly during the second test (Test 2). All the instruments were properly calibrated 15 before and checked after testing. Regarding the strain gauges, their calibration was made 16 carefully for simple static loading patterns using the procedure outlined in Tsinidis et al. [51].

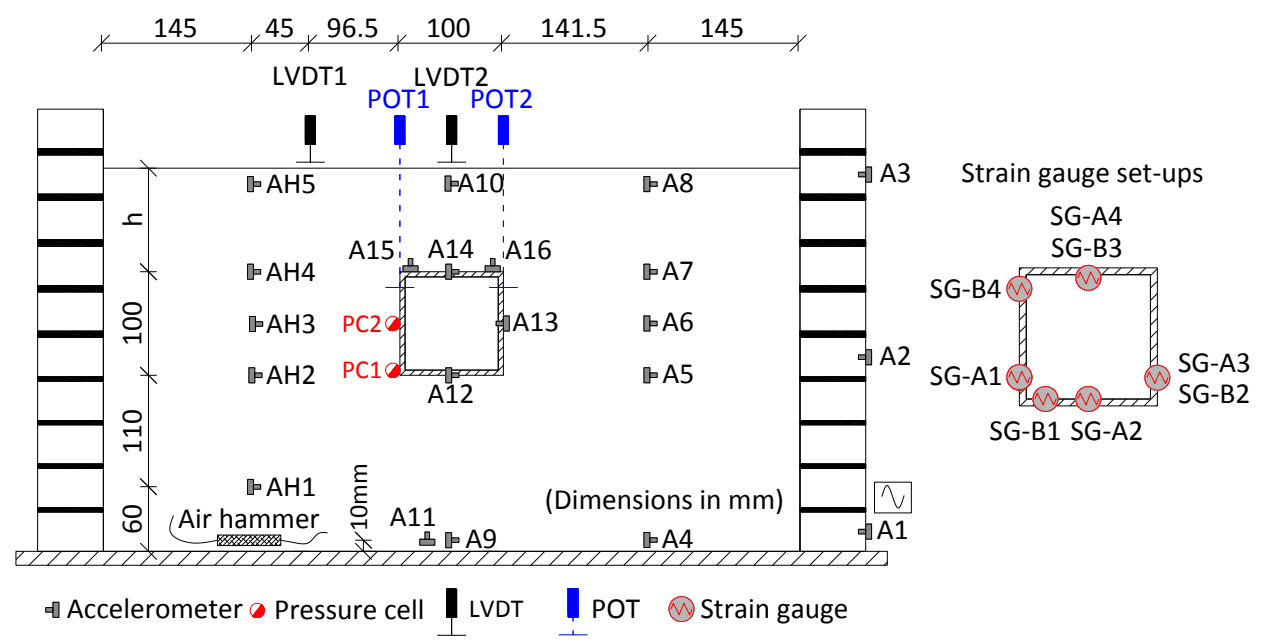

Fig. 3 Typical models layout $(\mathrm{h}=60 \mathrm{~mm}$ for Test $1,100 \mathrm{~mm}$ for Test 2$)$

\section{$20 \quad 2.5$ Testing program}

21 The models were subjected to a series of pseudo-harmonic shaking motions. During each test,

22 the centrifuge was spun up in steps until $50 \mathrm{~g}$. Subsequently, the shaking motions were fired in

23 a row, leaving some time between them to acquire the data. Table 3 tabulates the sequence and

24 characteristics of the shaking motions during each test. The data was recorded at a sampling

25 frequency of $4 \mathrm{~Hz}$ during the centrifuge swing up and at $4 \mathrm{kHz}$ during shaking. 
Air hammer tests were conducted during testing, in order to evaluate the soil shear wave

2 velocity gradient away from the tunnel, following the procedure outlined in Ghosh and

3 Madabhushi [21]. A small air hammer was introduced close to the base of the soil layer, while

4 a set of accelerometers were placed above it (AH in Fig. 3), allowing a record of the arrival

5 times of the waves emanating from the air hammer. To ensure that the arrival times were

6 adequately recorded, the accelerometers along this array were attached on an acquisition

7 system that used the DASYLab software [19], allowing for a higher sampling frequency of 50

$8 \mathrm{kHz}$.

10 Table 3 Centrifuge tests details (bracketed values in prototype scale)

\begin{tabular}{|c|c|c|c|c|c|c|c|c|c|}
\hline $\begin{array}{c}\text { Test } \\
\#\end{array}$ & $\begin{array}{c}\text { Tunnel external } \\
\text { rugosity }\end{array}$ & $\begin{array}{l}\text { Burial } \\
\text { depth } \\
\text { (mm) }\end{array}$ & $\begin{array}{c}\mathrm{D}_{\mathrm{r}} \\
(\%)\end{array}$ & $\begin{array}{l}\text { EQ } \\
\text { ID }\end{array}$ & Flight & $\begin{array}{l}\text { Input } \\
\text { type* }\end{array}$ & $\begin{array}{l}\text { Frequency } \\
(\mathrm{Hz})\end{array}$ & $\begin{array}{l}\text { Amplitude } \\
\text { (g) }\end{array}$ & $\begin{array}{c}\text { Nominal } \\
\text { duration } \\
\text { (s) }\end{array}$ \\
\hline \multirow{4}{*}{$\begin{array}{c}\text { Test } \\
1\end{array}$} & \multirow{4}{*}{$\begin{array}{c}\text { smooth external } \\
\text { face }\end{array}$} & \multirow{4}{*}{60} & \multirow{4}{*}{51} & EQ1 & 1 & $\mathrm{PH}$ & $60(1.2)$ & $10.5(0.21)$ & $0.4(20)$ \\
\hline & & & & EQ2 & 1 & $\mathrm{PH}$ & $60(1.2)$ & $12.9(0.26)$ & $0.4(20)$ \\
\hline & & & & EQ3 & 1 & $\mathrm{PH}$ & $60(1.2)$ & $15.7(0.31)$ & $0.4(20)$ \\
\hline & & & & EQ4 & 1 & $\mathrm{PH}$ & $60(1.2)$ & $18.32(0.37)$ & $0.4(20)$ \\
\hline \multirow{8}{*}{$\begin{array}{c}\text { Test } \\
2\end{array}$} & \multirow{8}{*}{$\begin{array}{c}\text { rough external } \\
\text { face }\end{array}$} & \multirow{8}{*}{100} & \multirow{8}{*}{89} & EQ1 & 1 & $\mathrm{PH}$ & $30(0.6)$ & $1.0(0.02)$ & $0.4(20)$ \\
\hline & & & & EQ2 & 1 & $\mathrm{PH}$ & $45(0.9)$ & $4.0(0.08)$ & $0.4(20)$ \\
\hline & & & & EQ3 & 1 & $\mathrm{PH}$ & $50(1.0)$ & $6.5(0.13)$ & $0.4(20)$ \\
\hline & & & & EQ4 & 1 & $\mathrm{PH}$ & $50(1.0)$ & $12.0(0.24)$ & $0.4(20)$ \\
\hline & & & & EQ5 & 1 & SS & $60(1.2)$ & $12.0(0.24)$ & $3.0(150)$ \\
\hline & & & & EQ6 & 2 & $\mathrm{PH}$ & $50(1.0)$ & $5.8(0.116)$ & $0.4(20)$ \\
\hline & & & & EQ7 & 2 & $\mathrm{PH}$ & $50(1.0)$ & $6.0(0.12)$ & $0.6(30)$ \\
\hline & & & & EQ8 & 2 & $\mathrm{PH}$ & $50(1.0)$ & $11.0(0.22)$ & $0.5(25)$ \\
\hline
\end{tabular}

*PH: Pseudo-Harmonic shake, SS: Sine sweep shake

\section{$13 \quad 2.6$ Interpretation of the experimental data}

14 To interpret the experimental results, the data was windowed, neglecting the parts of the 15 signals before and after the main shake duration, while a filtering procedure was conducted in

16 the frequency domain. In particular, acceleration-time histories were filtered at 10 to $400 \mathrm{~Hz}$

17 (in model scale) using a band pass eighth-order Butterworth type filter. All the other data were

18 filtered using a low-pass eighth-order Butterworth filter at $400 \mathrm{~Hz}$. Processed acceleration time

19 histories were used to evaluate the soil equivalent shear moduli, following Zeghal and Elgamal

20 [54] and Brennan [10]. More details about the experimental program may be found in [47].

\section{3. Numerical analysis}

\section{$22 \quad 3.1$ Description of the numerical models}

23 The tests were analyzed at prototype scale by means of full dynamic time history analyses of 24 the soil-tunnel systems, using the finite element code ABAQUS [1]. Appropriate scaling laws 
were used to convert the recorded quantities from model to prototype scale [44]. The numerical

2 analyses were carried out assuming plane strain conditions (Fig. 4a). To verify the above assumption and investigate possible 3D effects, additional analyses were conducted for one test case (Test 2), implementing a three-dimensional numerical model of the soil-tunnel system (Fig. 4b).

For the two-dimensional (2D) analyses, the soil was meshed with quadratic plane strain elements, while the tunnel was simulated with beam elements. For the three-dimensional (3D) analyses, 3D solid elements and shell elements were used for the soil and the tunnel lining,

9 respectively. The element size in both cases was selected in a way that ensured the efficient 10 reproduction of the waveforms of the whole frequency range under study (i.e. $0.2-8 \mathrm{~Hz}$ in 11 prototype scale). In particular, the maximum size of the elements was set equal to $0.6 \mathrm{~m}$ and $121.0 \mathrm{~m}$ for the $2 \mathrm{D}$ and the $3 \mathrm{D}$ analyses, respectively, while the mesh was generally finer near the 13 tunnel. More refined models, used during preliminary sensitivity analyses had a negligible 14 effect on the computed results, increasing significantly the computational cost.
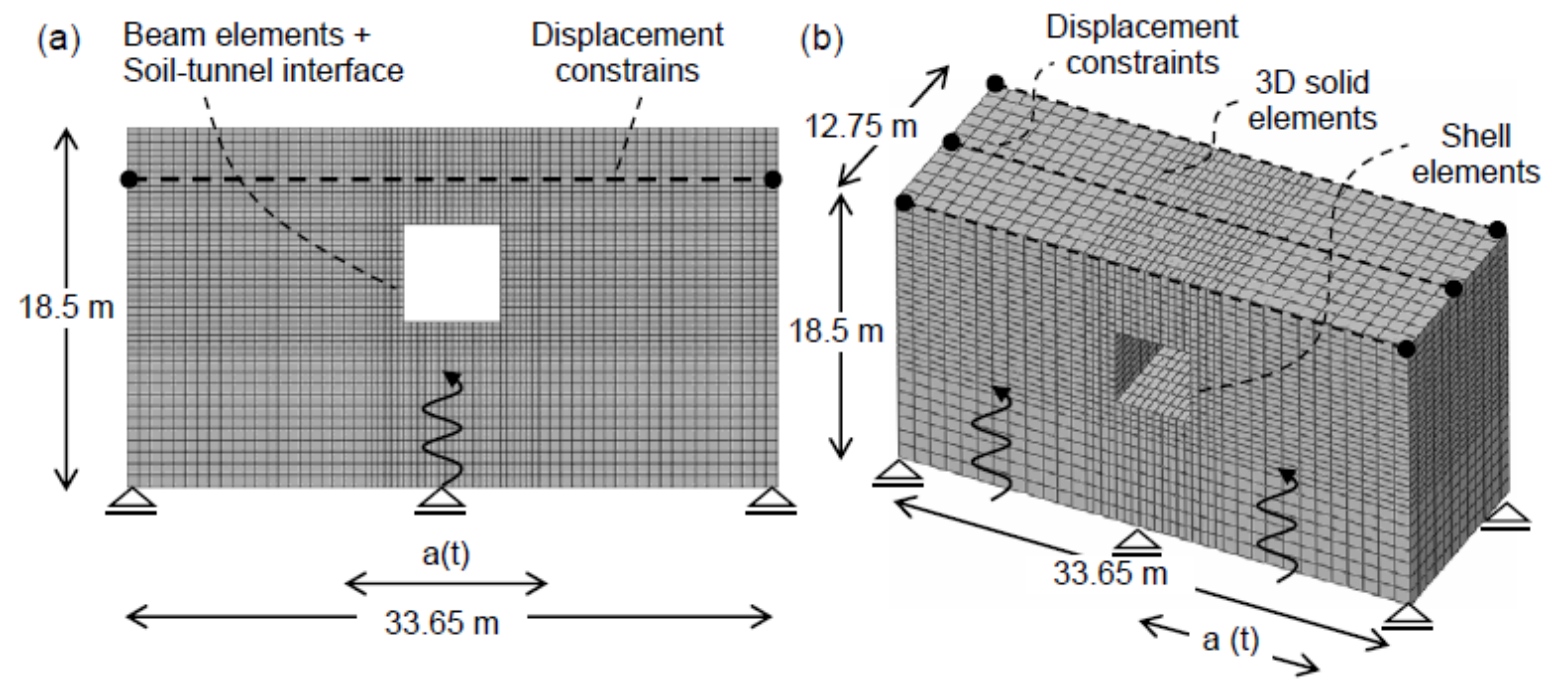

16 Fig. 4 (a) 2D and (b) 3D numerical model of Test 2

The base boundary of the 2D model was simulated as rigid bedrock (shaking table), whereas

19 tie constraints were introduced at the side boundaries kinematic, forcing the opposite vertical 20 sides to move simultaneously. Similar boundary conditions were adopted at the base and the 21 end-walls of the 3D model. In addition, the front and back sides of the model were constrained properly, in order to simulate the support of the container walls on the sand.

23 The soil-tunnel interface was modelled using a finite sliding hard contact algorithm, which 24 allows for separation between the soil and tunnel elements [1]. The tangential behavior of the interface was modelled using the penalty friction formulation, introducing the Coulomb 
1 friction model [1]. The effect of the interface friction on the soil-tunnel system response was

2 parametrically investigated by applying different Coulomb friction coefficients $\mu$, namely $\mu=0$

3 for the full-slip interface conditions and 0.4 and 0.8 for slip interface conditions. The latter

4 values correspond to soil-tunnel interface friction angles equal to $21.8^{\circ}$ and $38.6^{\circ}$, respectively

5 and constitute a lower and an upper limit for the sand-aluminium interface friction, according

6 to Kishida and Uesugi [30]. In a final series of analyses, the soil and the tunnel elements were

7 fully bonded, assuming no-slip conditions and precluding separation. The 3D analysis was

8 performed only for full bonding between the soil and tunnel elements, as this case provided

9 results closer to the recorded data for the specific test case. The better performance of full

10 bonding conditions in the particular test case is discussed in the ensuing.

11 Additional analyses were performed using the validated 2D numerical model configurations,

12 aiming to investigate the effect of lining rigidity on the dynamic response of the system. The

13 lining thickness was increased in these cases to $10 \mathrm{~mm}$ in model scale, corresponding to an

14 equivalent concrete lining thickness equal to $0.70 \mathrm{~m}$ in prototype scale (i.e. assuming the

15 elastic modulus for equivalent concrete section $\mathrm{E}=30 \mathrm{GPa}$ ).

16 The tunnel lining was modelled using an elastic-perfectly plastic model with a Mises yield

17 surface. The model, which is embedded in ABAQUS, is defined by the elastic modulus and the

18 yield strength of the aluminium material [1]. The above parameters were tuned, so as to

19 correspond to the properties of the particular aluminium alloy (Table 2).

20 The soil response under seismic shaking was simulated in two ways. In a first series of 21 analyses, a visco-elastic model was implemented, introducing an effective sand shear modulus 22 distribution and viscous damping (i.e. following the equivalent linear approximation method).

23 The sand equivalent properties were back-calculated, as discussed in the following section. In

24 the second series of analyses, a non-associated Mohr-Coulomb yield criterion was combined

25 with the above visco-elastic model to account for the permanent deformations of the soil. To

26 implement the latter model herein, the sand equivalent properties were set equal to those of the

27 visco-elastic model. This elasto-plastic approach has been recently used by Cilingir and

28 Madabhushi for the simulation of similar dynamic centrifuge tests on model tunnels in dry

29 sand, revealing reasonable comparisons between the recorded data and the numerical results

30 [16-18]. The above models were selected as they are proposed in guidelines for dynamic

31 analysis of embedded structures (e.g. equivalent linear approximation in [20]) and are

32 commonly used in tunnelling design practice due to their easy calibration and control.

33 The input motion was introduced at the base of the numerical models, as acceleration time

34 histories, referring to the motion recorded by the reference accelerometer (A1, Fig. 3). The 
analyses were performed in two steps. Initially, the gravity loads were introduced, within a

2 static step. In a second step the dynamic analysis was performed by applying the shaking

3 motions. The earthquake motions were applied in a row, in order to replicate the actual testing

4 procedure and account for the loading history for the sand in a simplified way. An automatic

5 scheme was used for the time incrementation during the dynamic analysis [1].

\section{$7 \quad 3.2$ Sand properties}

8 The small-strain shear modulus of the sand $G_{\max }$ was described according to Hardin and 9 Drenvich [22]. The model fits reasonably well with the air hammer test results, as well as with 10 results of laboratory resonant column and cyclic triaxial tests that were conducted on the 11 specific sand fraction (Fig. 5a, 5c). It is worth noticing that these results refer to the 'free-field' 12 conditions away from the model tunnel. The exact properties of sand in the area close to the 13 tunnel can not be well known, as they are affected by the 'shadow and silo' effects. These 14 effects, which are associated with the model construction method (i.e. sand pouring from a 15 height), affect the density of the sand in the adjacent zone of the tunnel (i.e. reduction of the 16 sand density), thus altering the mechanical properties of the sand at this area compared to the 17 'free-field' conditions.

18 The sand equivalent properties were estimated through a trial and error procedure, by 19 comparing the predictions of one-dimensional (1D) soil response analysis of the soil deposits 20 with the horizontal acceleration recorded at the soil 'free-field' (i.e. accelerometers A4 to A8 in 21 Fig. 3) during different shakings. The one-dimensional (1D) analyses were conducted in the 22 frequency domain with the numerical code EERA [6], using different sets of $G-\gamma-D$ curves for 23 cohesionless soils (e.g. [28, 41, 45]). The adopted $G-\gamma-D$ curves were those that resulted in the 24 best fitting of the numerical predictions with the experimental results (Ishibashi and Zhang 25 [28], for sand and small confining pressure). Comparisons of the adopted $G-\gamma-D$ curves with 26 empirical ones [45], as well as laboratory results from resonant column and cyclic triaxial tests 27 for the specific sand fraction [41] are provided in Fig. 5b and 5d for the examined test cases. 28 The adopted numerical curves compare reasonably well with the laboratory test results over a 29 wide range of strain amplitudes. One-dimensional equivalent linear soil response analyses for 30 the finally selected $G_{\max }$ and $G-\gamma-D$ curves revealed that a reduced Hardin and Drenvich 31 distribution adequately reproduced the degraded sand shear modulus during shaking. To this 32 end, the following expression was used for the description of the degraded strain shear 33 modulus: 


$$
G=\left[\alpha \times 100 \times(3-e)^{2} \times\left(\sigma^{\prime}\right)^{0.5}\right] /(1+e)
$$

2 where $e$ is the void ratio, $\sigma^{\prime}$ is the mean effective stress (in $\mathrm{MPa}$ ) and $G$ is the shear modulus

3 (in $\mathrm{MPa}$ ) and $\alpha$ is the reduction value for each shake, ranging between $0.28-0.4$.

(a)

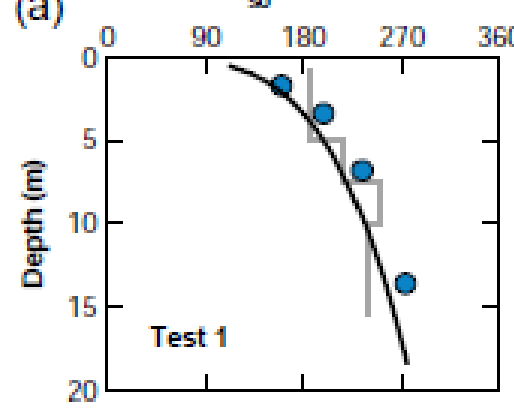

(c) $\quad \mathrm{v}_{\mathrm{so}}(\mathrm{m} / \mathrm{s})$

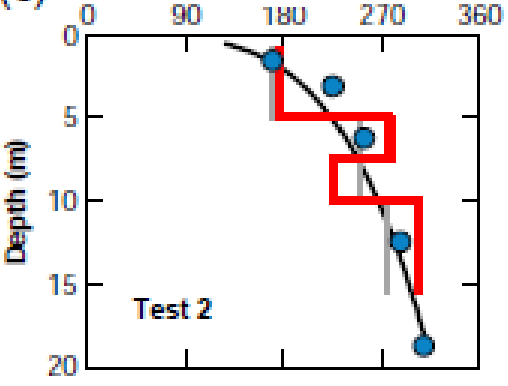

Hardin and Drenvic (1972) AH, Flight 1

AH, Flight 3

Pistolas et al. (2014) (b)
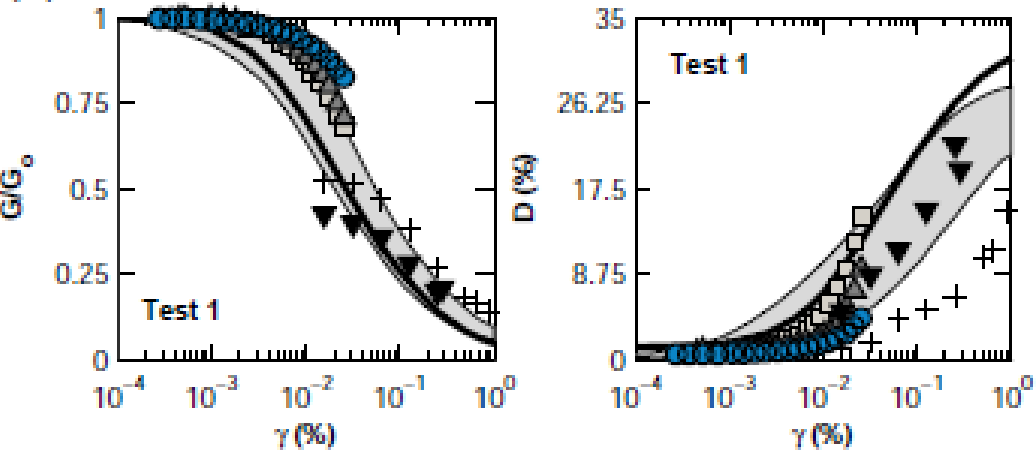

(d)

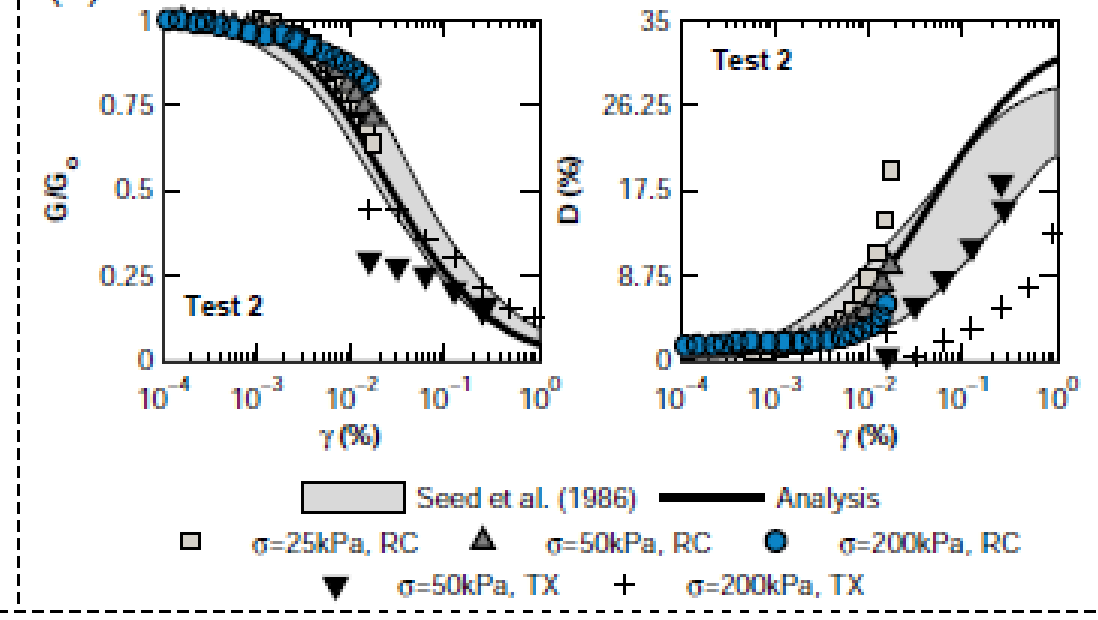

(e) EQ1 - Test 1 (0.21g)

EQ4 - Test $1(0.37 \mathrm{~g})$ $\mathrm{G}(\mathrm{MPa})$
EQ2 - Test 2 (0.08g) $\mathrm{G}(\mathrm{MPa})$
EQ5 - Test $2(0.24 \mathrm{~g})$ $\mathrm{G}(\mathrm{MPa})$
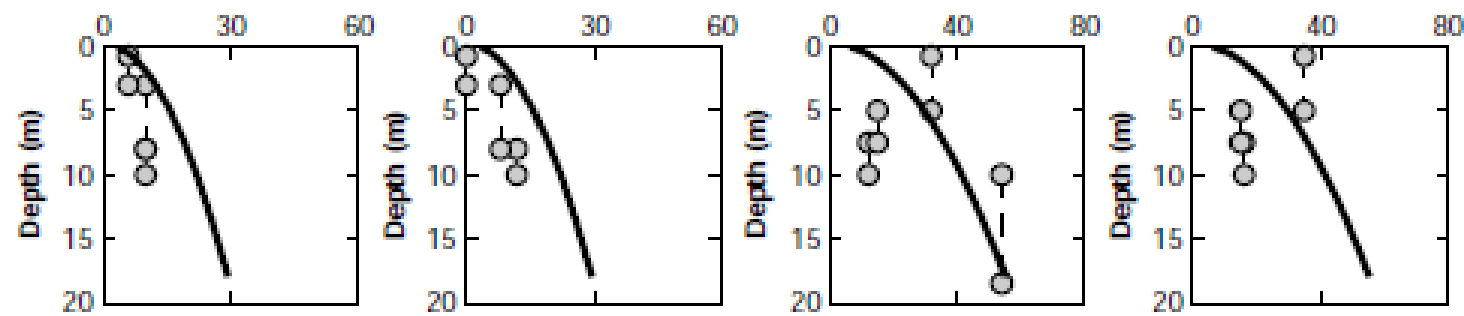

Numerical analysis

Fig. 5 Calibration of sand models, (a, c) Small-strain shear wave velocity gradients estimated from air hammer tests (AH) and resonant column tests (RC) [41] compared to Hardin and Drenvich empirical formulation [22], (b, d) adopted $G-\gamma-D$ curves compared to resonant column test results (RC) [41], cyclic triaxial test results (TX) [41] and empirical proposals [45], (e) comparisons of effective soil shear

9 moduli adopted in the numerical analysis with the values revealed by the recorded data, following [40, 
Fig. 5e compares the effective sand shear moduli computed by the procedure presented above with the values estimated by the acceleration records across the 'free-field' array, following the procedure outlined in Zeghal and Elgamal [54]. The latter may be biased to some extent by the changes on the relative distances between the receivers, associated with the densification of the soil during shaking. Accounting for the uncertainties related with the 'experimental' values, a fair comparison is observed.

The effective sand stiffness described by Eq. 1 was encoded in the final numerical models

8 of the soil-tunnel system through a FORTRAN user-subroutine, which correlates the stiffness

9 with the mean effective stress at each soil element integration point. To this end, the effect of

10 the tunnel on the mean effective stress and thus on the stiffness of the surrounding sand was

11 explicitly accounted for.

12 In both visco-elastic and visco-elasto-plastic analyses, viscous damping was introduced in

13 the form of the frequency dependent Rayleigh type. The one-dimensional equivalent linear soil 14 response analyses for the finally adopted $G_{\max }$ and $G-\gamma-D$ curves were used to evaluated 15 'target' damping for each shaking scenario. These results were then used to estimate 'average' 16 values for the 'target' damping during each test (i.e. $17 \%$ for Test 1 and $15 \%$ for Test 2). The 17 selection of average values for the 'target' damping facilitated the numerical analyses, given 18 that the change of damping during the successive shakings was not possible. For the calibration 19 of the Rayleigh parameters, the double frequency approach was implemented. The Rayleigh 20 parameters were properly tuned for different 'important frequencies', so as to achieve good 21 comparisons between the computed and recorded acceleration data. In this context, the 22 calibration frequencies were set to $f_{1}=1 \mathrm{~Hz}$ and $f_{2}=3 \mathrm{~Hz}$. The importance of proper calibration 23 for the Rayleigh coefficients is discussed in Kontoe et al. [31]. It is noted that additional energy 24 dissipation was introduced by the hysteretic soil response in the visco-elasto-plastic analyses.

25 The strength parameters of the sand were adopted, following Schanz and Vermeer [43]. In 26 particular, friction angle $\varphi$ was set equal to $33^{\circ}$ (critical friction angle for specific sand 27 fraction), while the dilatancy angle $\psi$ was assumed equal to $3^{\circ}$. The above strength parameters 28 were selected, so as to be compatible with the looser conditions of the sand area around the 29 tunnel, which are crucial for the tunnel response. As mentioned in section 3.2, the densification 30 level of this area is reduced compared to the 'free-field' due to the 'shadow and silo' effects, 31 associated with the formation of the sand model. A slight cohesion ( $c=1 \mathrm{kPa})$ was introduced 32 to avoid numerical instabilities. 


\section{4. Two-dimensional versus three-dimensional analysis}

2 The numerical predictions of the 3D analysis were compared with the results of the $2 \mathrm{D}$ 3 analyses, in order to validate the assumption of plain strain conditions and investigate potential 4 3D effects. Fig. 6 presents distributions of the soil plastic strains (i.e. resultant of the plastic 5 strain tensor) computed at the completion of the shaking motions EQ2 (0.08 g) and EQ4 (0.24

$6 \mathrm{~g}$ ) (Table 3). The distributions are plotted on deformed shapes of the numerical model. The 7 computed soil plastic strains, as well as the deformed shapes of the numerical model, reveal a uniform response of the soil-tunnel system along the longitudinal axis of the tunnel.

(a)

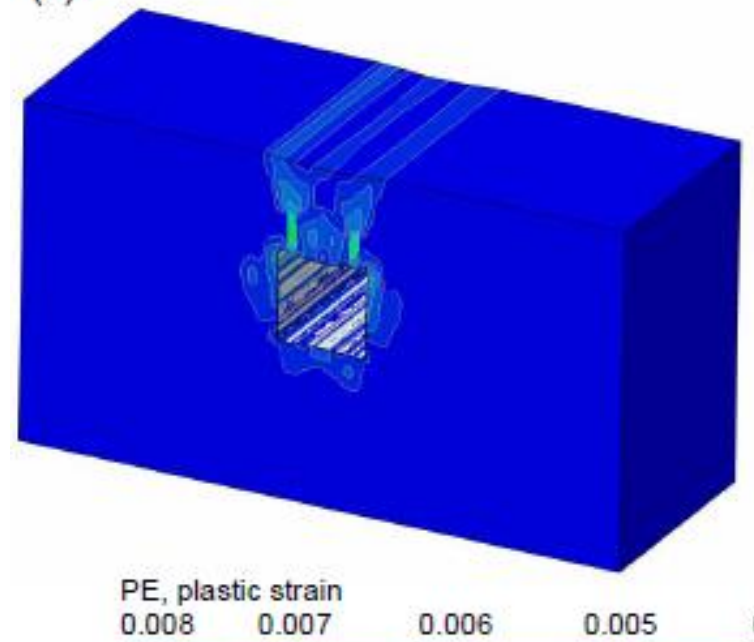

(b)

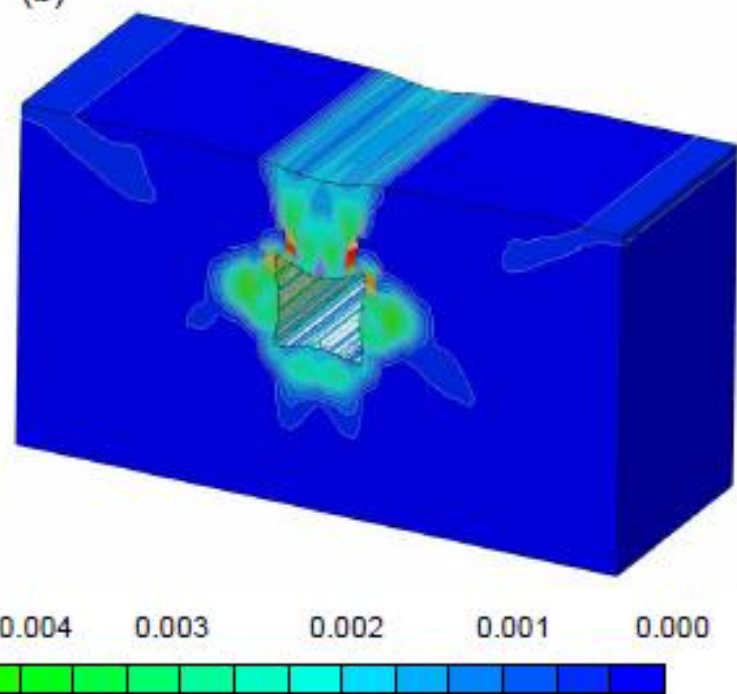

10 Fig. 6 Distributions of the soil plastic strains plotted on deformed shapes of the 3D numerical model (a) EQ2 (0.08g), (b) EQ4 (0.24g) (deformation scale × 30)

Figure 7 compares numerical predictions of the 2D and 3D numerical models, referring to shaking motion EQ4 (Table 3). The presented results of the 3D analysis refer to the actual positions of the recording devices in the test. The comparisons are made in terms of dynamic settlements of the soil surface (Fig. 7a), acceleration time histories (Fig. 7b), dynamic earth pressures time histories on left side-wall (Fig. 7c), dynamic bending moment time histories (Fig. 7d) and dynamic axial force time histories (Fig. 7e). The numerical predictions of the 3D analysis are in very good agreement with the results of the 2D analysis, verifying the validity of the plane strain behavior of the soil-tunnel system, adopted in 2D analysis. It is noteworthy that the 3D numerical model predicted almost identical internal forces at the corresponding locations of the middle section and the end sides of the tunnel. 

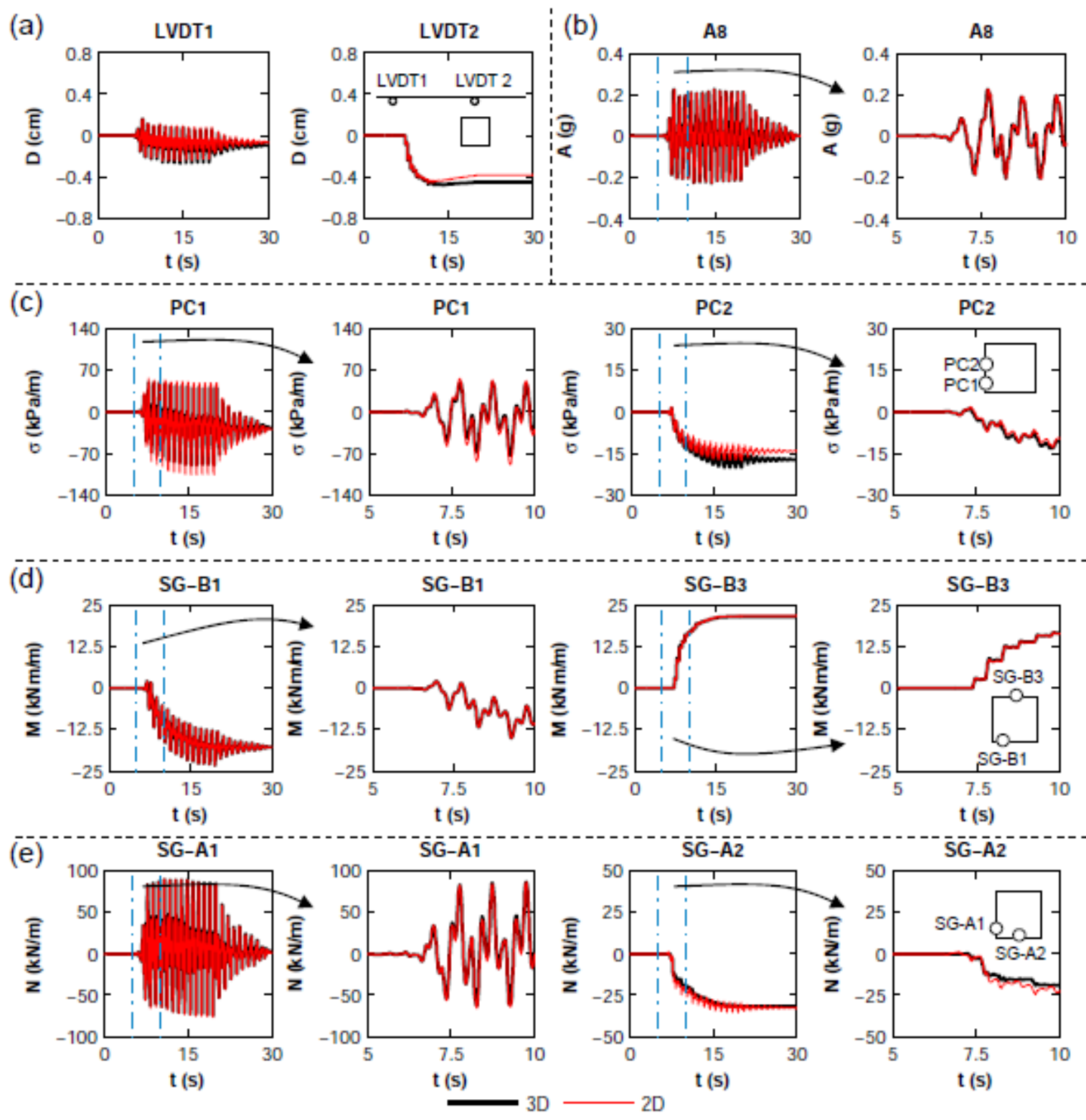

2 Fig. 7 Comparisons of 2D and 3D analysis predictions, (a) soil surface settlements (b) acceleration time

3 histories at the surface of the 'free-field' accelerometers vertical array, (c) dynamic earth pressures time

4 histories, (d) dynamic bending moment time histories, (e) dynamic axial force time histories

\section{5. Discussion of the results}

7 The following sections present and discuss recorded data and numerical results of the 2D

8 analyses, highlighting crucial aspects of the dynamic response of the soil-tunnel system. The

9 results refer to prototype scale, if not stated otherwise.

\section{$11 \quad 5.1$ Static response}

12 The internal forces of the tunnel increased during the swing up of the centrifuge due to the

13 increase of the model self weight. A similar response was reported by the numerical analyses 
1 during the static step. The visco-elasto-plastic analyses revealed slight yielding of the soil,

2 which was concentrated near the middle sections of the slabs and the side-walls due to the inward deformations of these elements (deformations towards the cavity) during this step.

Table 4 summarizes the static bending moments computed at the locations of the bending moment strain gauges by the visco-elasto-plastic analyses for different interface conditions.

6 The numerical results, referring to Test 2, are compared with the recorded data of both flights

7 of the centrifuge. The soil-tunnel interface characteristics affect the soil stress state around the 8 tunnel, thus diversifying slightly the bending moments computed by each analysis. The 9 numerical predictions are in a fair agreement with the recorded data (at least in the order of 10 magnitude). The observed differences between the recorded and the computed data should be 11 attributed to the several issues, including the relative simplification and calibration of the 12 constitutive model, as well as uncertainties related to the calibration of the strain gauges. As 13 mentioned above, the properties of the sand in the area around the tunnel were affected by the 14 'shadow and silo' effects during the model formation, making the calibration of the 15 constitutive model cumbersome. Although the calibration of the strain gauges was done very 16 carefully (i.e. [51]), there are always some unavoidable uncertainties related with the 17 calibration of the above instruments, potentially causing some differences between the experimental and the numerical results.

Table 4 Static bending moments recorded and computed by the visco-elasto-plastic analyses at the

21 strain gauges locations (Test 2)

\begin{tabular}{|c|c|c|c|c|c|}
\hline \multicolumn{5}{|c|}{ Bending moment $(\mathrm{kNm} / \mathrm{m})$} & \multirow{3}{*}{ SG-B3 } \\
\hline & SG-B1 & SG-B2 & SG-B3* & SG-B4 & \\
\hline Test - flight 1 & -27.50 & -37.00 & - & -37.50 & \\
\hline Test - flight 2 & -28.50 & -39.00 & - & -42.00 & G-E \\
\hline Analysis $-\mu=0$ & -20.75 & -19.65 & 42.48 & -35.60 & \\
\hline Analysis $-\mu=0.4$ & -20.13 & -19.75 & 38.88 & -32.73 & SG-B1 \\
\hline Analysis $-\mu=0.8$ & -20.35 & -19.63 & 38.15 & -32.58 & \\
\hline Analysis - No-slip conditions & -20.90 & -16.23 & 37.05 & -32.15 & \\
\hline
\end{tabular}

* malfunctioned during testing

23 Table 5 Static axial forces recorded and computed by the visco-elasto-plastic analyses at the strain

24 gauges locations (Test 2)

\begin{tabular}{|c|c|c|c|c|c|}
\hline \multicolumn{5}{|c|}{ Axial force $(\mathrm{kN} / \mathrm{m})$} & \multirow{8}{*}{ SG-A4 } \\
\hline & SG-A1 & SG-A2 & SG-A3 & SG-A4 & \\
\hline Test - flight 1 & -320.50 & -113.50 & -150.00 & -67.50 & \\
\hline Test - flight 2 & -322.00 & -123.00 & -177.50 & -77.00 & \\
\hline Analysis $-\mu=0$ & -217.00 & -103.00 & -217.00 & -95.50 & \\
\hline Analysis $-\mu=0.4$ & -231.50 & -102.50 & -231.50 & -77.50 & \\
\hline Analysis $-\mu=0.8$ & -240.00 & -100.00 & -239.50 & -73.50 & \\
\hline Analysis - No-slip conditions & -289.50 & -112.00 & -289.50 & -74.50 & \\
\hline
\end{tabular}


Similar conclusions are drawn by comparing the static axial forces recorded and the

2 computed at the axial strain gauges locations (Table 5). The large deviation between the 3 recorded axial forces in the symmetrical positions SG-A1 and SG-A3 is related to the large

4 difference observed in the calibration factors of the particular strain gauges [51].

\section{$6 \quad 5.2$ Dynamic response}

\section{$7 \quad$ 5.2.1 Horizontal acceleration}

8 Figure 8a compares time windows of acceleration time histories recorded and computed at the 9 middle section of the tunnel's right side-wall, referring to both test cases. In Figure $8 \mathrm{~b}$ 10 representative comparisons of horizontal acceleration amplification computed and recorded 11 along vertical arrays of accelerometers are depicted, referring to the first flight of Test 2. Solid 12 lines refer to the predictions of the visco-elasto-plastic analysis, whereas markers stand for the 13 recorded data. The numerical predictions are in good agreement with the records both in terms 14 of amplitude and frequency content. Some minor deviations are attributed to the inevitable 15 differences between the soil mechanical properties (stiffness and damping) adopted in the 16 analyses and their actual values during the tests (especially near the tunnel). Small differences 17 were actually observed between the predictions of the visco-elastic and visco-elasto-plastic 18 analyses due to additional energy dissipation induced by the hysteretic response of the soil in 19 the latter cases.

(a)
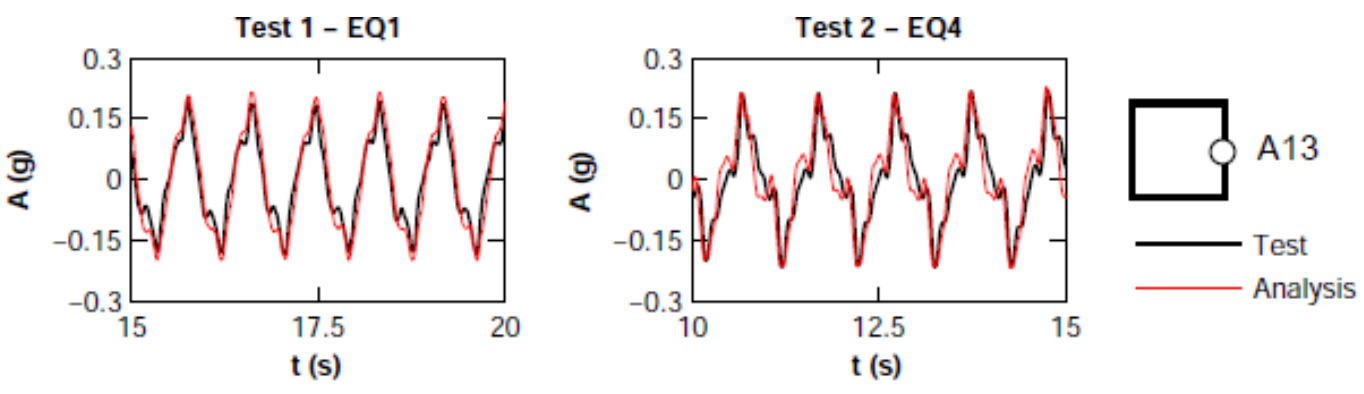

(b) Reference array - A (g)
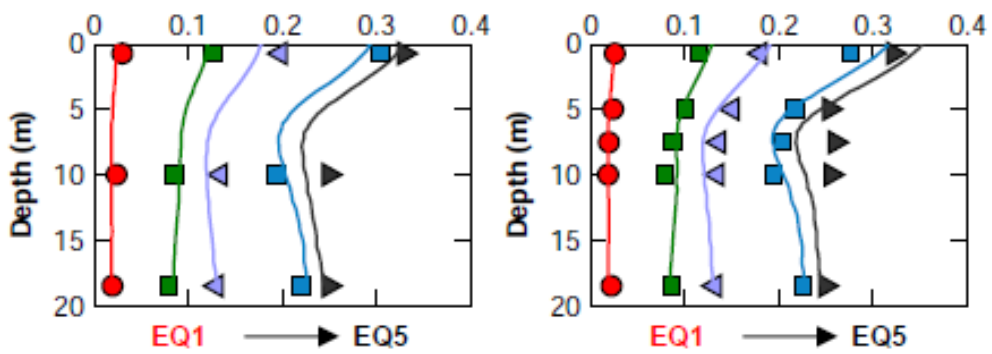

Free field array - A (g)

Tunnel array - A (g)

21 Fig. 8 (a) Time windows of acceleration time histories recorded and computed by the visco-elasto-

22 plastic analyses at the middle section of the tunnel right side-wall, (b) horizontal acceleration

23 amplification recorded and computed along the accelerometers vertical arrays for different earthquakes

24 of Test 2 (flight 1 ) 


\subsubsection{Deformed shapes of tunnels}

2 Figure 9a presents time-windows of vertical acceleration time histories recorded at the sides of the tunnel's roof slab, referring to both test cases. The time histories for the deep tunnel (Test 2) are out of phase, indicating a rocking mode of vibration for the tunnel coupled with the racking distortion. Although this observation is less evident for the shallow tunnel (Test 1), this rocking response still exists.

Numerically derived deformed shapes of the deep tunnels (numerical configuration of Test 2) are presented in Fig. 9b, referring to the time step of computed maximum racking distortion 10 above. Naturally, the rigid tunnel exhibits lower racking distortion than the flexible one. Owing 11 to the high flexibility of the flexible tunnel and the associated high soil yielding around the 12 tunnel, inward deformations of the slabs and the side-walls are predicted by the visco-elasto13 plastic analyses, in addition to the racking-rocking response mentioned above. These 14 observations a with recent findings of Cilingir and Madabhushi [17], who derived deformed 15 shapes of flexible square model tunnels embedded in dry sand during centrifuge testing, using 16 particle image velocimetry methods. Regardless of the lining flexibility, the numerical analyses revealed an increase of the rocking response of the tunnel section with the increase of the shaking amplitude. Similar observations were made for the shallow tunnels [49].

19

(a)
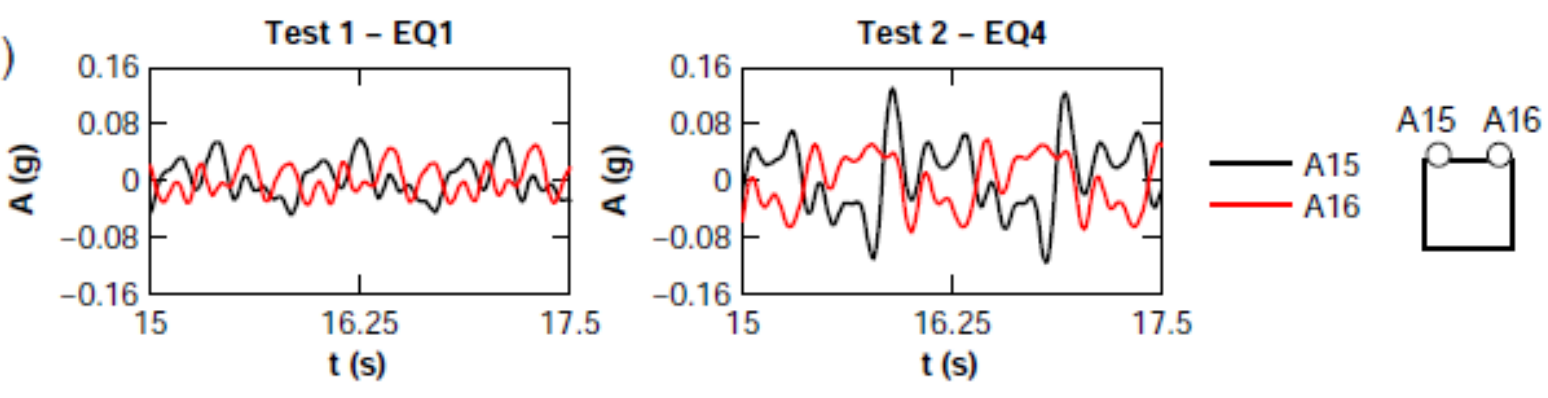

(b)
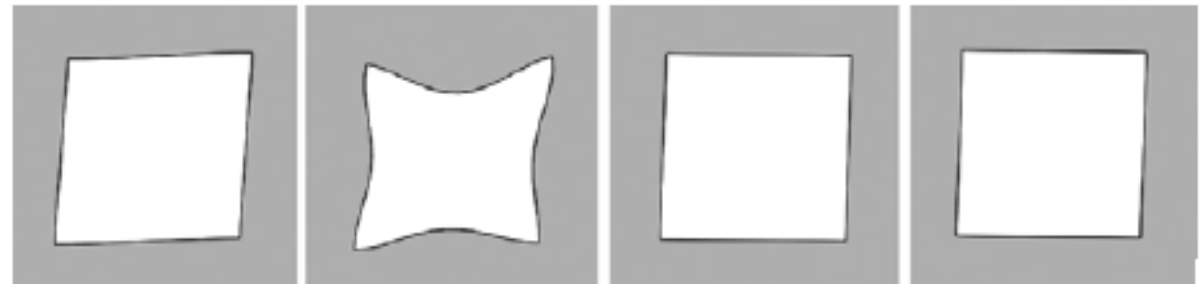

21 Fig. 9 (a) Time windows of vertical acceleration time histories recorded at the sides of the tunnel' s roof slab during the tests, (b) 'dynamic' deformed shapes of deep tunnels for the time step of the computed maximum racking distortion (Test 2 - EQ4, deformation scale $\times 60$ ) 


\section{$1 \quad$ 5.2.3 Dynamic earth pressures}

2 Figure 10 compares dynamic earth pressures time histories computed and recorded at the left

3 side-wall of the tunnel. The comparisons refer to representative results from both test cases.

4 The numerical results are computed by the visco-elasto-plastic analyses for different

5 assumptions regarding the soil-tunnel interface characteristics. Both recorded and computed

6 data reveal a post-earthquake residual response for the dynamic earth pressures, as well as

7 higher values near the rigid corners of the tunnel. The residual response that has also been

8 reported during similar centrifuge tests [16-17] is related to soil yielding and densification that

9 are taking place around the tunnel during shaking. The observed differences between the

10 recorded and the computed earth pressures may be attributed to several issues, including the

11 assumptions and calibration of the constitutive model (see section 5.1), as well as recording

12 issues related to the miniature earth pressures cells recording capabilities in the case of granular

13 dry sand. With reference to the recording issues, the relative stiffness of the sensing plate may

14 affect the readings, while there are also problems related to the grain size effect [15]. Part of

15 the differences may be due to inward deformations of the side-wall that may change slightly

16 the recording direction (small inclination of the pressure cell) compared to the 'normal'

17 direction in the numerical analysis.

(a)
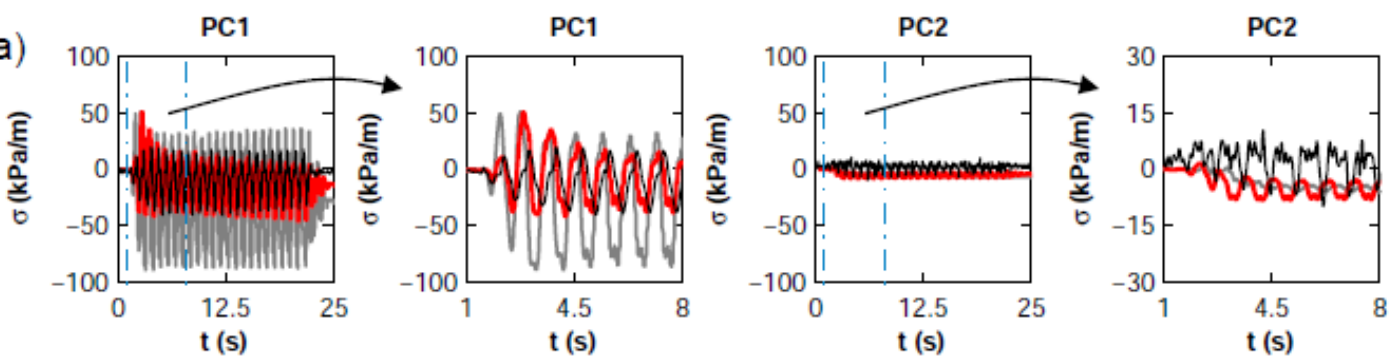

$(\bar{b})$
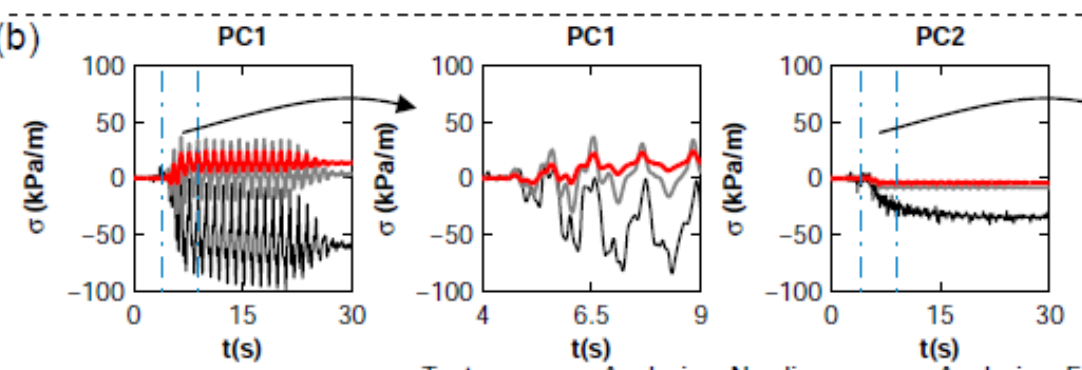

Test

$\mathrm{t}(\mathrm{s})$

Analysis - Full slip

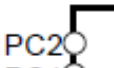
$\mathrm{PC} 1 \mathrm{C}$

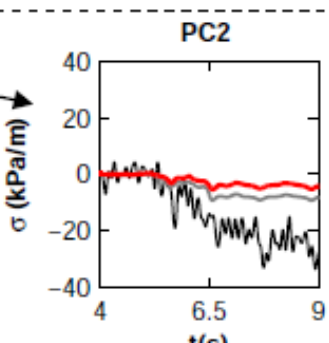

Fig. 10 Dynamic earth pressures time histories recorded and computed by visco-elasto-plastic analyses at the left side-wall; effect of the soil-tunnel interface characteristics (a) Test 1, EQ4, (b) Test 2, EQ6

The computed earth pressures around the tunnel are clearly affected by the soil-tunnel interface characteristics, with the effect being generally more evident in the reversible 
the numerical predictions of the full-slip condition analyses are closer to the recorded data. The model tunnel, which had a rather smooth external surface in the particular test, is embedded in a shallow depth and in a rather loose sand deposit. To this end, the confining pressure on the tunnel is relatively low and thus the interface is rather weak (conditions closer to full-slip). The better performance of the no-slip full bonding conditions in Test 2 should be attributed to the numerical simulation per se. Owing to the high flexibility of the lining, the lining elements exhibits large inward deformations (deformations towards the cavity) during the introduction of the gravity loads (i.e. static step of the analysis). The soil elements, surrounding the tunnel, are not capable to fully follow this deformation, due to a kind of artificial 'arching effect' related to the simulation of the soil as a continuum. In this context, a small 'artificial gap' is created between the soil and the tunnel elements for the cases where an interface is considered.

12 This numerical behavior opposes to the actual behavior in the centrifuge, where no separation 13 between the sand and the lining could occur. The gap was more evident for the deep tunnel 14 (Test 2), as the confining pressures and the associated lining inward deformations were higher 15 in this case. The analyses under full bonding conditions ensured that the soil elements were 16 following the deformation of the lining elements, thus providing numerical predictions closer to the recorded data. It is noteworthy that the above numerical weakness is expected to be reduced with the increasing lining rigidity.

19 Representative distributions of the dynamic earth pressures computed along the perimeter of 20 the tunnel by the elasto-plastic analyses are portrayed in Fig. 11, referring to both test cases. In 21 particular, the distributions refer to envelope (maximum and minimum) and simultaneous 22 values, with the latter being computed at the time step of maximum racking distortion of the tunnel (Fig. 11a), post-earthquake residual values (Fig. 11b), and dynamic increments of the earth pressures (Fig. 11c). The dynamic increments (i.e. reversible component of the earth pressure time histories) are computed as the semi-amplitude of the maximum value of the cycles in the earth pressure time histories. The numerical predictions, which are compared with the recorded data (i.e. recorded values on the left side-wall), refer to full-slip interface conditions in case of Test 1 and to no-slip tie conditions in case of Test 2. As stated above, 29 these cases provided better comparisons with the recorded response. Despite the differences 30 between the recorded data and the computed response, the overall comparison is satisfactory. 31 In particular, the earth pressures computed at the time step of maximum racking distortion of 32 the tunnel are distributed in an antisymmetric fashion along the slabs and the side-walls (Fig. 33 11a). The yielding of the surrounding soil and the associated post-earthquake residual values 34 on the earth pressures, affect the distributions altering this anti-symmetric response. This 
1 behavior is more evident during the first shakes of each flight of the centrifuge, where a large

2 part of the soil plastic strain is accumulated. The dynamic increments of the earth pressures are 3 amplified near the stiff corners of the tunnel, while they become almost negligible at the 4 middle sections of the slabs and the side-walls (Fig. 11c).
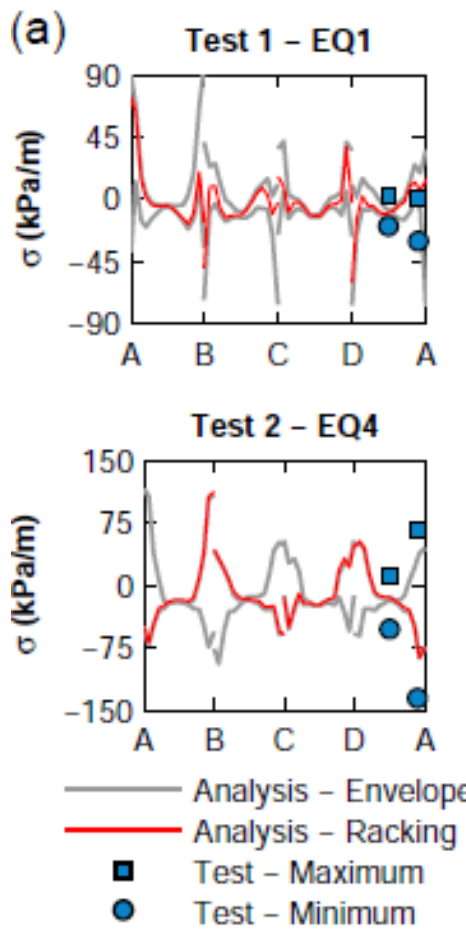

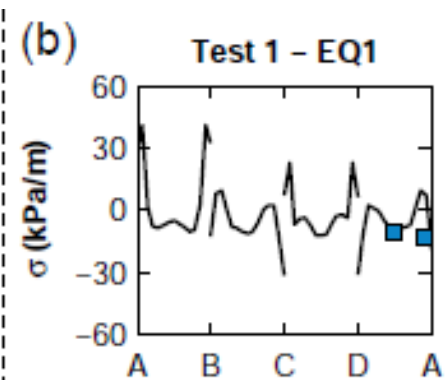

Test 2 - EQ4

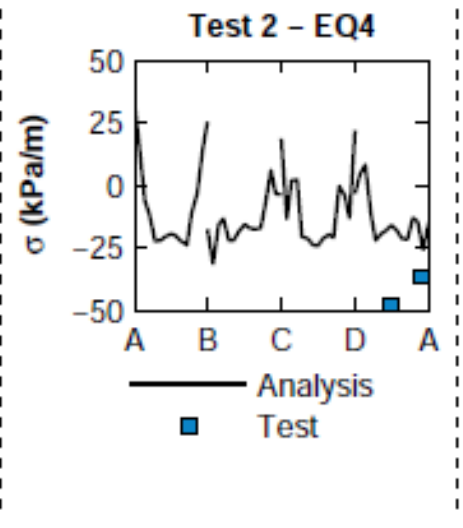

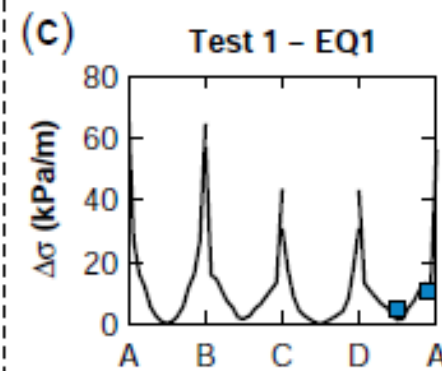

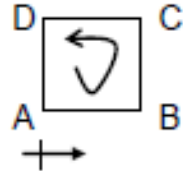

Test 2 - EQ4
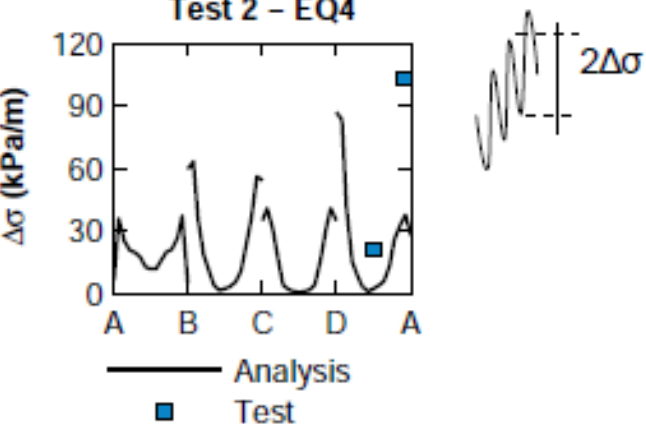

Fig. 11 Dynamic earth pressures distributions computed along the perimeter of the flexible tunnel by the visco-elasto-plastic model plotted against the recorded data, (a) envelope distributions, (b) residual distributions at the end of each shaking, (c) dynamic increment distributions

Figure 12 highlights the effects of soil-tunnel interface characteristics, soil yielding and

13 lining rigidity on the dynamic earth pressure distributions computed along the perimeter of the

14 shallow tunnels (soil-tunnel configuration of Test 1). The results, referring to both the actual

15 model tunnel and the tunnel with the increased lining thickness, are computed at the time step 16 of maximum racking distortion for different assumptions regarding the soil response and the soil-tunnel interface conditions. For elastic soil response and no-slip tie conditions at the interface, the anti-symmetric distribution of the dynamic earth pressures along the slabs and the side-walls is again verified. Full-slip interface conditions result in higher earth pressures at the corners of the tunnel compared to the tie conditions. It is worth noticing the higher dynamic earth pressures computed on the corners of the flexible tunnel compared to the rigid one. This observation is explained as follows: for a given soil-tunnel configuration, the increase of the 
1 lining flexibility results to an increase of its racking distortion, which in addition to the rocking

2 response, leads to higher earth pressures on the corners of the slabs and the side-walls (i.e.

3 higher resistance of the soil to the increased deformations). In other words, the local earth

4 pressures near the corners reach the 'passive state'.
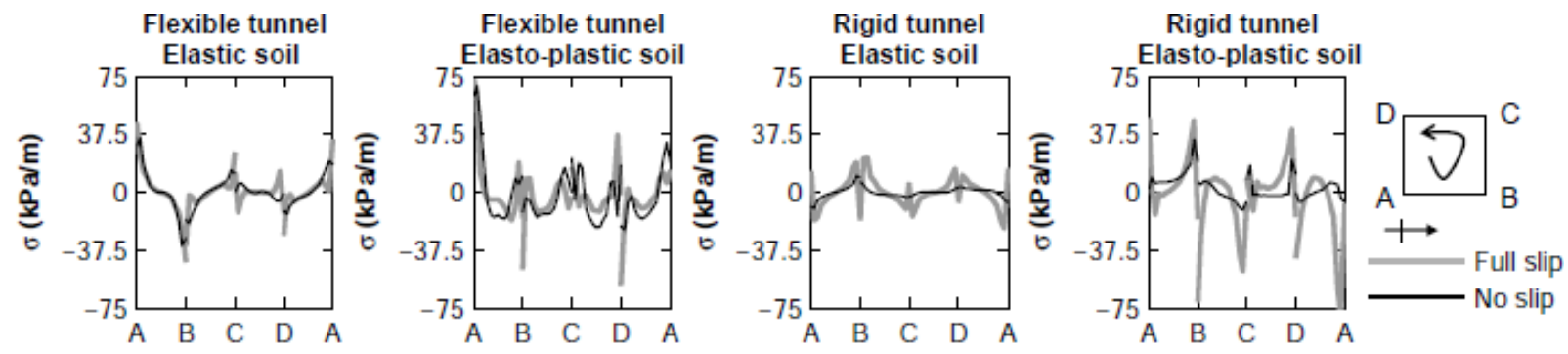

Fig. 12 Effects of soil-tunnel interface characteristics, soil yielding and lining rigidity on the dynamic earth pressures distributions computed along the perimeter of the shallow tunnels (Test 1, EQ1)

The yielding of the soil and the associated stress redistributions within the sand around the tunnel result in more complex forms for the computed earth pressures distributions. Generally, the soil yielding and therefore its effect on the dynamic earth pressures distributions are more pronounced for the flexible tunnel. Different soil-tunnel interface characteristics may result in a different soil yielding pattern around the tunnel, thus modifying the computed earth pressure distributions (Fig. 12). The above observations are verified in Fig. 13, where plots of the soil plastic strain (dynamic part) computed around shallow tunnels at the end of shaking EQ4 (Table 3) are presented. The results, which refer to both flexible and rigid tunnels, are plotted for different soil-tunnel interface characteristics. The mobilization of soil yielding is higher around the flexible tunnels, while for full-slip interface conditions this yielding is concentrated near the corners of the tunnel. The relation between the soil yielding and the soil-tunnel interface characteristics has also been reported by Huo et al. [25]. Similar observations were made for the deep tunnels, with the computed soil plastic strain been however lower.

It is common in engineering practice to perform the seismic analysis of a tunnel using the so-called simplified equivalent static analysis method [29]. The soil-tunnel system is analysed using a frame-spring model, while the earth pressures are modelled using prescriptions, referring to retaining walls, i.e. the Mononobe Okabe approach [38-39] or regulations referring to non-deformable rigid walls [11]. To verify the validity of this approach, the dynamic part of the earth pressures predicted by the above simplified methods is compared with the dynamic part of the earth pressures computed by the rigorous numerical analyses. The direct comparison of the predictions of pseudo-static limit equilibrium methods (i.e. M-O approach) with the 
1 results of the full dynamic analysis, which does not refer to limit states but instead try to

2 replicate the 'actual' dynamic response of the soil-tunnel system, is not conceptually right.

3 However, the following comparisons are useful to highlight the limitations of common design

4 approaches.

(a)

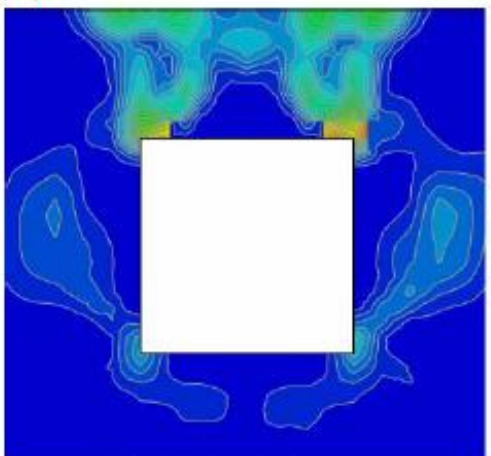

PE, plastic strain

$0.050 \quad 0.044$ (b)

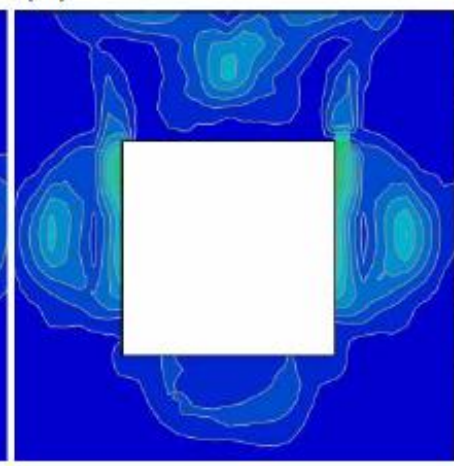

0.038 (c)

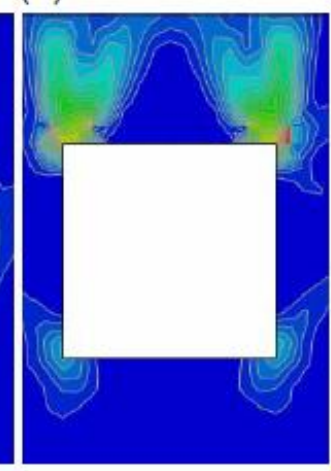

$0.025 \quad 0.019$

0.013 (d)

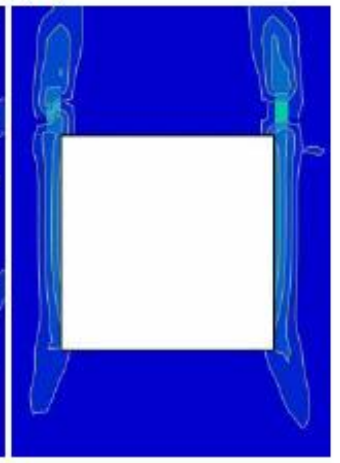

$0.006 \quad 0.000$
6

Fig. 13 Soil plastic strain (dynamic part) computed around the shallow tunnels at the end of shaking EQ4 (Test 1), (a) flexible tunnel, full-slip interface conditions, (b) flexible tunnel, no-slip interface conditions, (c) rigid tunnel, full-slip interface conditions, (d) rigid tunnel, no-slip interface conditions

Figure 14 portrays representative comparisons of the dynamic part of the earth pressures, computed by the various design approaches along the side-walls of the deep tunnels (i.e. numerical model configuration of Test 2) for shaking motion EQ4 (Table 3). For the sake brevity the comparisons are presented only for the left side-walls; similar observations were made for the right ones. The numerical results are computed for the specific shaking motion (separate analysis), assuming no-slip tie conditions and elastic or elasto-plastic soil response. They refer to both the flexible and the rigid tunnel and correspond to absolute maximum envelope values. An average ground acceleration is used for the computation of the earth pressures with the simplified methods, which is estimated by the elasto-plastic analysis at the soil 'free-field', corresponding to the same depth with the tunnel. To implement the M-O approach, the friction angle $\varphi$ is assumed equal to $33^{\circ}$ (critical friction angle of the sand fraction), while the soil-wall interface friction angle $(\delta)$ is assumed equal to the sand friction angle. The earth pressures, computed following the M-O approach, refer to both active and passive conditions and are distributed on the walls assuming different patterns, as displayed in the figure (e.g. uniform distribution, triangular distribution). 


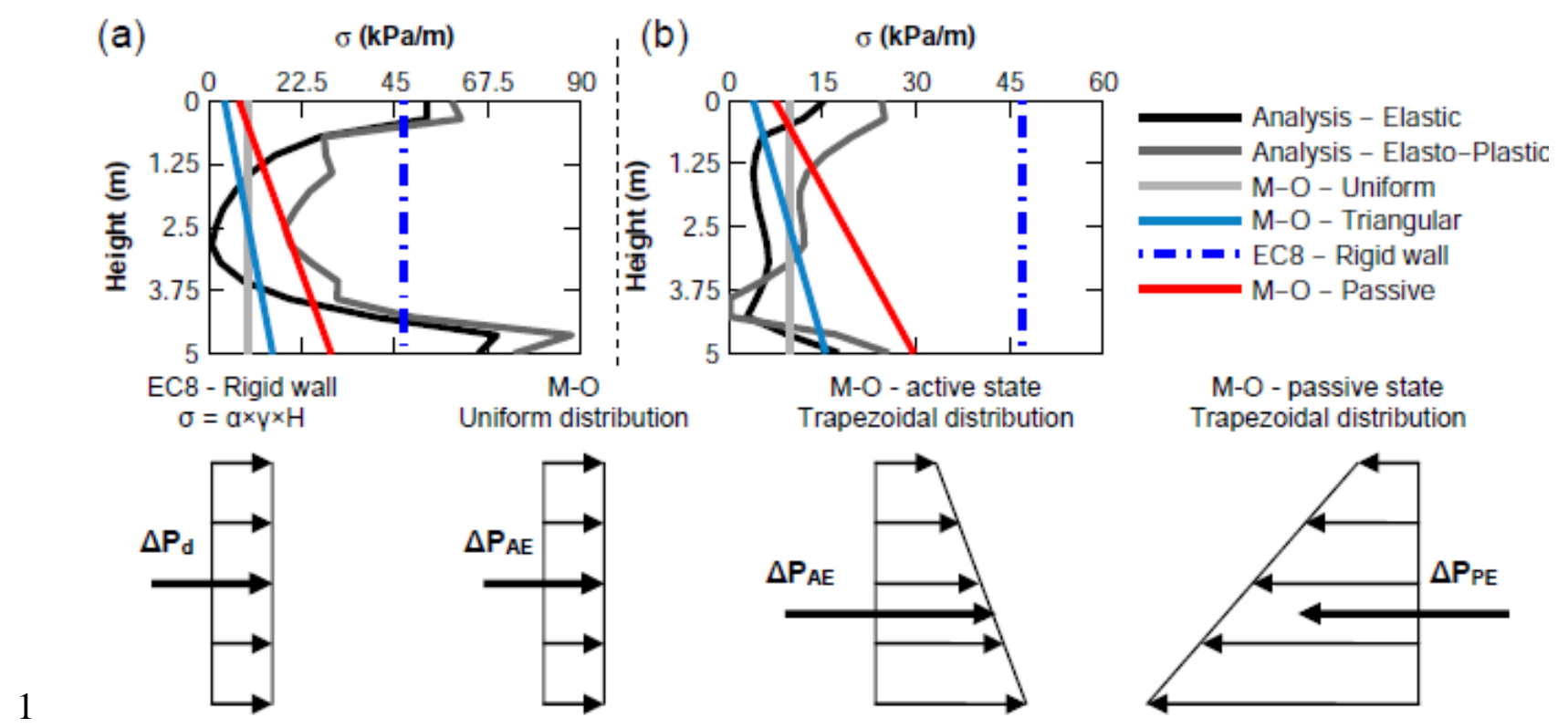

(b) $\quad \sigma(\mathrm{kPa} / \mathrm{m})$

2 Fig. 14 Dynamic part of the earth pressures computed by the numerical analyses and the simplified 3 approaches on the left side-walls of the deep tunnels for EQ4 (a) flexible tunnel, (b) rigid tunnel

Generally, the elasto-plastic analyses provide higher earth pressures at the middle sections of the side-walls compared to the elastic ones. This is attributed to the soil yielding and the associated high residual values on the earth pressures predicted by the elasto-plastic analyses at

8 these locations. The earth pressures (dynamic part) computed following the prescriptions of the

9 EC8 for non deformable rigid walls [11] are more conservative than the M-O approach.

10 However, the results of both simplified approaches deviate considerably from the numerical 11 predictions. Evidently, the simplified approaches are not capable to reproduce the actual earth 12 pressures distribution pattern (e.g. concentrations of the earth pressures at the corners). The 13 complex racking-rocking deformation mode, mobilized by square tunnels during shaking, and 14 the associated dynamic earth pressures due to this deformation pattern, can not be simulated by 15 the simplified limit equilibrium methods that are inspired from the kinematic response of 16 retaining walls. In this context, the simplified approaches for the evaluation of the dynamic 17 earth pressures should probably be avoided or used with caution to roughly evaluate the 18 magnitude of the dynamic part of the earth pressures during shaking. Similar observations were 19 made for the shallow tunnels (numerical configuration of Test 1).

\section{$21 \quad$ 5.2.4 Soil dynamic shear stresses}

22 Naturally, the soil dynamic shear stresses around the tunnel are increased with the increase of 23 tunnel burial depth and sand densification, while the friction developed during shaking along 24 the soil-tunnel interface, plays an important role on the shear stress distribution and magnitude. 
1 Fig. 15 portrays typical soil dynamic shear stress distributions computed around the perimeter

2 of the deep tunnels (numerical configuration of Test 2), referring to time step of maximum

3 racking distortion. The results, which are plotted for different assumptions regarding the soil-

4 tunnel interface characteristics, the soil non-linear response and the lining rigidity, are

5 compared with the soil dynamic shear stresses computed at soil 'free-field' conditions at the

6 same depth with the tunnel. For full-slip conditions, the computed soil shear stresses are

7 increased at the tunnel corners, while they are significantly reduced along the middle sections

8 of the slabs and side-walls. An increase of the friction along the soil-tunnel interface leads to

9 an increase of the soil shear stresses along the middle sections. Similar to the dynamic earth

10 pressures, soil yielding affects the computed soil shear stresses, making the distributions more

11 complex. Interestingly, the 'free-field' soil shear stresses computed at the same depth with the

12 tunnel are compared reasonably well with the soil shear stresses computed along the perimeter

13 of the tunnel for the no-slip conditions. The comparisons are better for an elastic soil response

14 and for the rigid tunnel. Similar conclusions were drawn for the shallow tunnels.

15
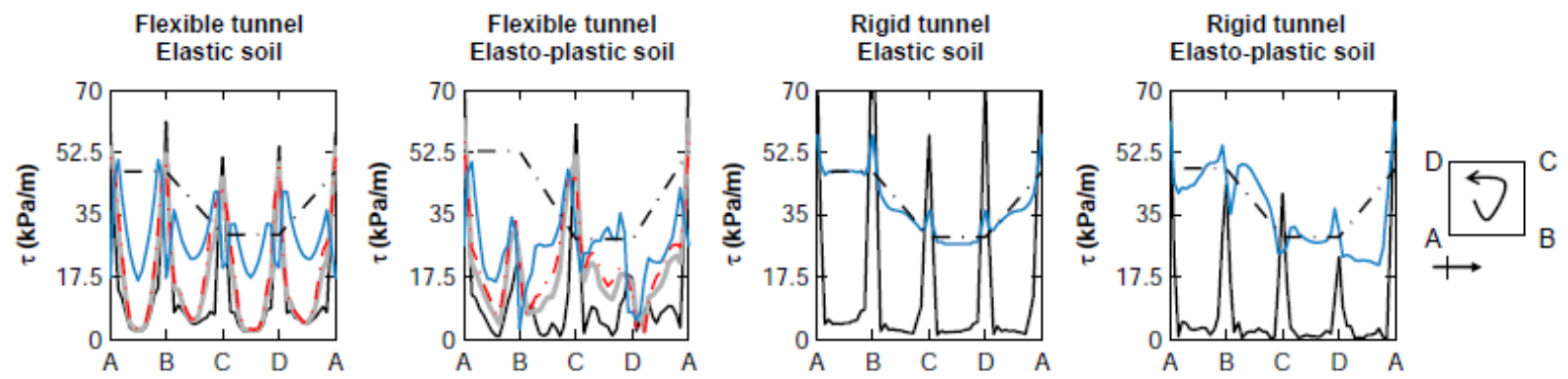

Full slip

$\mu=0.4 \cdot \boldsymbol{U} \cdot \boldsymbol{U} \cdot \boldsymbol{\mu}=0.8$

No slip $\cdot \boldsymbol{-} \cdot \boldsymbol{-} \cdot$ Soil free field

17 Fig. 15 Effects of soil-tunnel interface characteristics, soil yielding response and lining rigidity on the soil dynamic shear stress distributions computed along the perimeter of the deep tunnels (Test 2, EQ4)

\subsubsection{Dynamic lining forces}

This section summarizes the main findings regarding the dynamic lining forces of the tunnels, which are crucial for the detailing of the lining sections. The crucial effects of soil-tunnel relative flexibility, soil-tunnel interface conditions and soil yielding on the dynamic lining forces are highlighted and discussed. In line with the experimental data, the numerical results reveal post-earthquake residual values for the lining forces of the flexible tunnels. The increase of the lining rigidity results in a significant reduction of this permanent response. Soil-tunnel interface characteristics are found to affect significantly the dynamic axial forces, while the effect is less evident for the dynamic bending moment. These observations are presented in 


\section{$1 \quad$ 5.2.5.1 Dynamic bending moment}

2 Figure16a compares dynamic bending moment time histories recorded and computed by the 3 visco-elasto-plastic analyses for no-slip tie conditions, referring to Test 2. Both the 4 experimental data and the numerical predictions indicate a post-earthquake residual response,

5 similar to that of the dynamic earth pressures. As stated above, this response is associated with

6 the soil yielding and densification of sand around the tunnel, which are taking place during

7 shaking. The deviations between the experimental data and the numerical predictions should be 8 attributed to the simplification of the constitutive model, to calibration issues of the above 9 model, as well as to uncertainties related to the calibration of strain gauges.
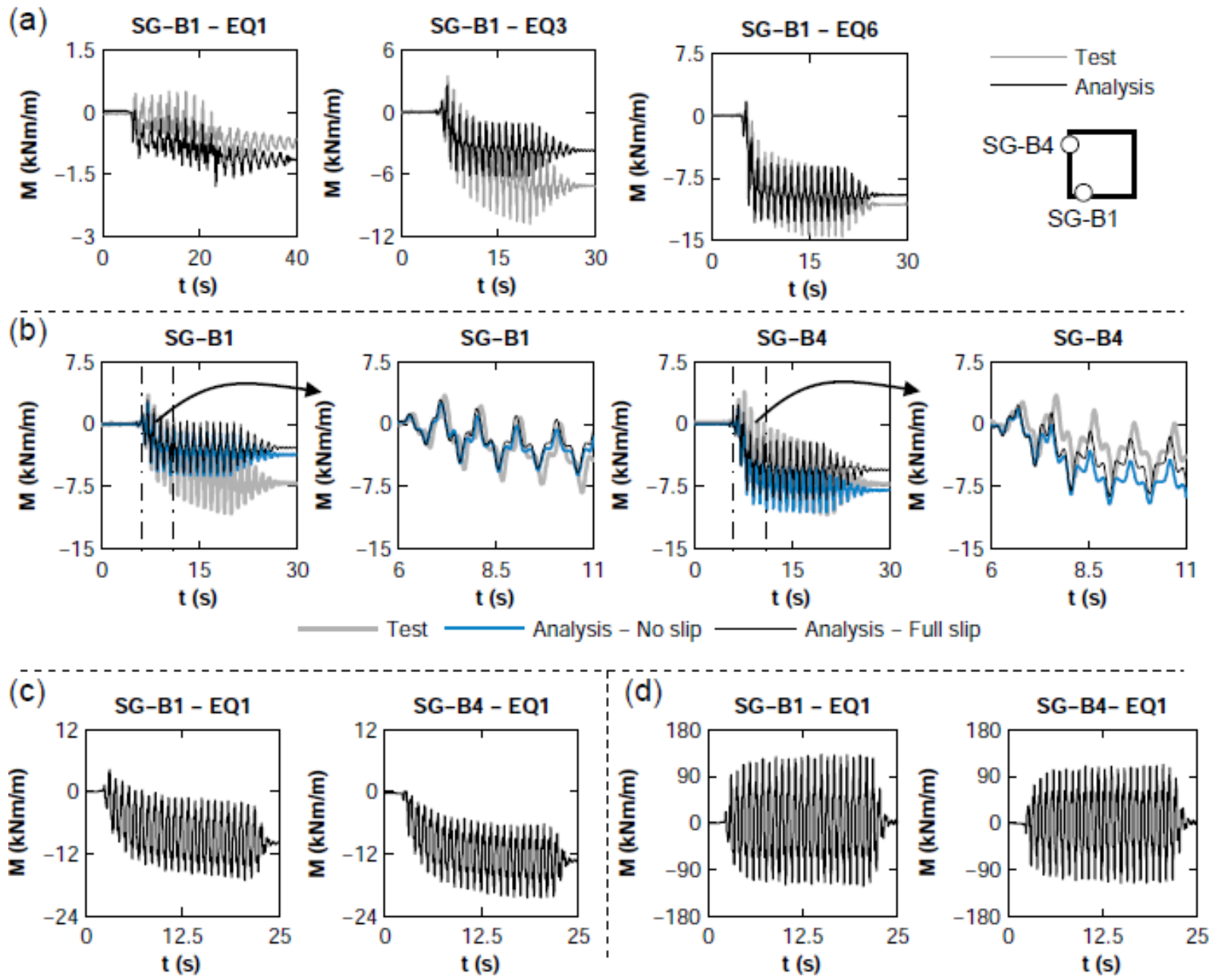

12 Fig. 16 (a) Comparisons of dynamic bending moment time histories recorded and computed at SG-B1

13 location for different shakings (Test 2), (b) effect of the soil-tunnel interface characteristics on the

14 computed dynamic bending moments (Test 2, EQ3), (c) dynamic bending moments computed at the

15 strain gauges locations referring to the shallow model tunnel (Test 1), (d) dynamic bending moments

16 computed at the strain gauges locations referring to the rigid shallow tunnel (Test 1) 
The effect of soil-tunnel interface characteristics on the computed dynamic bending moments is highlighted in Fig. 16b. The soil-tunnel interface characteristics affect the soil yielding (i.e. Fig. 13) and to some extent the sand stiffness around the tunnel. In this regard, it is expected that the soil-tunnel interface characteristics will affect the computed bending moments both in terms of residuals and dynamic increments. The effect of interface conditions is generally higher for the dynamic bending moment compared to the dynamic earth pressures.

Similar to the deep tunnel, the dynamic bending moment time histories predicted by the visco-elasto-plastic analysis for the shallow flexible model tunnel (Test 1) revealed postearthquake residual values (Fig. 16c). Much larger dynamic increments are predicted for dynamic bending moment of the rigid tunnel compared to the flexible one, while the postearthquake residual response is almost eliminated (Fig. 16d). The latter observation is in line with the reduction of soil yielding around the rigid tunnel (e.g. Fig. 13).

Figure 17 portrays dynamic bending moment distributions computed by the elasto-plastic analyses along the perimeter of the deep model tunnel (Test 2). The distributions refer to envelope and simultaneous values; the latter corresponding to the time step of maximum racking distortion of the tunnel (Fig. 17a), post-earthquake residual values (Fig. 17b) and dynamic increments of the bending moment (Fig. 17c). Similar to the dynamic earth pressures, the dynamic increments (i.e. reversible component of the dynamic bending moment) are computed as the semi-amplitudes of the maximum values of the cycles in the bending moment time histories. Generally, the numerical results, which refer to no-slip tie conditions, are in a good agreement with the recorded data. The distributions, referring to the time step of maximum racking distortion of the tunnel, are quite complex and barely follow the expected anti-symmetric distribution along the slabs and the side-walls. This response is related to the increased soil yielding mobilization around the tunnel and the associated significant postearthquake residual values mentioned above (e.g. Fig. 17b).

Figure 18a illustrates the effects of lining rigidity, soil yielding and soil-tunnel interface conditions on the dynamic bending moment distributions computed along the perimeter of the deep tunnels. The distributions refer to the time step of maximum racking distortion of the

29 tunnel. For elastic soil response, the dynamic bending moments present an anti-symmetric 30 distribution pattern along the slabs and the side-walls. The non-linear phenomena (i.e. soil 31 yielding and/or slippage along the soil-tunnel interface) modify the bending moment 32 distributions, leading to more complex patterns. This is more evident for the flexible tunnel.

33 Full bonding conditions result in higher dynamic bending moments at the corners of the tunnel 34 compared to the full-slip interface conditions. 

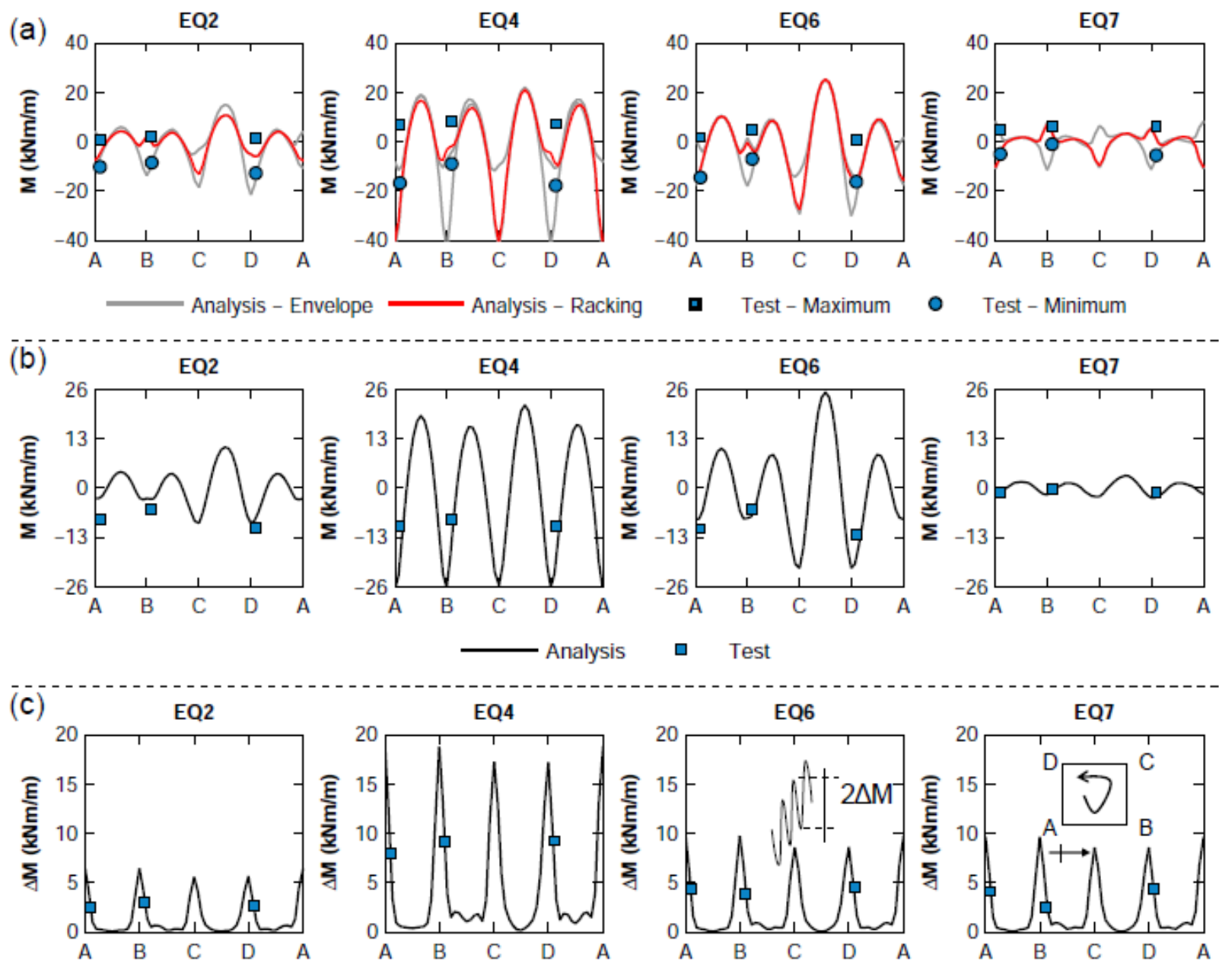

Analysis

- Test

2 Fig. 17 Dynamic bending moment distributions computed along the perimeter of the deep model tunnel (Test 2) plotted against the recorded data, (a) envelope distributions, (b) residual distributions at the end of shaking, (c) dynamic increment distributions

Similar observations are made comparing the dynamic increments of the bending moments (Fig. 18b). These increments are increased near the stiff corners of the tunnel, with the no-slip conditions resulting in higher increments at these locations. Comparing the results of visco-

9 elastic and visco-elasto-plastic analyses, it is seen that the effect of soil yielding on the bending moment increments is reduced. Similar observations were made for the shallow tunnels.

\subsubsection{Dynamic axial forces}

13 The recorded dynamic axial forces on the side-walls were out of phase (i.e. [47]), verifying the

14 rocking response of the tunnel mobilized during shaking. The numerical analyses also verified

15 the above response. 

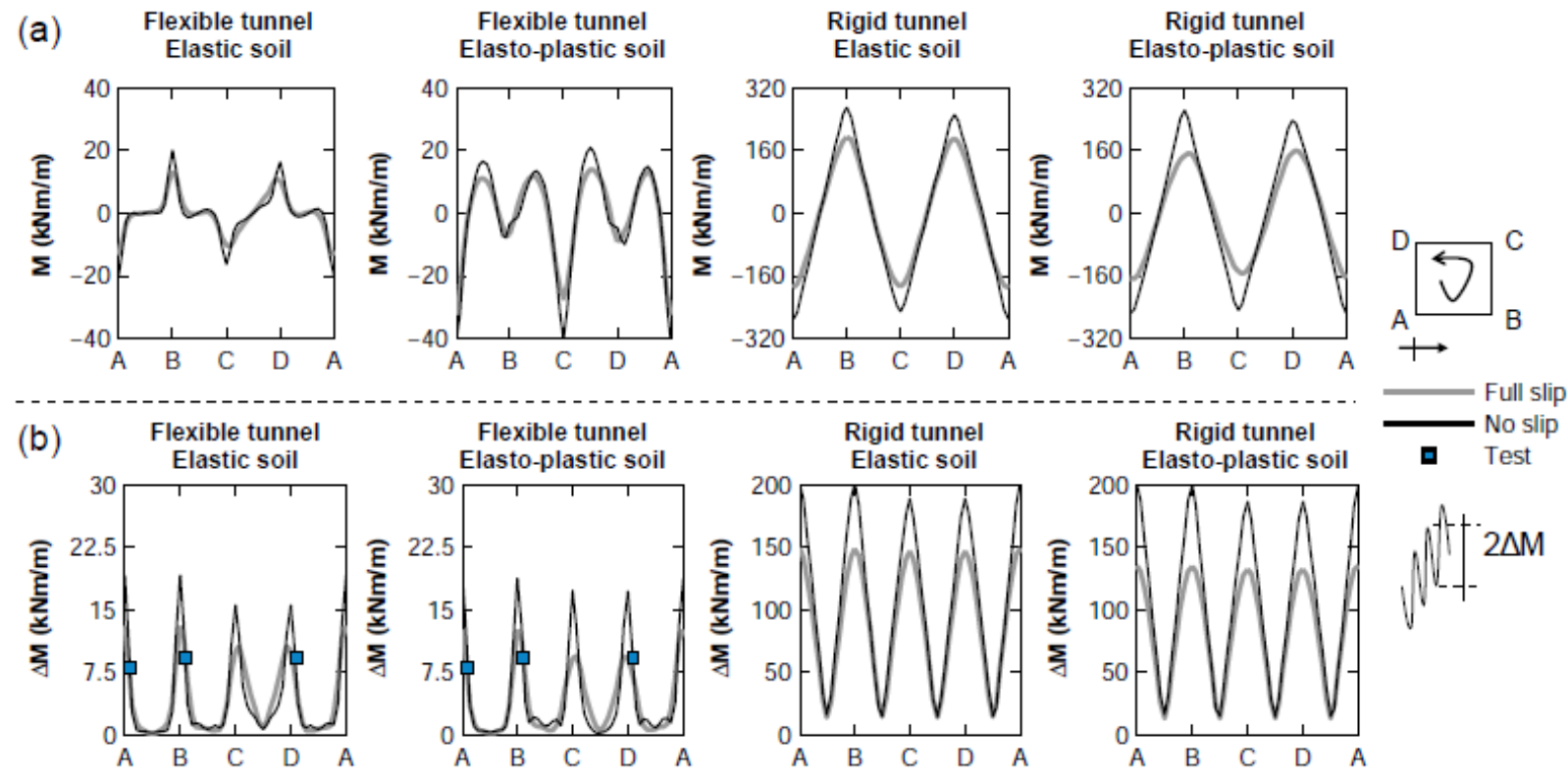

2 Fig. 18 Effects of soil-tunnel interface characteristics, soil yielding and lining rigidity on: (a) the dynamic bending moment distributions computed along the perimeter of the deep tunnels at the time step of maximum racking distortion of the tunnel, (b) the bending moment dynamic increment distributions computed along the perimeter of the deep tunnels (Test 2, EQ4)

Figure19a compares dynamic axial force time histories recorded and computed by the viscoelasto-plastic analyses for no-slip tie conditions, referring to Test 2. The numerical predictions are plotted for no-slip tie conditions. Similar to the dynamic bending moments, residual values are recorded and computed for the axial forces. These residuals that are generally reduced compared to the ones of the bending moment, are larger along the slabs. The observed deviations between the computed and the recorded response should be attributed again to the constitutive model and calibration issues mentioned above.

Contrary to the dynamic bending moment, the effect of interface conditions is more important for the dynamic axial force (Fig. 19b). Indeed, the numerical predictions for full-slip conditions indicate much lower dynamic increments for the dynamic axial forces. For the specific test case (Test 2), the numerical predictions for no-slip conditions are closer to the recorded data. This observation is in line with the observations made for the dynamic earth pressures.

20 Similar post earthquake residual values were computed for the dynamic axial forces of the

21 shallow tunnel. The increase of the lining thickness (i.e. rigid tunnel) led to an increase of the 22 dynamic increments of the axial forces, as well as in a reduction of the post-earthquake residual 23 values. 

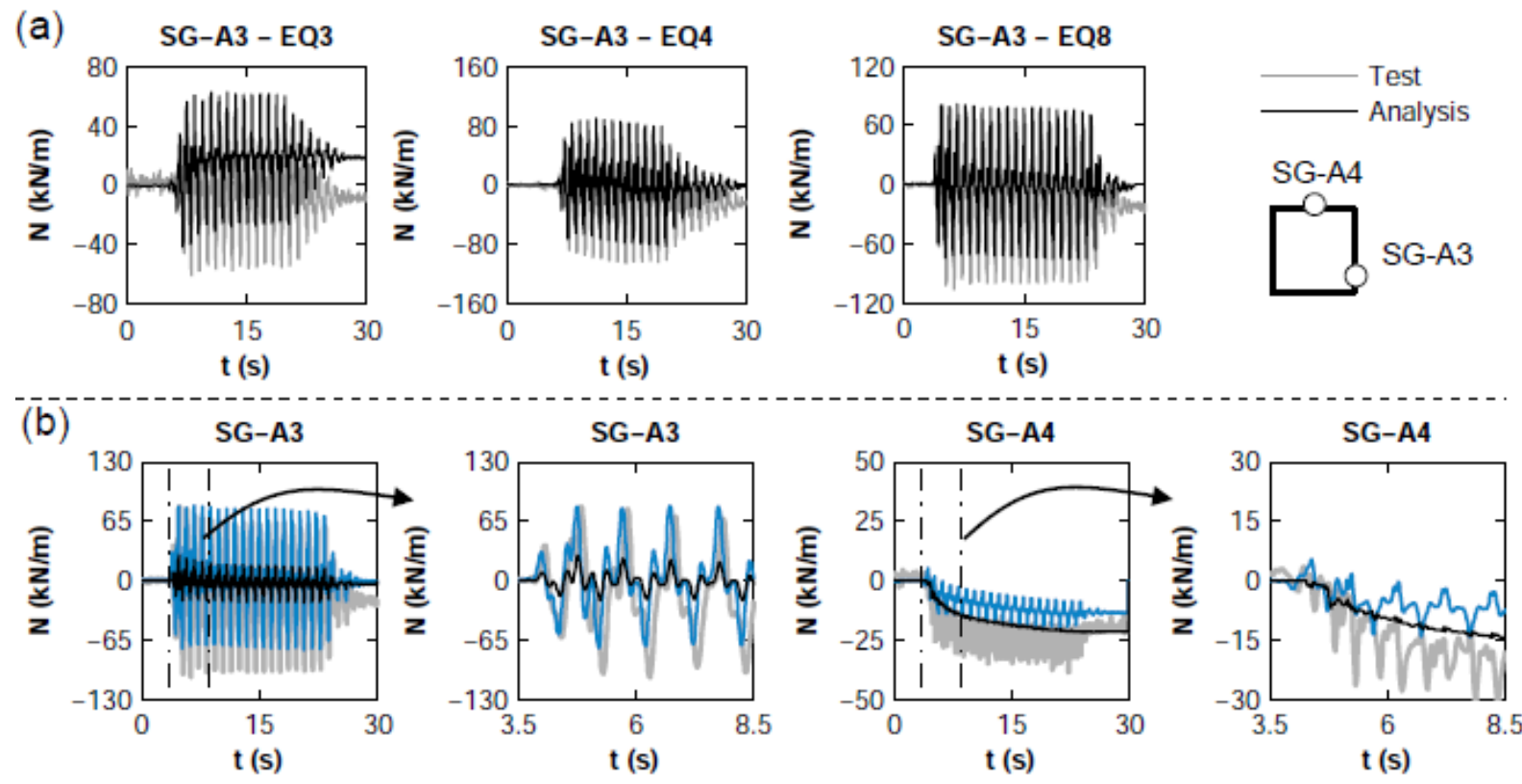

$$
\text { Test } \longrightarrow \text { Analysis - No slip — Analysis - Full slip }
$$

2 Fig. 19 (a) Comparisons of dynamic axial forces time histories recorded and computed at SG-A3

3 location for different shakings (Test 2), (b) effect of soil-tunnel interface characteristics on the 4 computed dynamic axial forces (Test 2, EQ8)

Figure 20 presents dynamic axial force distributions computed by the visco-elasto-plastic analysis along the perimeter of the deep model tunnel (Test 2). In particular, Fig. 20a illustrates envelope and simultaneous distributions, with the latter being computed at the time step of

9 maximum racking distortion of the tunnel. Fig. 20b portrays post-earthquake residual 10 distributions of the dynamic axial force, whereas in Fig. 20c distributions of the dynamic 11 increment of the axial force are depicted. The numerical predictions, referring to no-slip 12 conditions, are in a good agreement with the recorded data. The dynamic axial forces, 13 computed at the time step of maximum tunnel racking distortion, are distributed in an anti14 symmetric fashion along the slabs and the side-walls (Fig. 20a). Any deviations from this anti15 symmetric pattern should be attributed to the non-linear phenomena associated with the soil 16 yielding and the soil-tunnel interface response (i.e. sliding along the interface).

17 The effects of lining rigidity, soil yielding and soil-tunnel interface conditions on the 18 dynamic axial force distributions are highlighted in Fig. 21a. The results, referring to the time 19 step of maximum racking distortion, verify the significant effect of interface conditions on the 20 dynamic axial forces. As stated above, no-slip conditions result in antisymmetric distributions 21 of the dynamic axial forces along the slabs and the side-walls. For full-slip interface 22 conditions, on the other hand, the dynamic axial forces are significantly reduced, while they are 
1 distributed uniformly along the slabs and the side-walls. Soil yielding may alter the axial forces

2 distributions and magnitudes, especially in case of flexible tunnels.

3 Similar conclusions are drawn, by comparing the axial force dynamic increment

4 distributions computed for different soil-tunnel interface characteristics (Fig. 21b). For no-slip

5 conditions, the computed dynamic increments are increased significantly near the corners of

6 the tunnel. For full-slip conditions, the distributions become almost uniform along the slabs

7 and side-walls, with the values being significantly lower compared to the fully bonded

8 conditions.

9
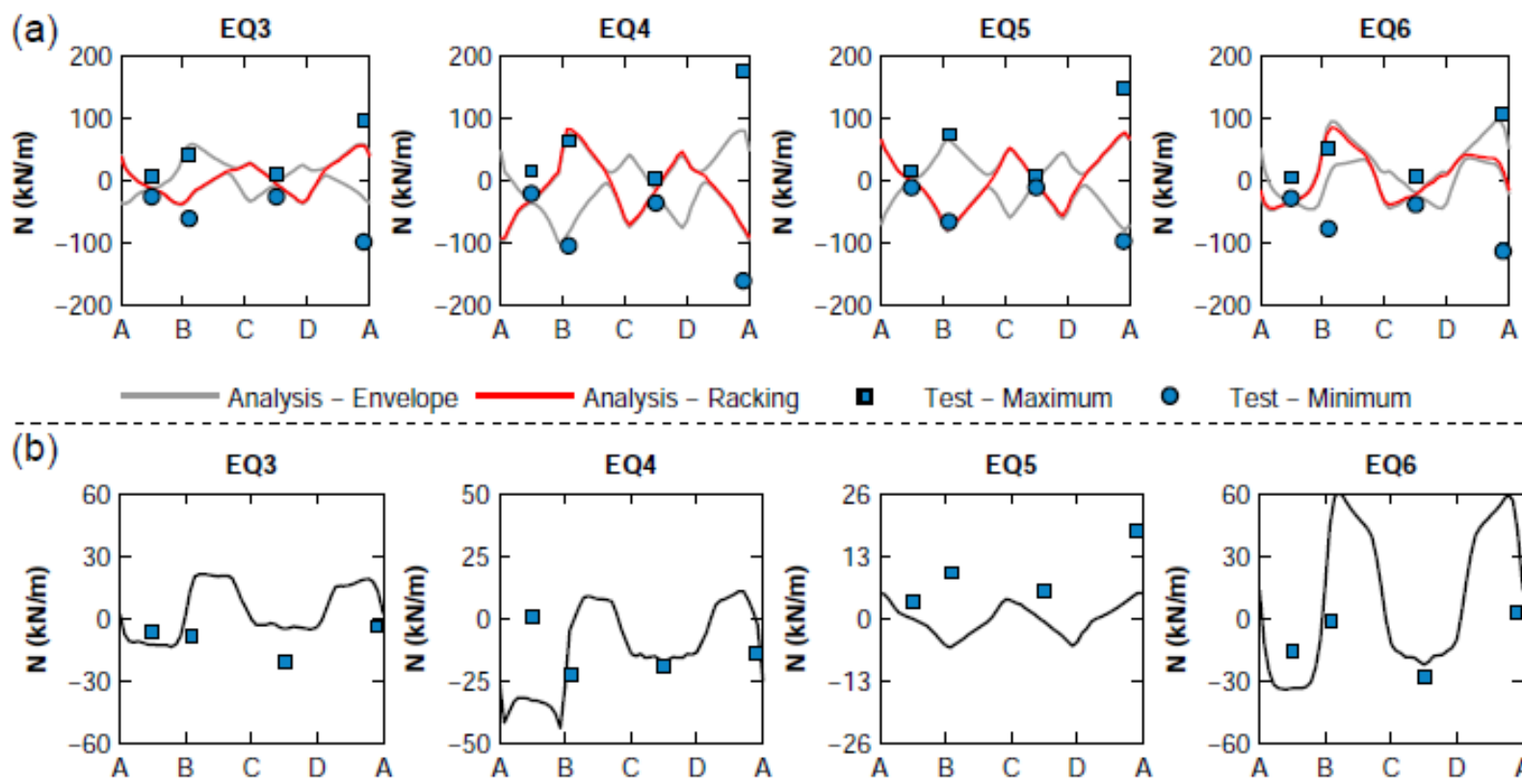

- Test - Maximum

- Test - Minimum
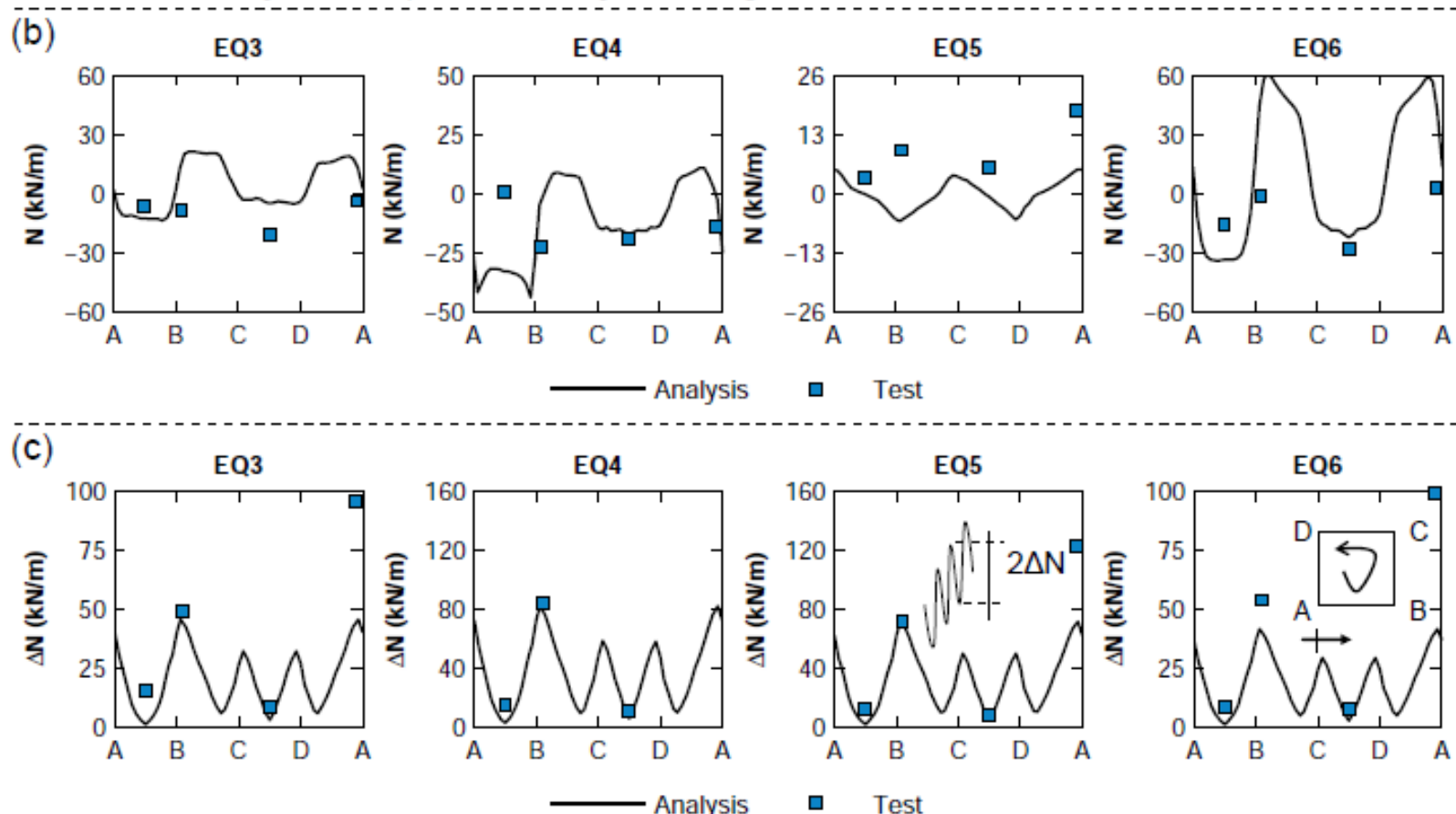

Fig. 20 Dynamic axial force distributions computed along the perimeter of the deep model tunnel (Test 2) plotted against the recorded data, (a) envelope distributions, (b) residual distributions at the end of shaking, (c) dynamic increment distributions 

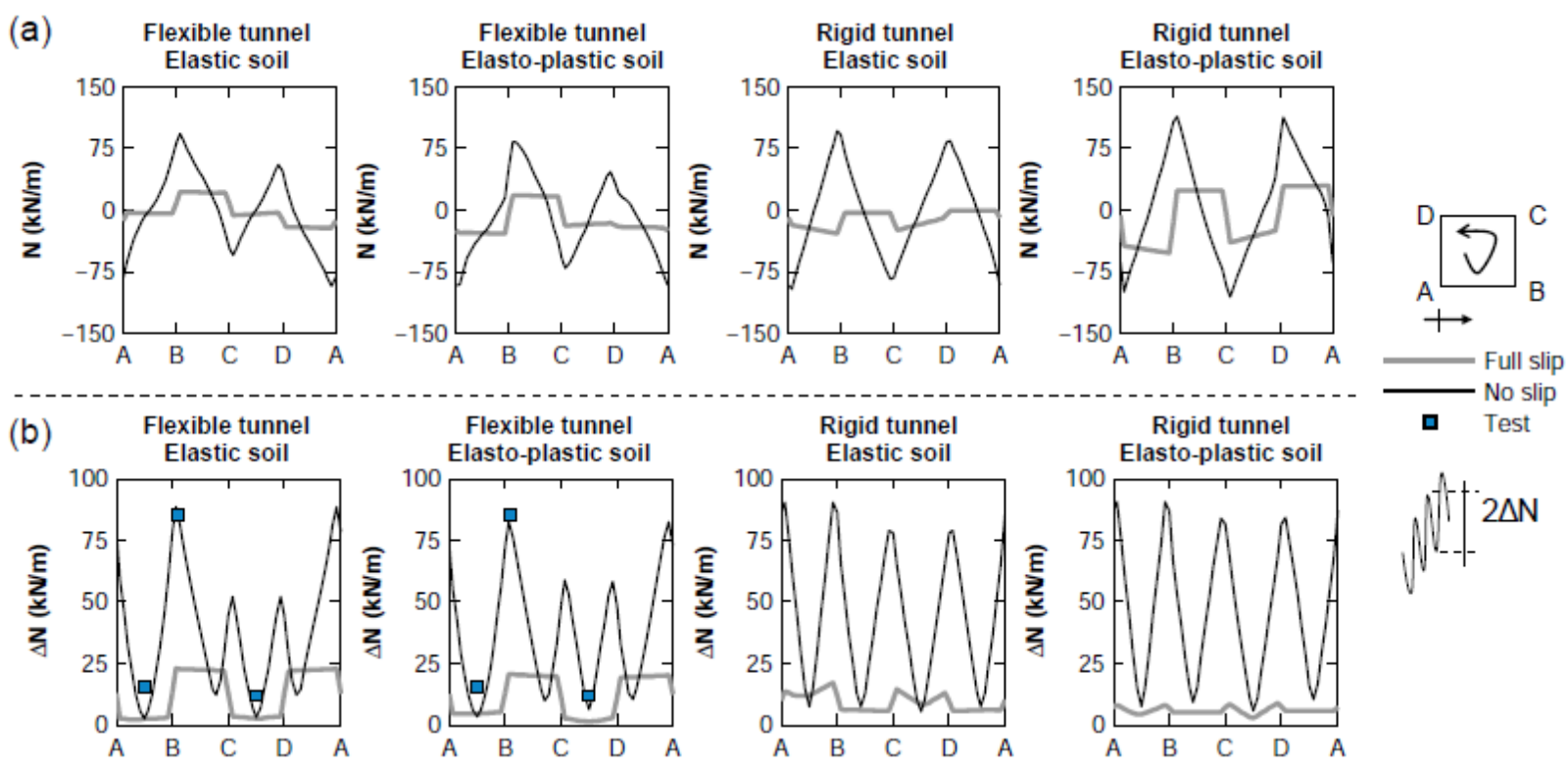

Fig. 21 Effects of soil-tunnel interface characteristics, soil yielding and lining rigidity on: (a) the dynamic axial force distributions computed along the perimeter of the deep tunnels at the time step of maximum racking distortion of the deep tunnel, (b) the axial force dynamic increment distributions computed along the perimeter of the deep tunnels (Test 2, EQ4)

\section{Conclusions}

Important aspects of the seismic response of square tunnels in sand were investigated by means of dynamic centrifuge testing and numerical analysis. The key findings of this study are summarized in the following:

- The comparisons between the predictions of the 2D and the $3 \mathrm{D}$ numerical analyses verified the hypothesis of plane strain conditions adopted in the $2 \mathrm{D}$ numerical modelling.

- The numerical models reproduced the recorded inertial response of the soil-tunnel system with reasonable engineering accuracy. Minor differences between the recorded data and the numerical predictions are attributed to calibration issues, related to the

- Both the numerical and the experimental data revealed a rocking mode of vibration for the flexible model tunnel coupled with the racking distortion. In addition, inward deformations (i.e. deformations towards the cavity) of the slabs and the side-walls were predicted by the elasto-plastic analyses. The increase of the lining rigidity resulted in a decrease of the racking distortion and the inward deformation of the slabs and the sidewalls, while the rocking response still existed. 
- The soil yielding and densification that took place around the tunnel during shaking resulted in post-earthquake residual values on the recorded dynamic earth pressures and the dynamic lining forces. The visco-elasto-plastic numerical analyses revealed similar tendencies. Moreover, the numerical analyses revealed post-shaking values on the soil shear stresses computed around the perimeter of the tunnel. These response characteristics that were increased with the flexibility of the lining (i.e. higher for the

on the response), which are partially or totally disregarded by the simplified quasi-static analysis methods that are commonly used in the seismic design of tunnels, can be treated accurately only with a full dynamic numerical analysis of the soil-tunnel system. Besides the rigorous analysis, the method may allow for a detailed sensitivity analysis, investigating, among other parameters, the crucial effect of different soil-tunnel interface conditions on the seismic response of the soil-tunnel system.

\section{Acknowledgements}

24 The research leading to these results has received funding from the European Community's 25 Seventh Framework Programme [FP7/2007-2013] for access to the Turner Beam Centrifuge, 26 Cambridge, UK under grant agreement $\mathrm{n}^{\circ} 227887$ [SERIES: Seismic Engineering Research 27 Infrastructures for European Synergies; www.series.upatras.gr/TUNNELSEIS]. The Authors acknowledge Dr. Charles Heron for his valuable help during the experimental program. The technical support received by the Technicians of the Schofield Centre is gratefully acknowledged. 


\section{References}

1. ABAQUS. Theory and analysis user's manual, version 6.12. Providence: Dassault Systèmes SIMULIA; 2012.

2. Abuhajar O, El Naggar H, Newson T. Seismic soil-culvert interaction. Can Geotech J 2015; 52:1-19.

3. Amorosi A, Boldini D. Numerical modelling of the transverse dynamic behavior of circular tunnels in clayey soils. Soil Dynam Earthquake Engng 2009; 59(6):1059-1072.

4. Anastasopoulos I, Gerolymos N, Drosos V, Kourkoulis R, Georgarakos T, Gazetas G. Nonlinear response of deep immersed tunnel to strong seismic shaking. J Geotech Geoenviron Engng 2007; 133(9):1067-1090.

5. Anastasopoulos I, Gerolymos N, Drosos V, Georgarakos T, Kourkoulis R, Gazetas G. Behavior of deep immersed tunnel under combined normal fault rupture deformation and subsequent seismic shaking. Bull Earthquake Engng 2008; 6(2):213-239.

6. Bardet JB, Ichii K, Lin CH. EERA: a computer program for equivalent-linear earthquake site response analyses of layered soil deposits. Los Angeles: University of Southern California, Department of Civil Engineering; 2000.

7. Bilotta E, Lanzano G, Madabhushi SPG, Silvestri F. A numerical Round Robin on tunnels under seismic actions. Acta Geotechnica 2014; 9(4):563-579.

8. Bobet A, Fernandez G, Huo H, Ramirez J. A practical iterative procedure to estimate seismic-induced deformations of shallow rectangular structures. Can Geotech J 2008; 45:923-938.

9. Bobet A. Drained and undrained response of deep tunnels subjected to far-field shear loading. Tunnell Underground Space Technol 2010; 25(1):21-31.

10. Brennan AJ, Thusyanthan NI, Madabhushi SPG. Evaluation of shear modulus and damping in dynamic centrifuge tests. J Geotech Geoenviron Engng 2005; 131(12):1488-1497.

11. CEN. Eurocode 8: Design of structures for earthquake resistance - Part 5: Foundations, retaining structures and geotechnical aspects. EN 1998-5:2004. Brussels: European Committee for Standardization; 2004.

12. Chen G, Wang Z, Zuo X, Du X, Gao H. Shaking table test on seismic failure characteristics of a subway station structure in liquefiable ground. Earthquake Engng Structl Dynam 2013; 42:1489-1507.

13. Chian SC, Madabhushi SPG. Effect of buried depth and diameter on uplift of underground structures in liquefied soils. Soil Dynam Earthquake Engng 2012; 41:181-190.

14. Chou JC, Kutter BL, Travasarou T, Chacko JM. Centrifuge modeling of seismically induced uplift for the BART transbay tube. J Geotech Geoenviron Engng 2010; 137(8):754-765.

15. Cilingir U. Seismic response of tunnels. PhD Thesis, Cambridge: University of Cambridge; 2009.

16. Cilingir U, Madabhushi SPG. A model study on the effects of input motion on the seismic behavior of tunnels. Soil Dynam Earthquake Engng 2011a; 31:452-462.

17. Cilingir U, Madabhushi SPG. Effect of depth on the seismic response of square tunnels. Soils Found. 2011b; 51(3):449-457. 
18. Cilingir U, Madabhushi SPG. Effect of depth on the seismic response of circular tunnels. Can Geotech J 2011c; 48(1):117-127.

19. DASYLab. Data Acquisition System Laboratory-User's Manual. Amherst: DASYTEC USA; 2005.

20. FHWA (Federal Highway Administration). Technical manual for design and construction of road tunnels-Civil elements. Publication No. FHWA-NHI-10-034. Washington DC: Department of transportation, Federal Highway Administration; 2009.

21. Ghosh B, Madabhushi SPG An efficient tool for measuring shear wave velocity in the centrifuge. In: Phillips R, Guo PJ, Popescu R, editors. Proceedings of the international conference on physical modelling in geotechnics. Canada: AA Balkema publishers; 2002, p. 119-124.

22. Hardin BO, Drnevich VP. Shear modulus and damping in soil. J Soil Mech Found Div ASCE 1972; 98(7):667-692.

23. Hashash YMA, Hook JJ, Schmidt B, Yao JI-C. Seismic design and analysis of underground structures. Tunnell Underground Space Technol 2001; 16(2):247-293.

24. Hashash YMA, Park D, Yao JI-C. Ovaling deformations of circular tunnels under seismic loading, an update on seismic design and analysis of underground structures. Tunnell Underground Space Technol 2005; 20(5):435-441.

25. Huo H, Bobet A, Fernández G, Ramírez J. Load transfer mechanisms between underground structure and surrounding ground: Evaluation of the failure of the Daikai station. J Geotech Geoenviron Engng 2005; 131(12):1522-1533.

26. Huo H, Bobet A, Fernández G, Ramírez J. Analytical solution for deep rectangular structures subjected to far-field shear stresses. Tunnell Underground Space Technol 2006; 21(6): 613-625.

27. Iida H, Hiroto T, Yoshida N, Iwafuji M. Damage to Daikai subway station. Special issue on geotechnical aspects of the January 171995 Hyogoken-Nanbu earthquake. Soils and Foundations. Tokyo: JSCE, p. 283-300.

28. Ishibashi I, Zhang X. Unified dynamic shear moduli and damping ratios of sand and clay. Soils Found 1993; 33(1):182-191.

29. ISO (International Organization for Standardization) ISO 23469: Bases for design of structures - Seismic actions for designing geotechnical works. International Standard ISO TC98/SC3/WG10. Geneva: International Organization for Standardization; 2005.

30. Kishida $\mathrm{H}$, Uesugi $\mathrm{M}$. Tests of the interface between sand and steel in the simple shear apparatus. Geotechnique 1987; 37(1):45-52.

31. Kontoe S, Zdravkovic L, Potts D, Mentiki C. On the relative merits of simple and advanced constitutive models in dynamic analysis of tunnels. Geotechnique 2011; 61(10):815-829.

32. Kontoe S, Avgerinos V, Potts DM. Numerical validation of analytical solutions and their use for equivalent-linear seismic analysis of circular tunnels. Soil Dynam Earthquake Engng 2014; 66:206-219.

33. Lanzano G, Bilotta E, Russo G, Silvestri F, Madabhushi SPG. Centrifuge modelling of seismic loading on tunnels in sand. Geotech Testing J 2012; 35(6):854-869. 
34. Lanzano G, Bilotta E, Russo G, Silvestri F. Experimental and numerical study on circular tunnels under seismic loading. Eur J Environ Civ Engng 2015; 19(5):539-563.

35. Madabhushi SPG. Centrifuge modelling for civil engineers, CRC Press; 2014.

36. Madabhushi SPG, Schofield AN, Lesley S. A new stored angular momentum (SAM) based actuator. In: Kimura T, Kusakabe O, Takemura J, editors. Proceedings of the international conference centrifuge 98. Tokyo: AA Balkema publishers; 1998, p. 111-116.

37. Madabhushi SPG, Houghton NE, Haigh SK. A new automatic sand pourer for model preparation at University of Cambridge. In: Ng CWW, Wang YH, Zhang LM, editors. Proceedings of the sixth international conference on physical modelling in geotechnics. London: Taylor and Francis Group; 2006, p. 217-222.

38. Mononobe N, Matsuo H. On the determination of earth pressures during earthquakes. In: Proceedings of the Second World Conference on Earthquake Engineering, Tokyo, Japan, 1929.

39. Okabe S. General theory of earth pressure and laboratory testings on seismic stability of retaining walls. Proc. JSCE 1926; 12(1):123-134.

40. Penzien J. Seismically induced racking of tunnel linings. Earthquake Engng Structl Dynamics 2008; 29:683-691.

41. Pistolas GA, Tsinaris A, Anastasiadis A, Pitilakis K. Undrained dynamic properties of Hostun sand. Proceedings of $7^{\text {th }}$ Greek Geotechnics Conference. Athens: Greece Hellenic Society of Soil Mechanics and Geotechnical Engineering; 2014 (in Greek).

42. Pitilakis K, Tsinidis G. Performance and seismic design of underground structures. In: Maugeri M, Soccodato C, editors. Earthquake geotechnical engineering design, Geotechnical Geological and Earthquake Engineering 28. Switzerland: Springer international publishing: 2014, p. 279-340.

43. Schanz T, Vermeer PA Angles of friction and dilatancy of sand. Geotechnique 1996; 46(1):145-151.

44. Schofield AN. Dynamic and earthquake centrifuge modelling. Proceedings of the international conference on recent advances in geotechnical earthquake engineering and soil dynamics. University of Missouri-Rolla, 1981, p. 1081-1100.

45. Seed H, Wong R, Idriss I, Tokimatsu K. Moduli and damping factors for dynamic analyses of cohesionless soils. J Geotech Engng ASCE 1984; 112(11):1016-1032.

46. Shibayama S, Izawa J, Takahashi A, Takemura J, Kusakabe O. Observed behavior of a tunnel in sand subjected to shear deformation in a centrifuge. Soils found 2010; 50(2):281-294.

47. Tsinidis G, Heron C, Pitilakis K, Madabhushi SPG. Physical modeling for the evaluation of the seismic behavior of square tunnels. In: Ilki A, Fardis M, editors. Seismic evaluation and rehabilitation of structures, Geotechnical Geological and Earthquake Engineering, 26. Switzerland: Springer International Publishing; 2014a, p. 389-406.

48. Tsinidis G, Pitilakis K, Trikalioti AD. Numerical simulation of round robin numerical test on tunnels using a simplified kinematic hardening model. Acta Geotechnica, 2014b; 9(4):641-659.

49. Tsinidis G. On the seismic behavior and design of tunnel. $\mathrm{PhD}$ thesis. Aristotle University, Thessaloniki, 
50. Tsinidis G, Rovithis E, Pitilakis K, Chazelas JL. Dynamic response of shallow rectangular tunnels in sand by centrifuge testing. In: Taucer F, Apostolska R, editors. Experimental research in earthquake engineering - EU-SERIES concluding workshop, Geotechnical Geological and Earthquake Engineering, 35. Switzerland: Springer International Publishing: 2015a, p. 493-507.

51. Tsinidis G, Heron C, Madabhushi SPG, Pitilakis K. Calibration of Strain Gauged Square Tunnels for Centrifuge Testing. Geotech Geol Eng 2016; 34: 911-921.

52. Ulgen D, Saglam S, Ozkan MY. Dynamic response of a flexible rectangular underground structure in sand: centrifuge modeling. Bull Earthquake Engng 2015; 13:2547-2566.

53. Wang JN. Seismic design of tunnels: A simple state of the art design approach. New York: Parsons Brinckerhoff; 1993.

54. Zeghal M, Elgamal AW. Analysis of site liquefaction using earthquake records. J Geotech Engng ASCE 1994; 120(6):996-1017.

55. Zeng X, Schofield AN. Design and performance of an equivalent shear beam (ESB) container for earthquake centrifuge modelling. Geotechnique 1996; 46(1):83-102.

56. Zhao Y, Gafar K, Elshafie MZEB, Deeks AD, Knappett JA, Madabhushi SPG. Calibration and use of a new automatic sand pourer. In: Ng CWW, Wang YH, Zhang LM, editors. Proceedings of the sixth p. 265-270. 
Click here to download Figure: Tsinidisetal.fig.1.eps

(a)

(b)

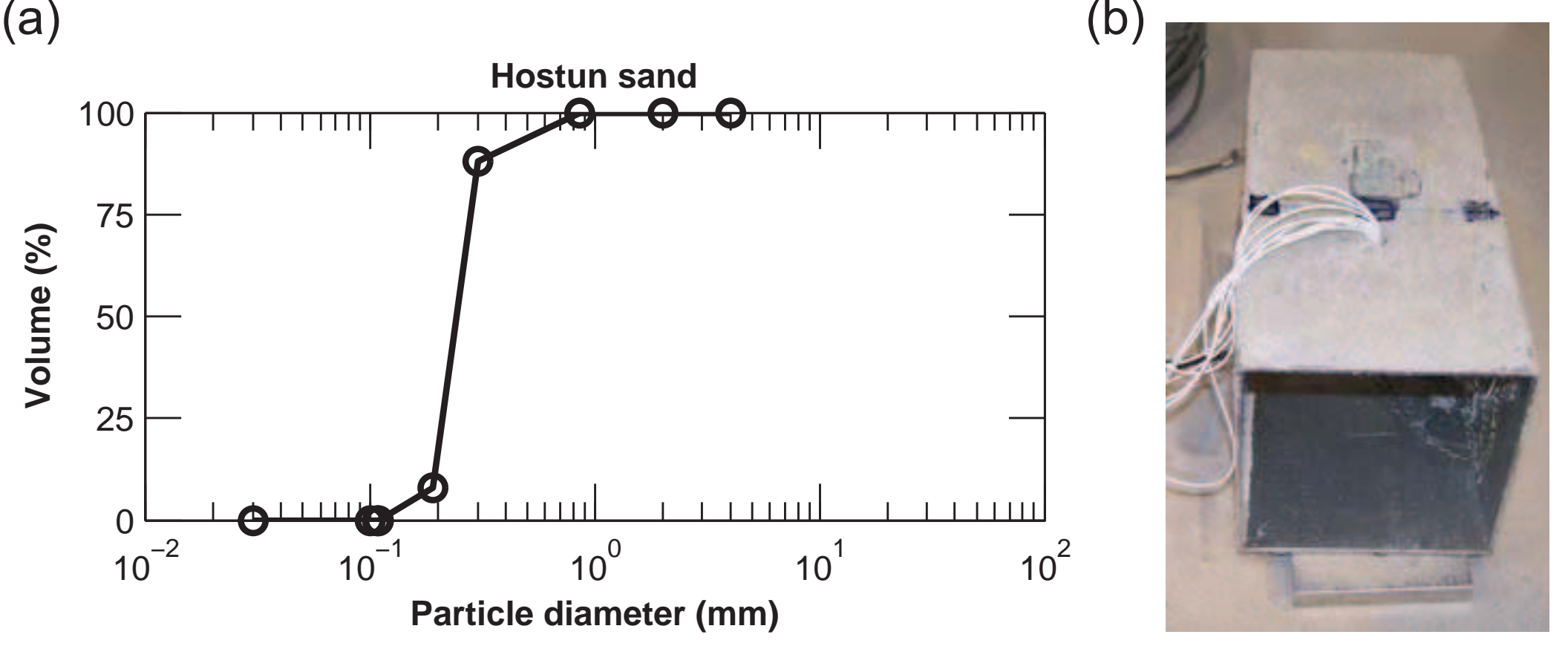




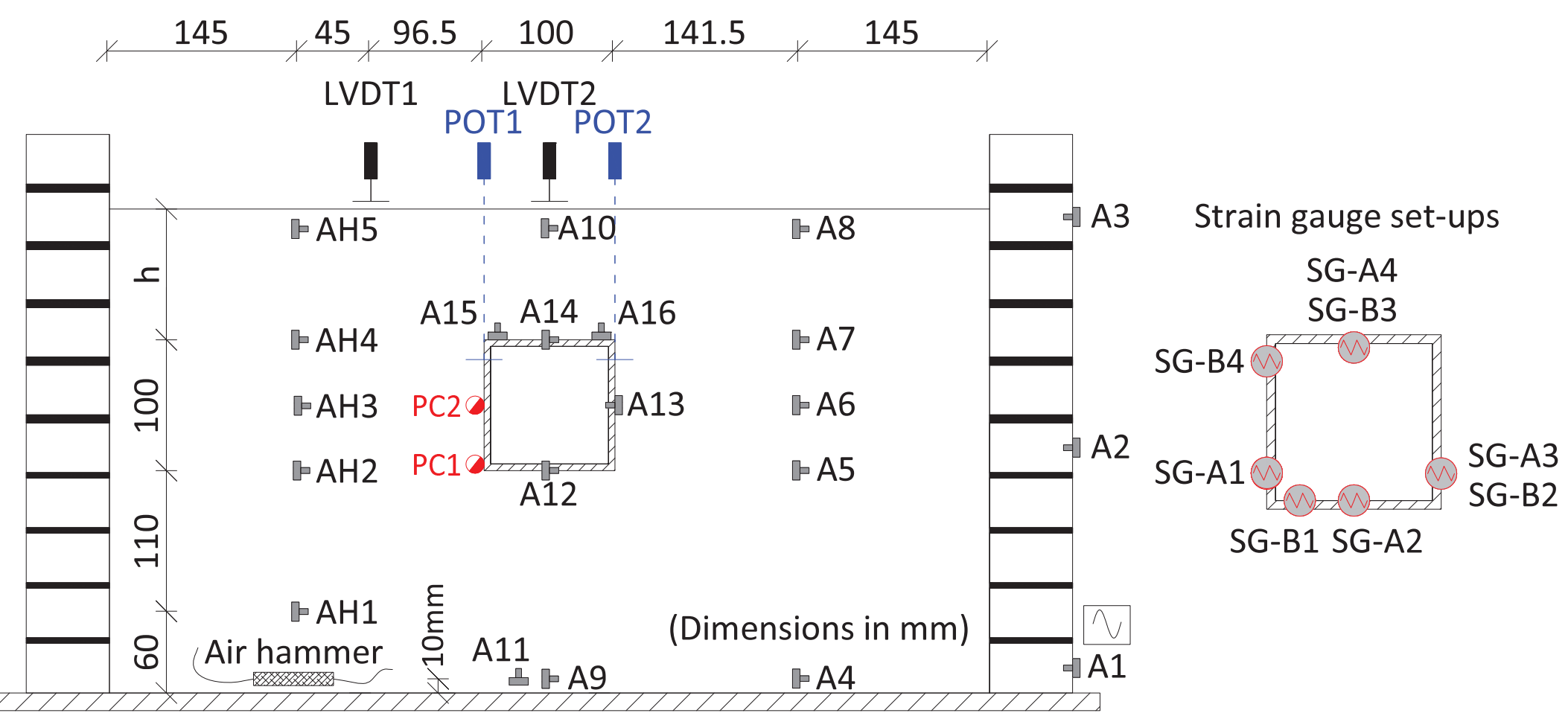

$\triangle$ Accelerometer Pressure cell $\square$ LVDT POT Strain gauge 
Beam elements +
Soil-tunnel interface

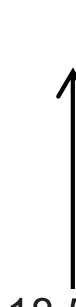

18
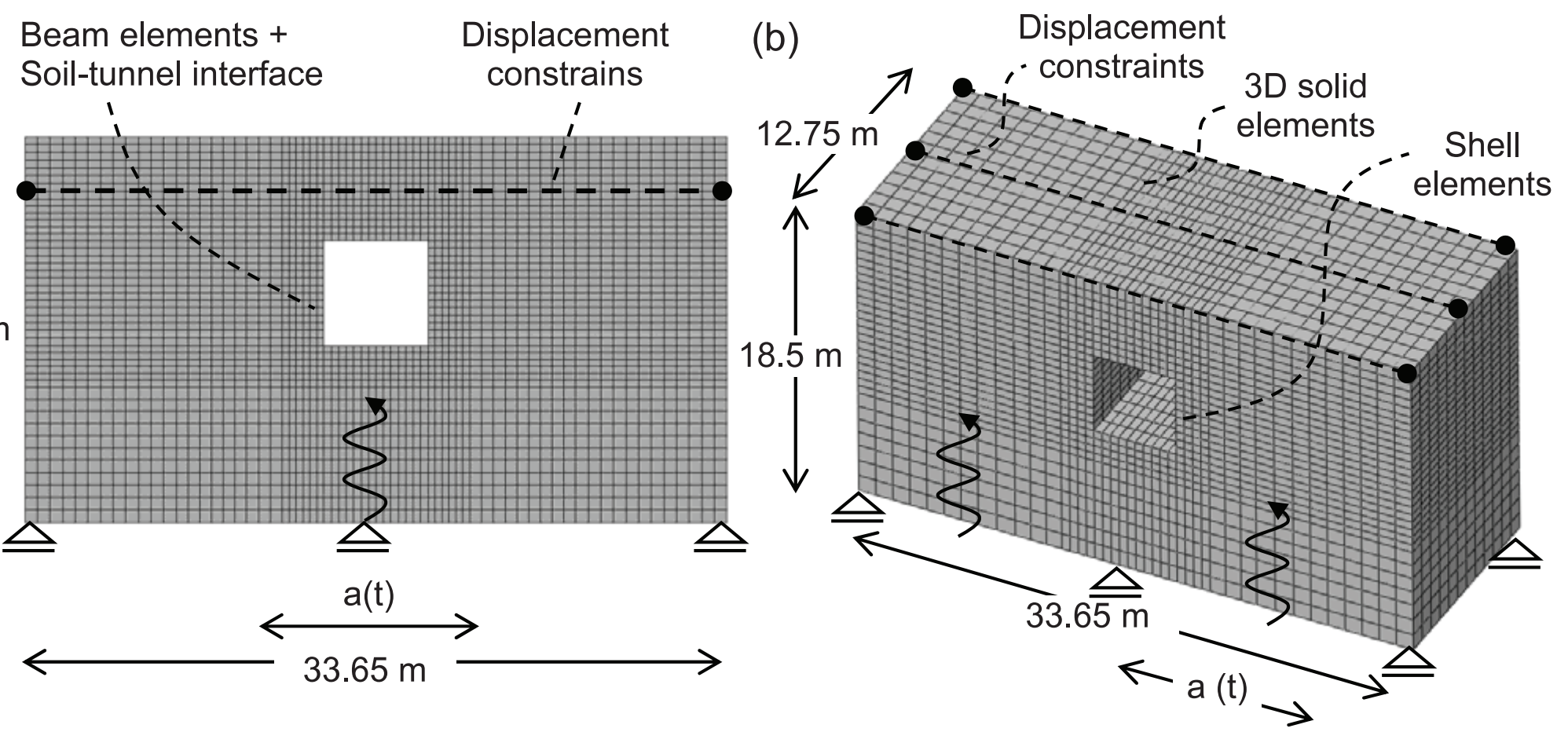
Figure 6
Click here to download Figure: Tsinidisetal.fig.6.eps

(a)

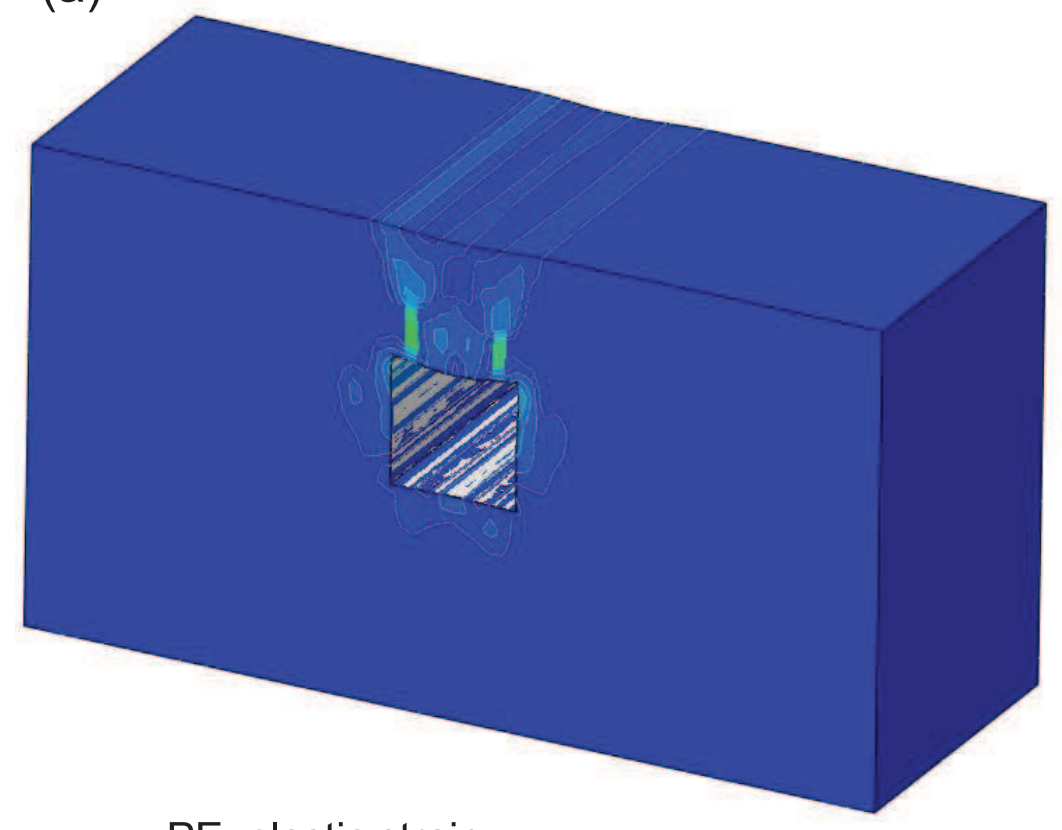

$\mathrm{PE}$, plastic strain

0.007

0.006

0.005

(b)

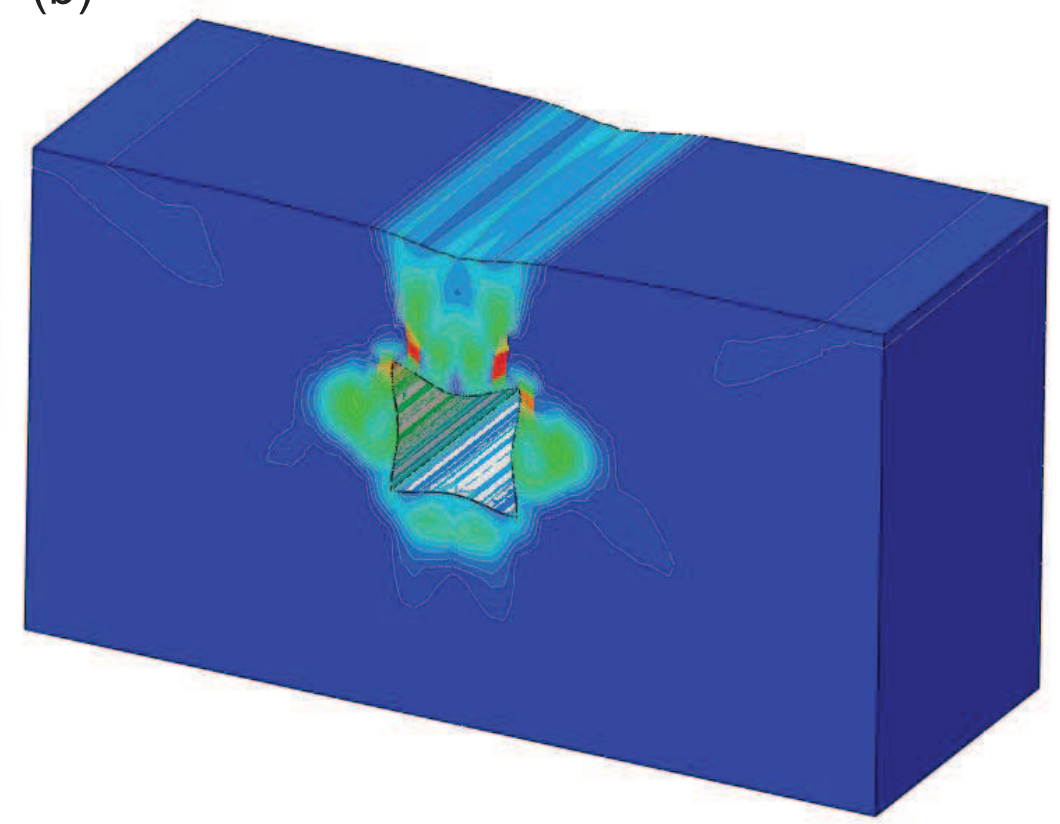

$0.004 \quad 0.003$

0.002

0.001

0.000 
(a)
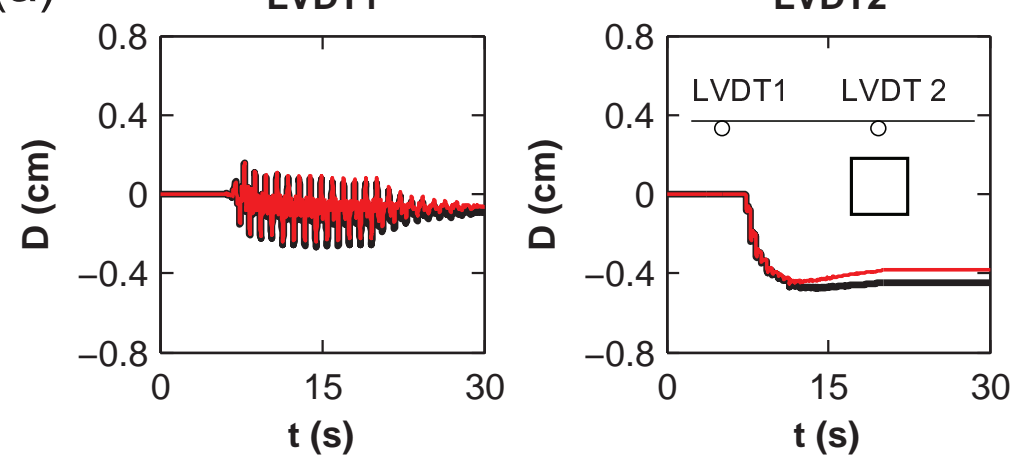

(b)
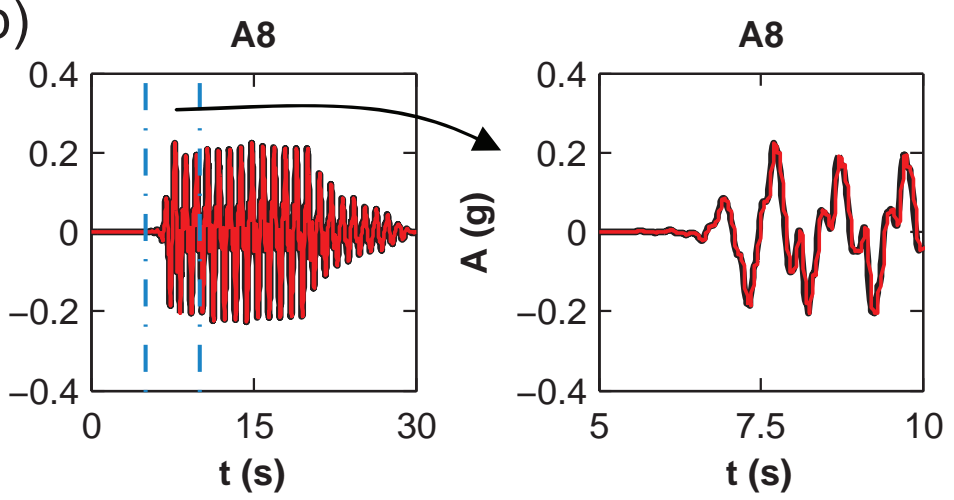

(c)
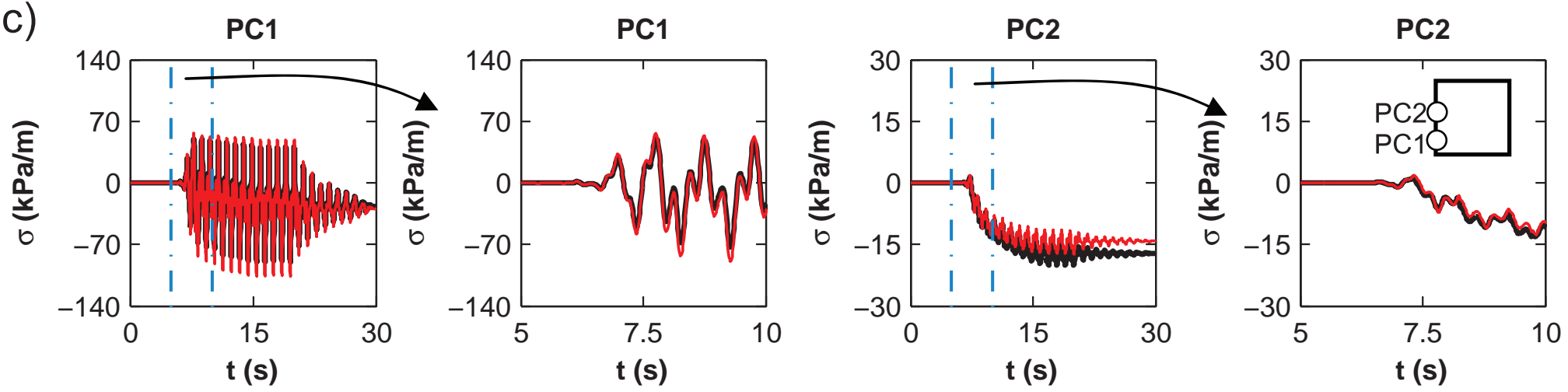

(d)
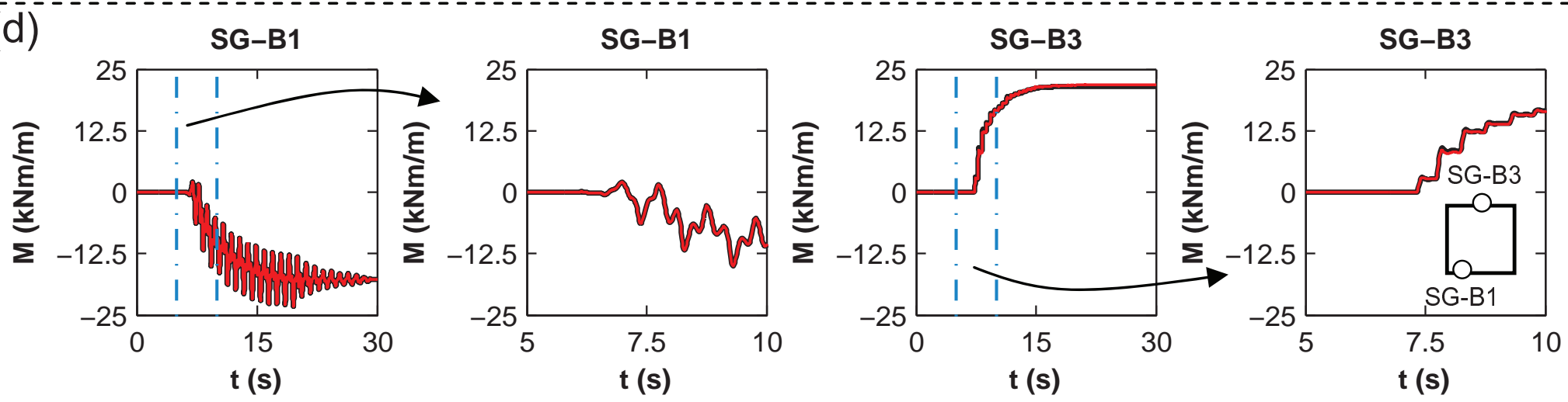

(e

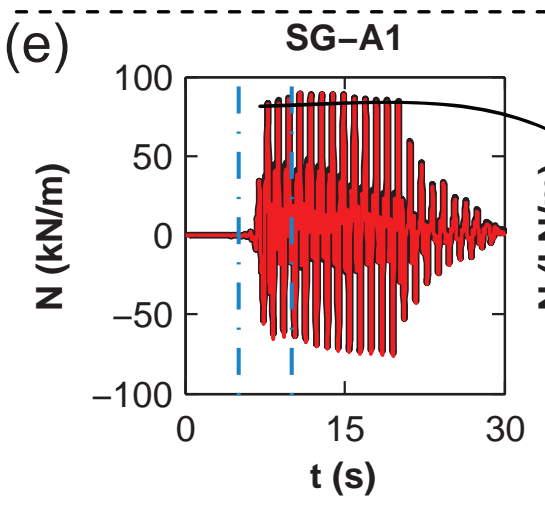

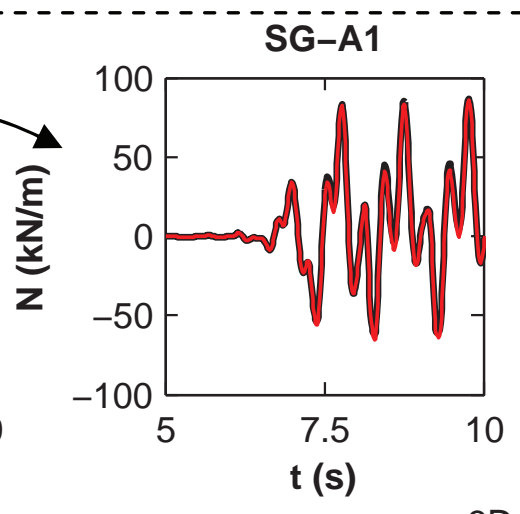
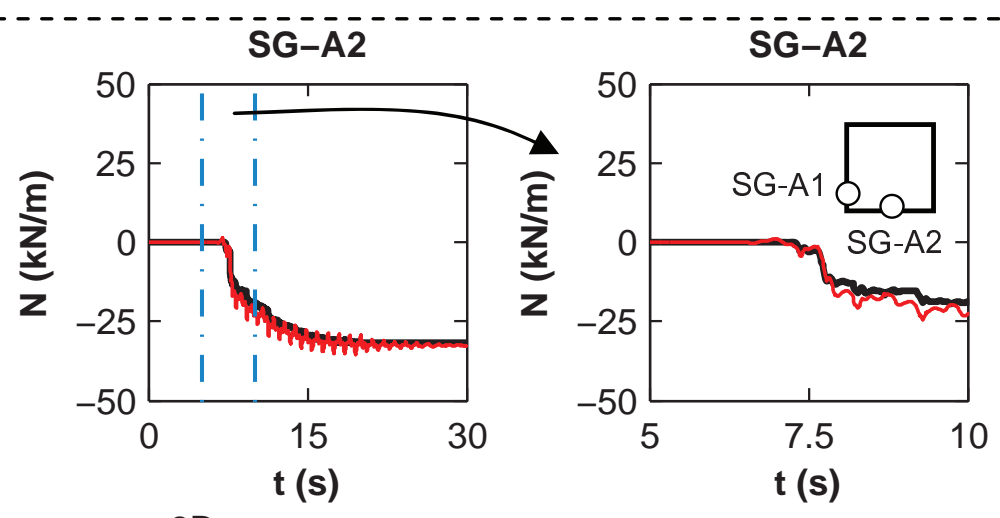
(a)

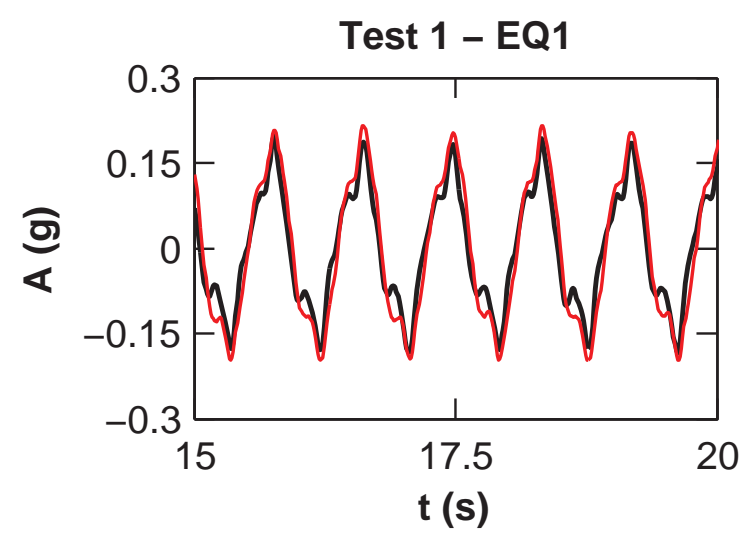

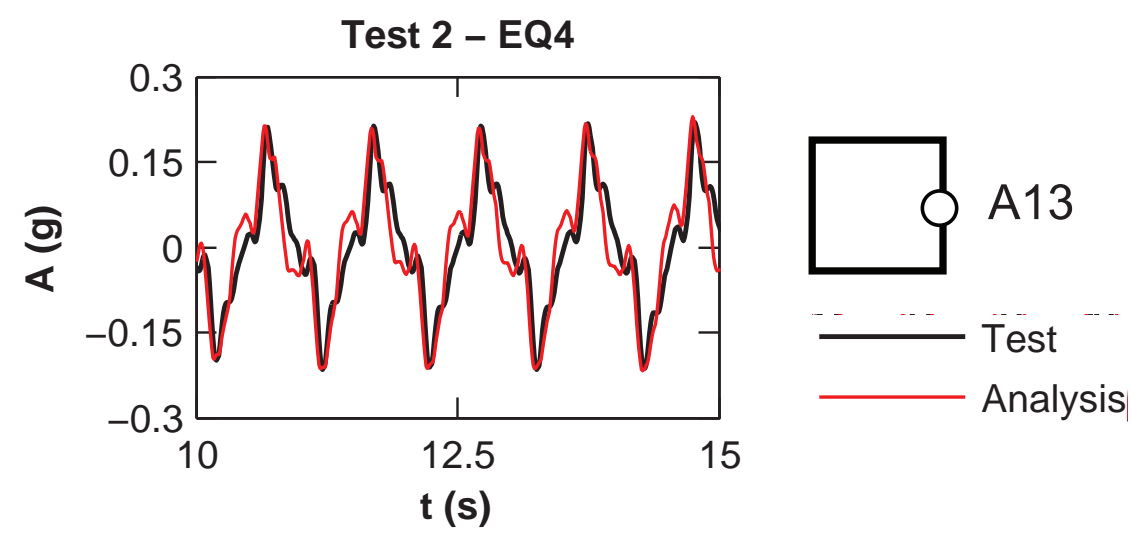

气

Reference array - A (g)

Free field array - A (g)

Tunnel array - A (g)
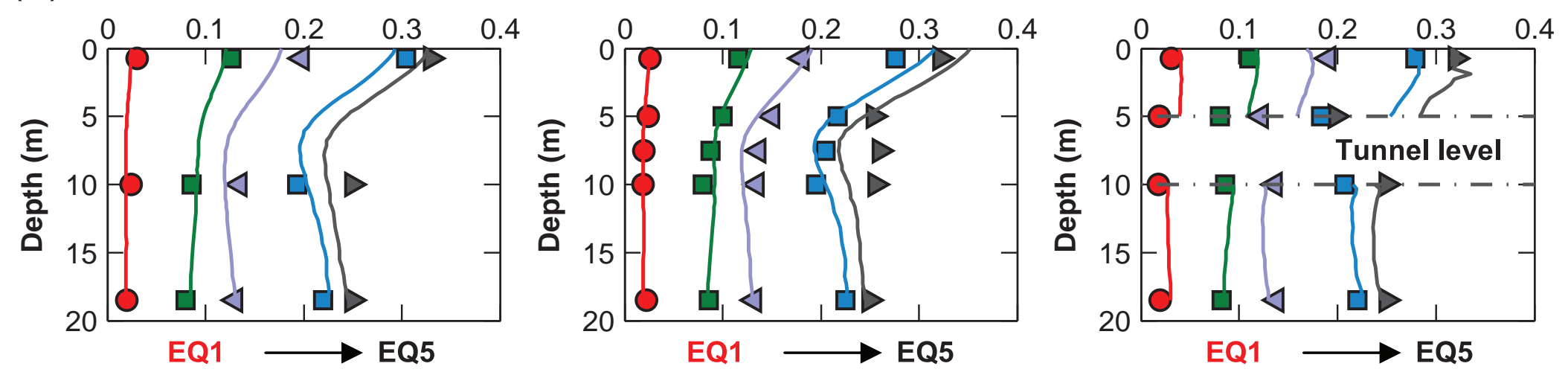
(a)
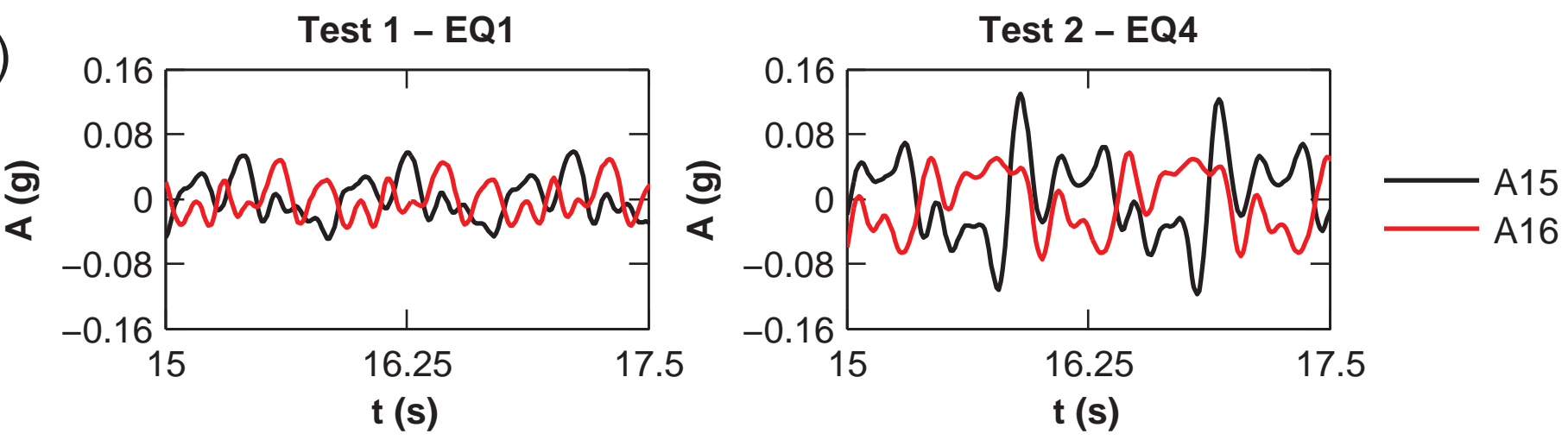

A15 A16

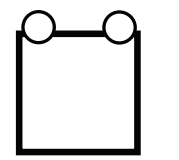

(b)

Flexible tunnel Flexible tunnel

Elastic soil Elasto-plastic soil
Rigid tunnel

Elastic soil
Rigid tunnel

Elasto-plastic soil
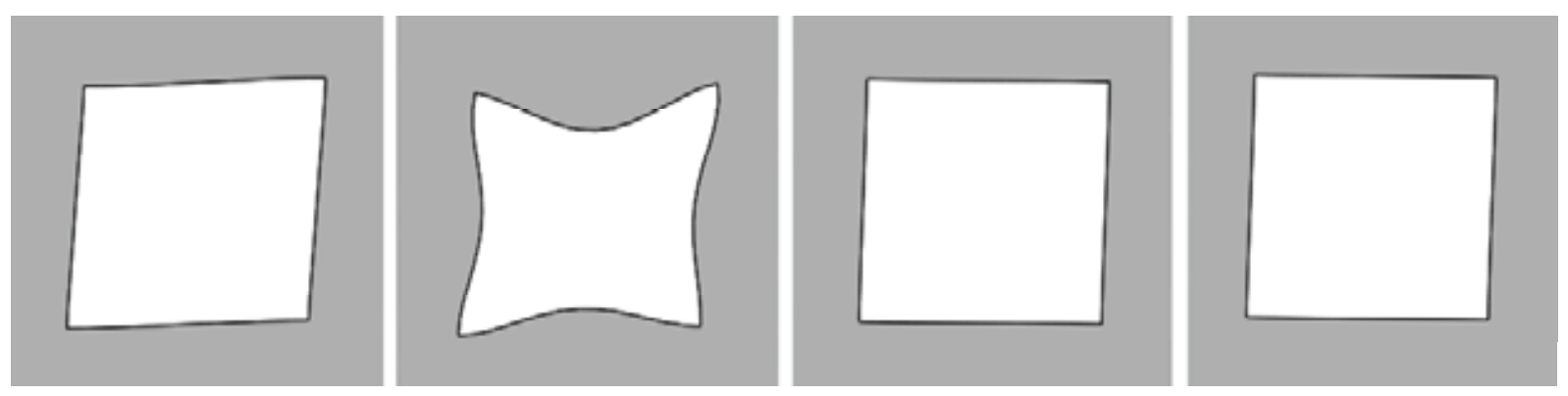
(a)
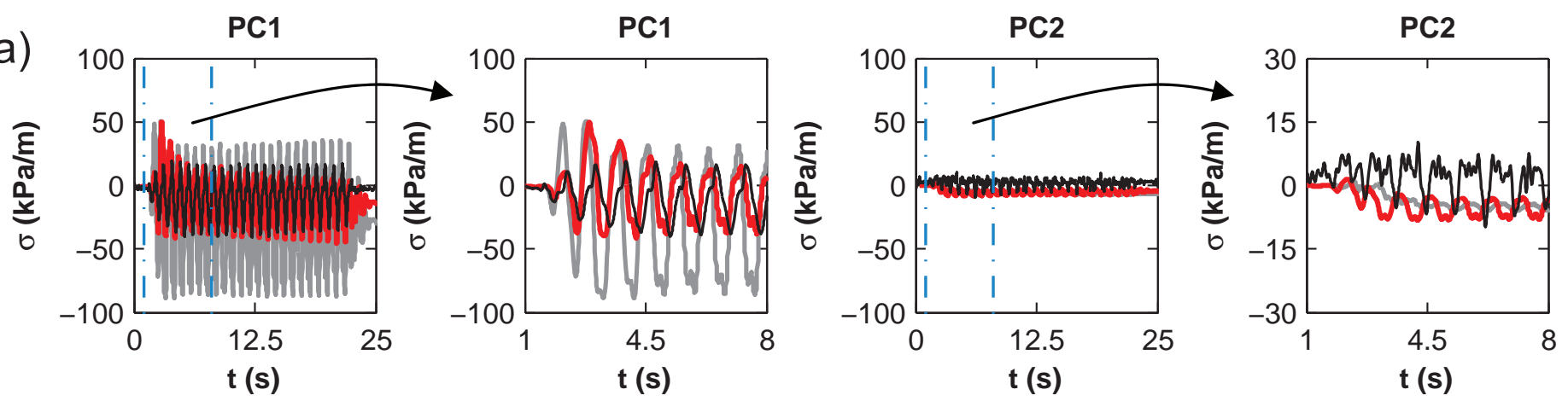

(b)
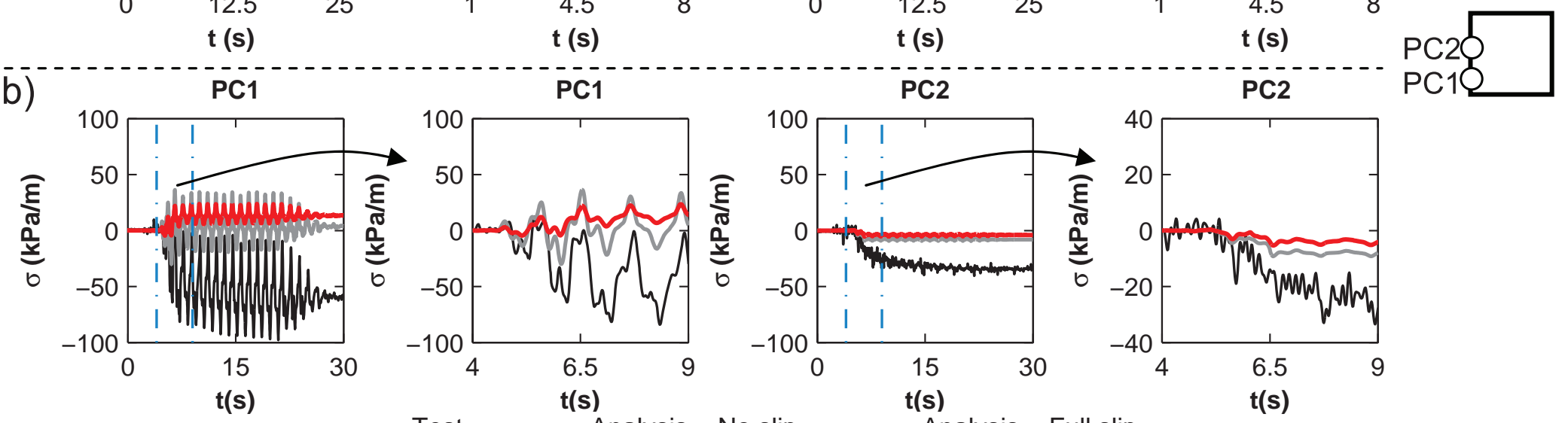


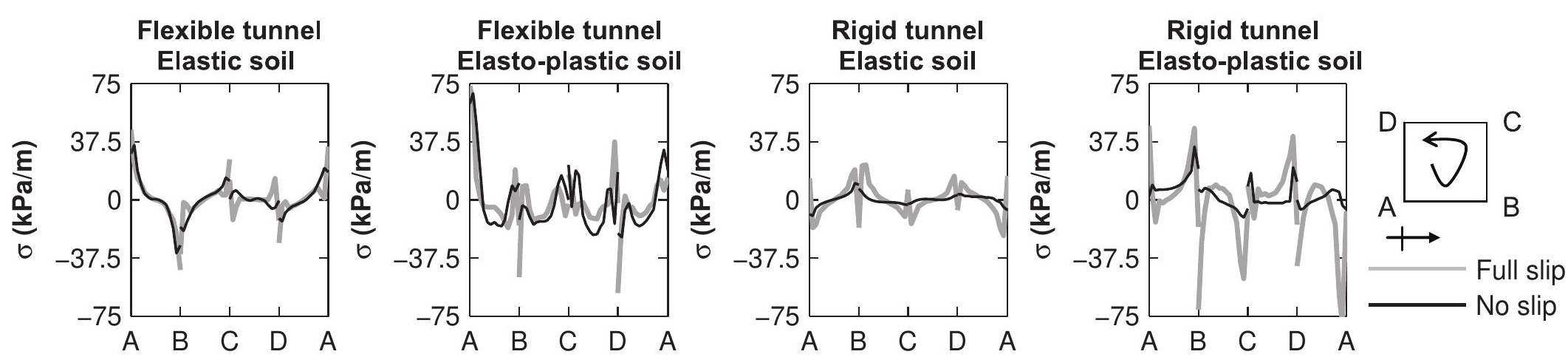


(a)

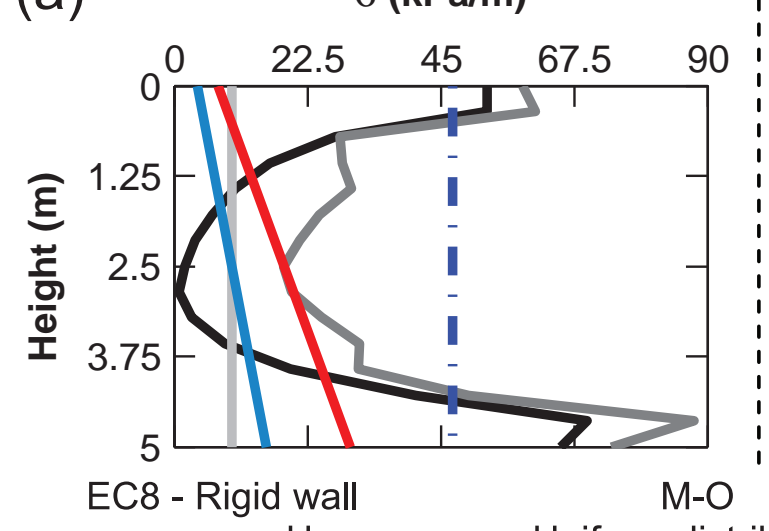

$\sigma=\alpha \times y \times H$

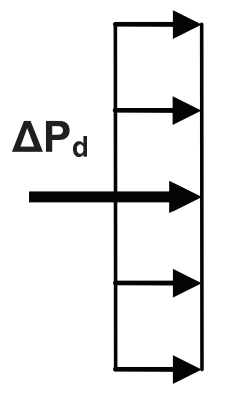

(b)

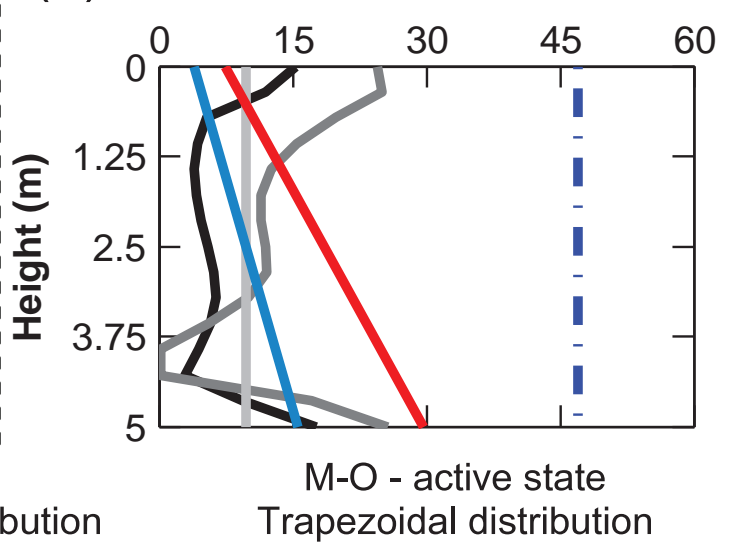

Analysis - Elastic

Analysis - Elasto-Plastic

M-O - Uniform

- M-O - Triangular

1 - ' - EC8 - Rigid wall

M-O - Passive

M-O - passive state Trapezoidal distribution
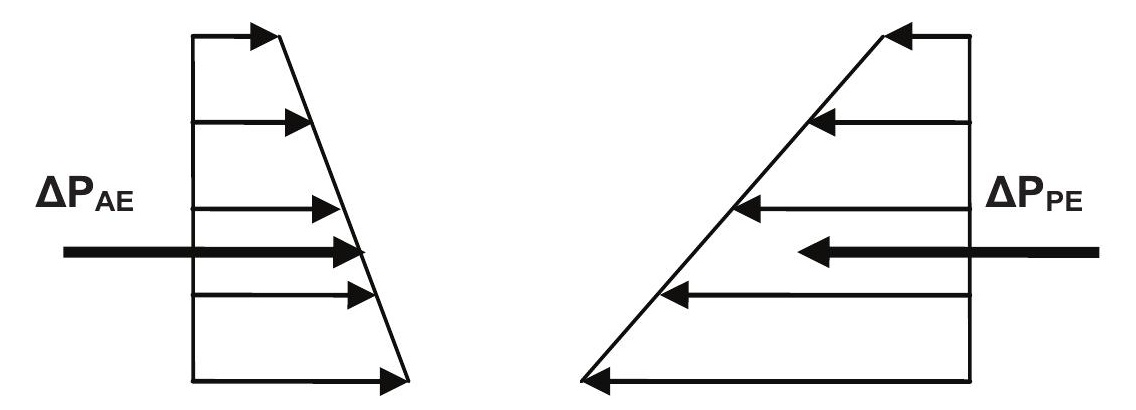
Flexible tunnel Elastic soil

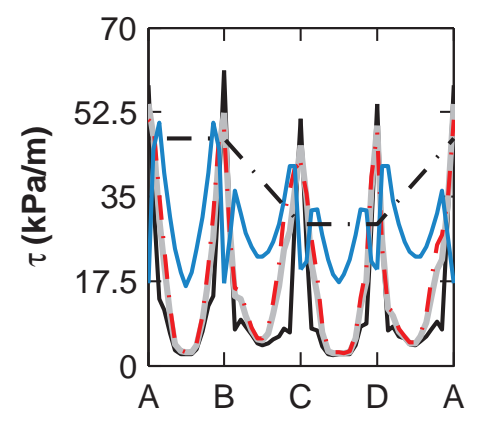

Flexible tunnel Elasto-plastic soil

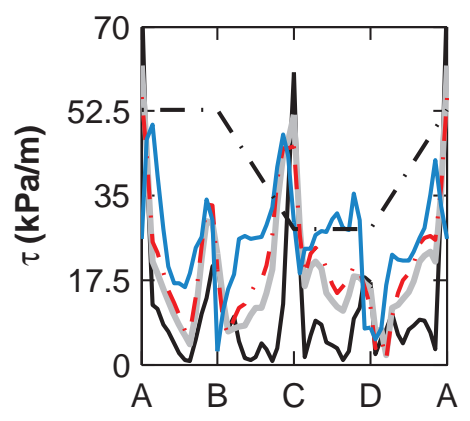

Rigid tunnel Elastic soil

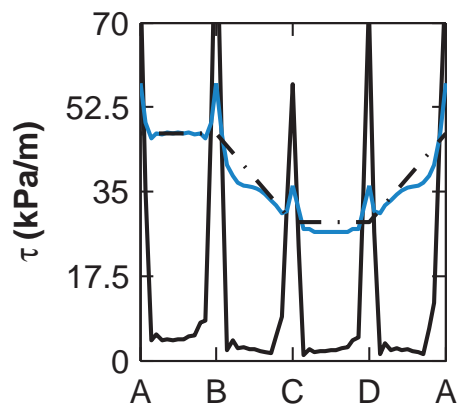

Rigid tunnel Elasto-plastic soil

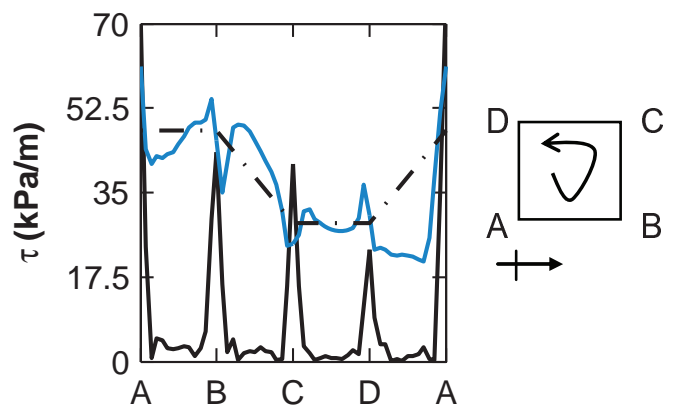

Full slip $\quad \mu=0.4 \cdot-\cdot, \mu=0.8 \longrightarrow$ No slip $\cdot-$ Soil free field 
(a)
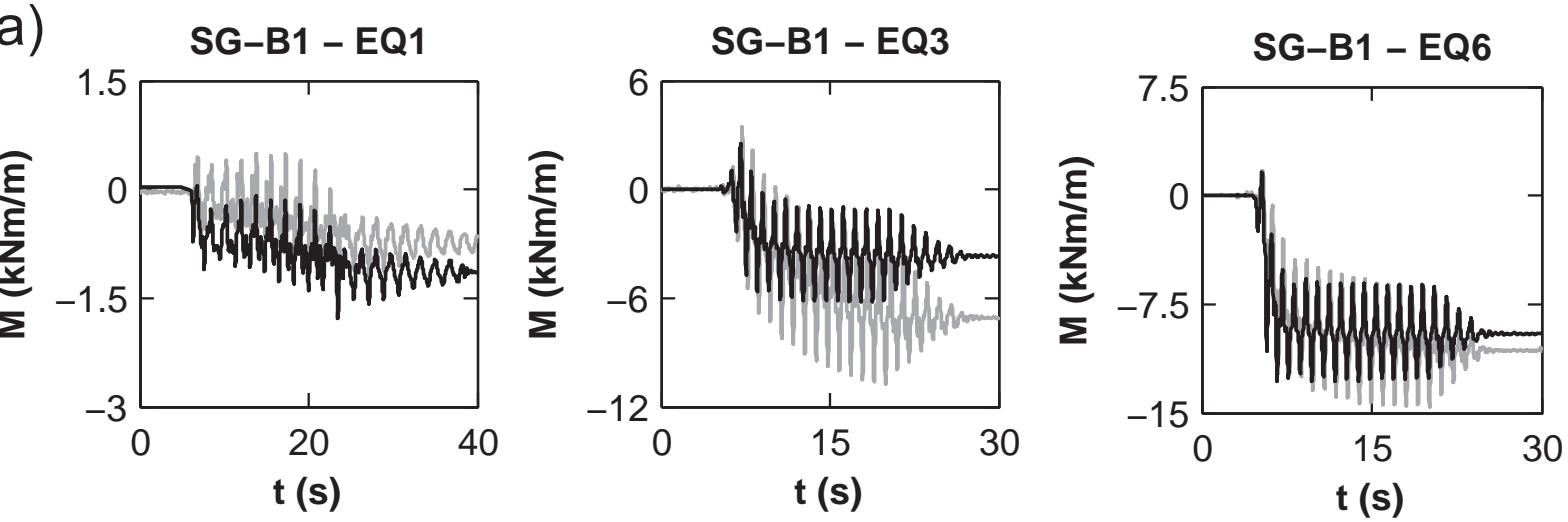

Test

Analysis

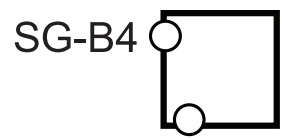

SG-B1

(b)
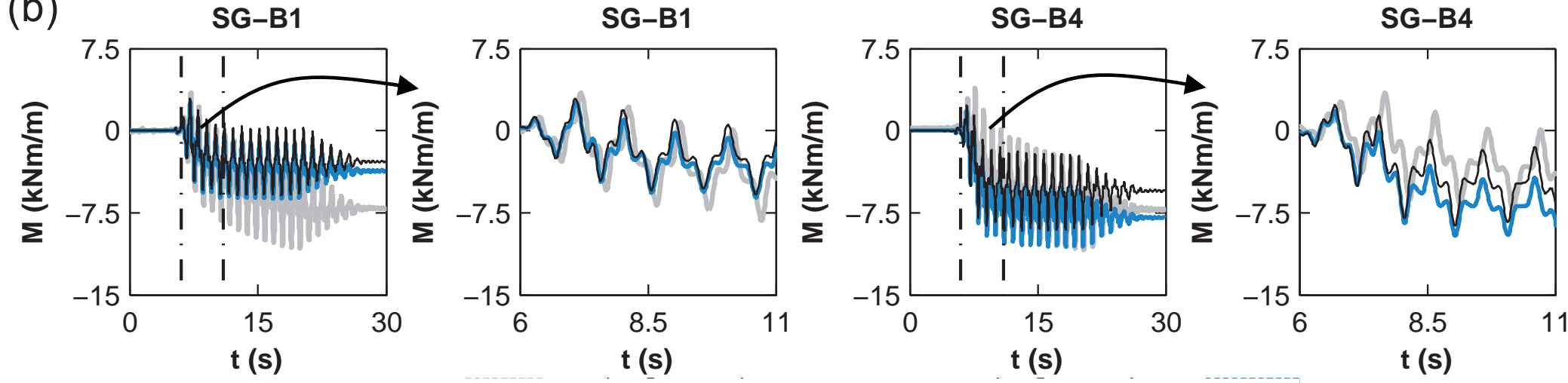

Test

Analysis - No slip

Analysis - Full slip

(c)

है

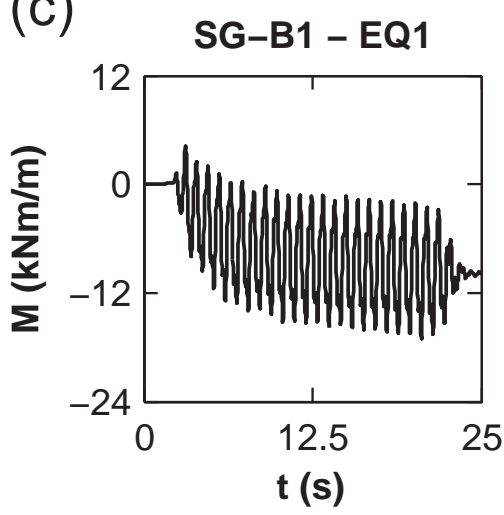

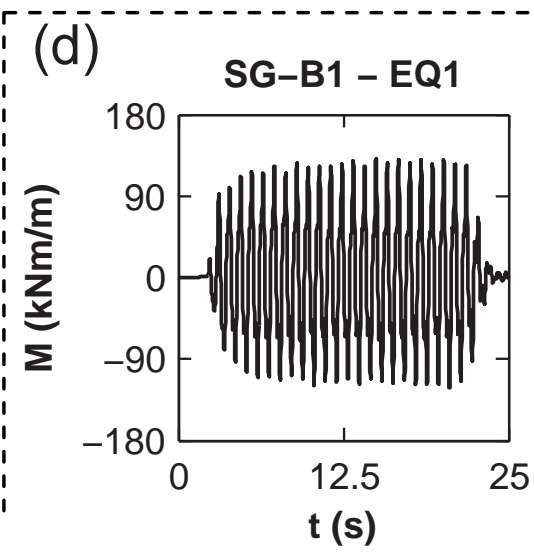



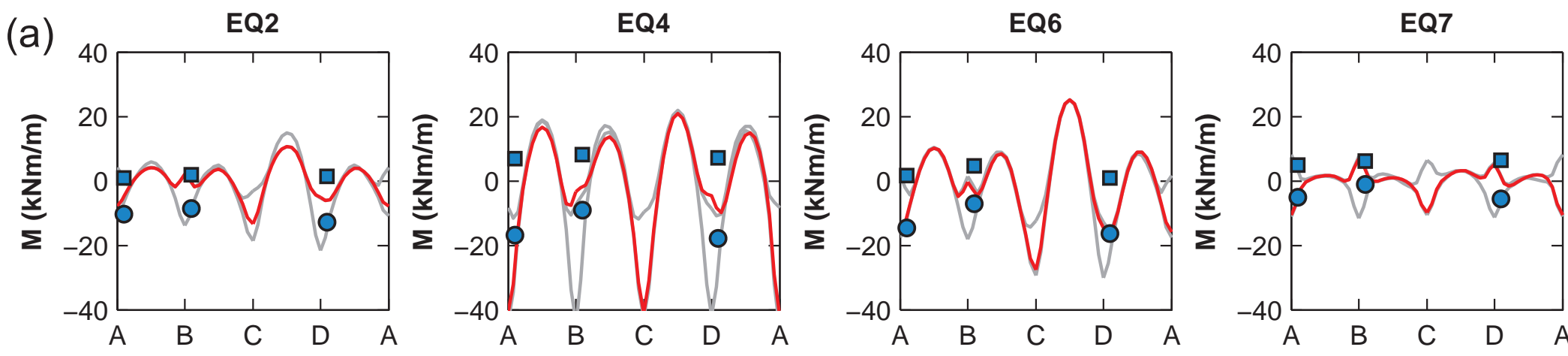

Analysis - Envelope

$$
\text { Analysis - Racking }
$$

ㅁ Test - Maximum

- Test - Minimum

(b)

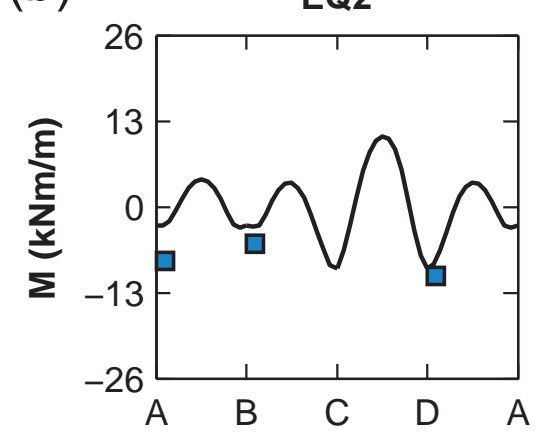

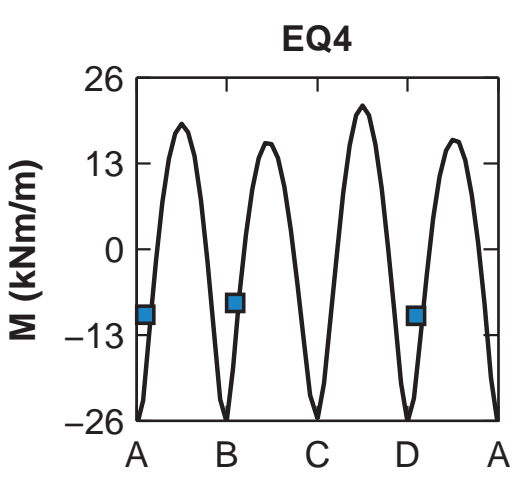
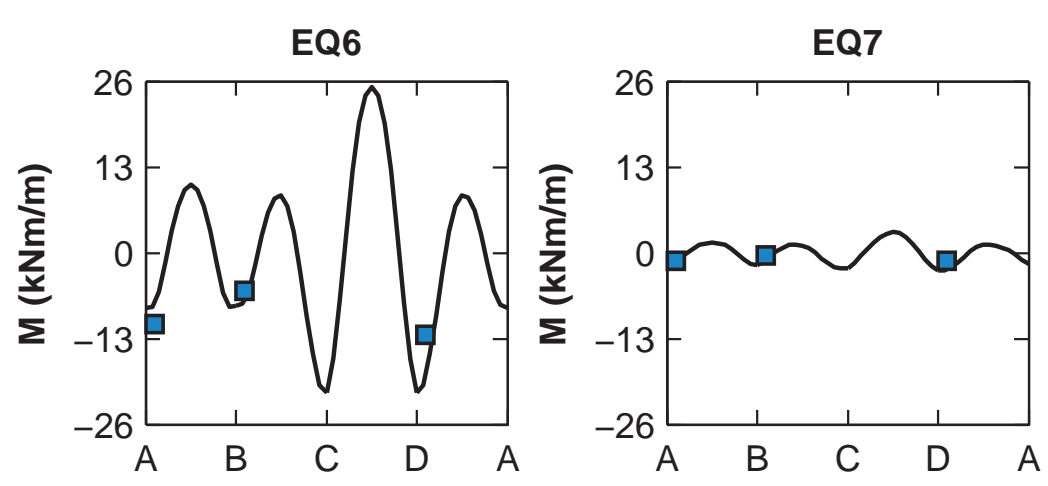

Analysis $\square$ Test

(c)

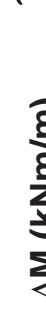

EQ2
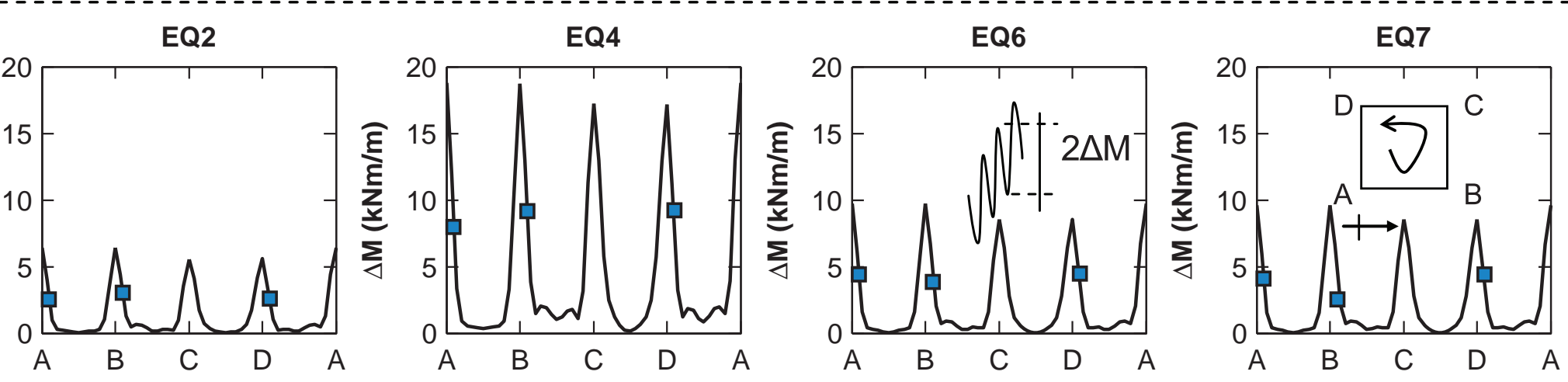

Analysis 
$(a$
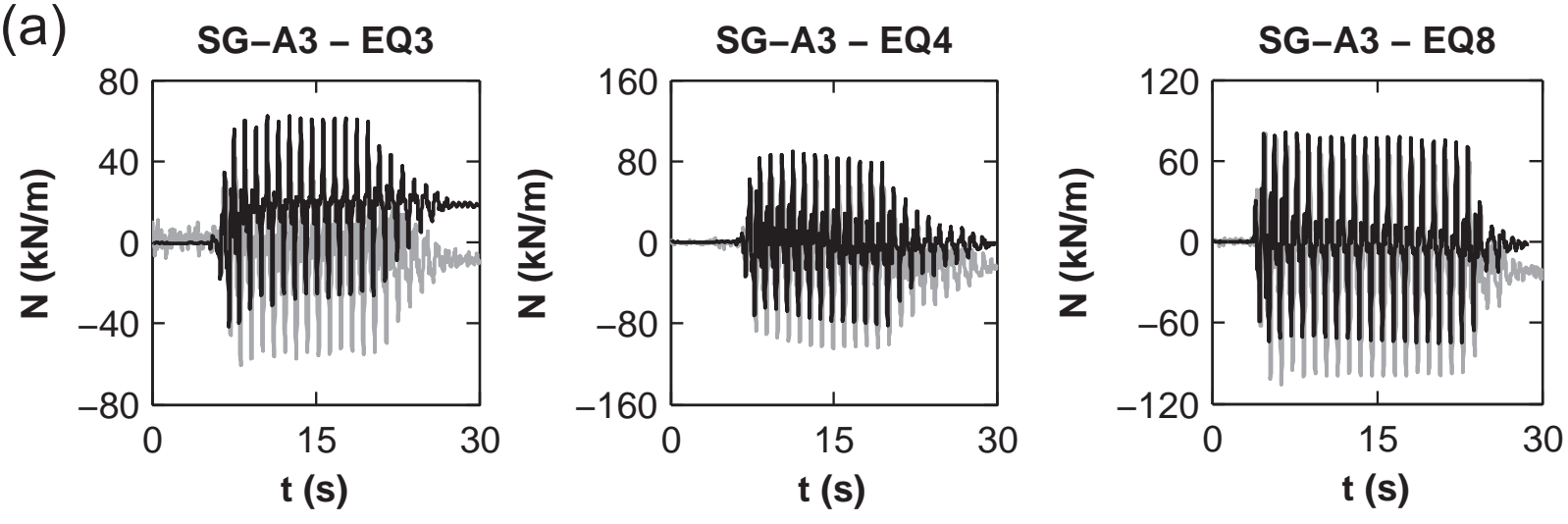

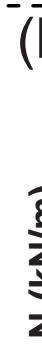
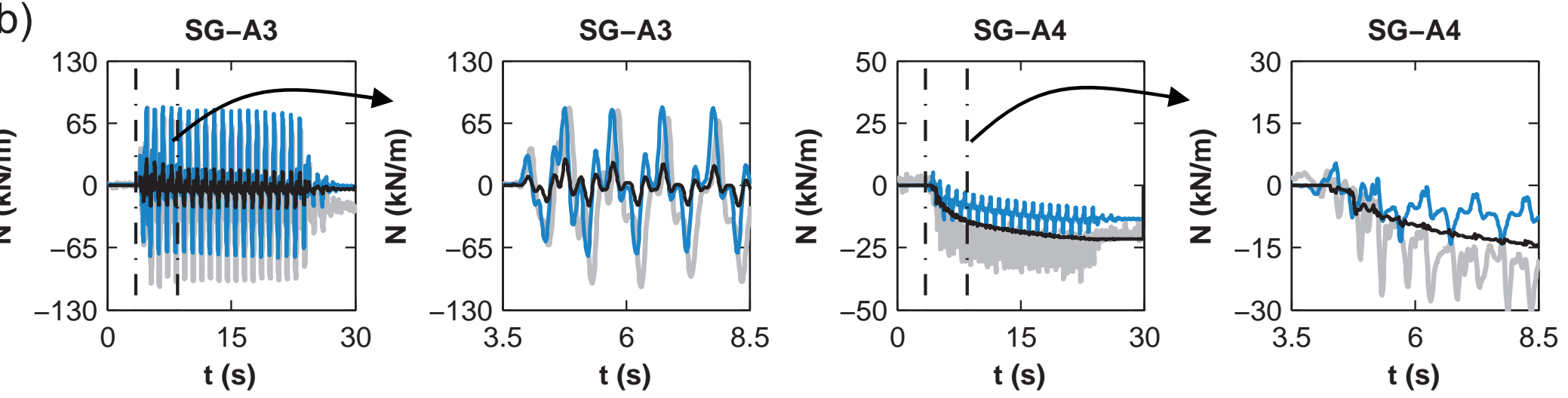

Test

- Analysis - No slip

Analysis - Full slip

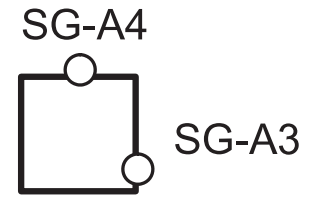

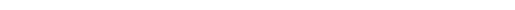


(a

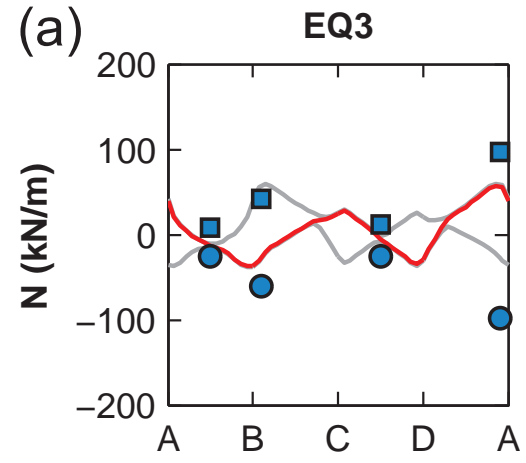

EQ4

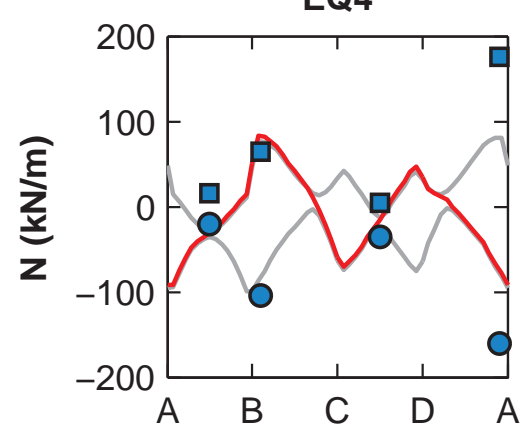

EQ5

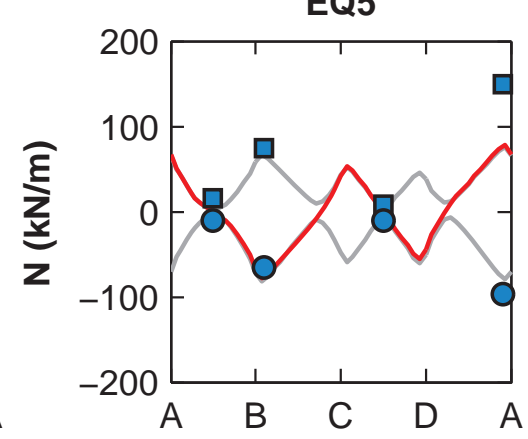

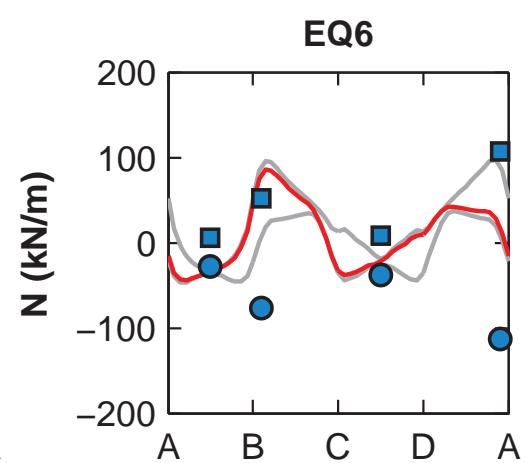

Analysis - Envelope

Analysis - Racking

- Test-Maximum

- Test - Minimum

(a)

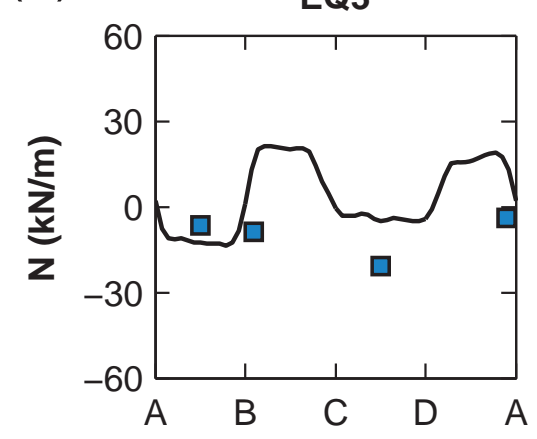

EQ4

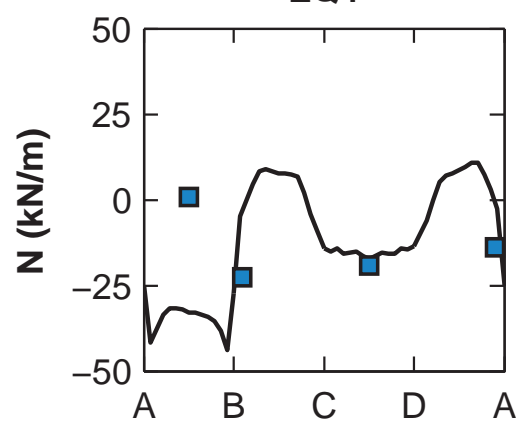

EQ5

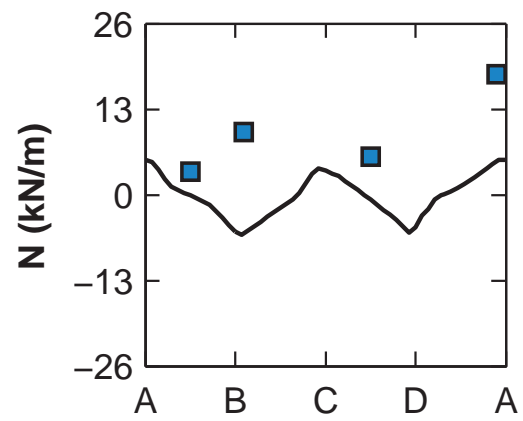

EQ6

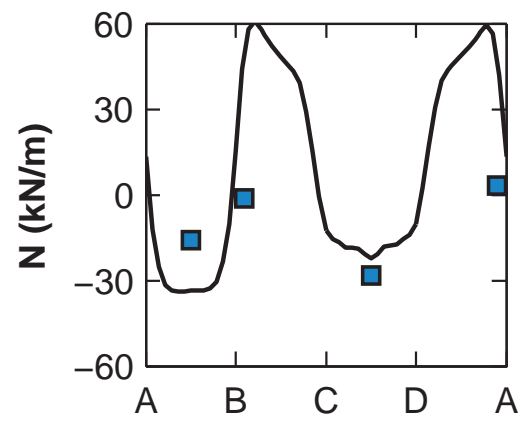

Analysis $\quad$ Test

(c

(C)

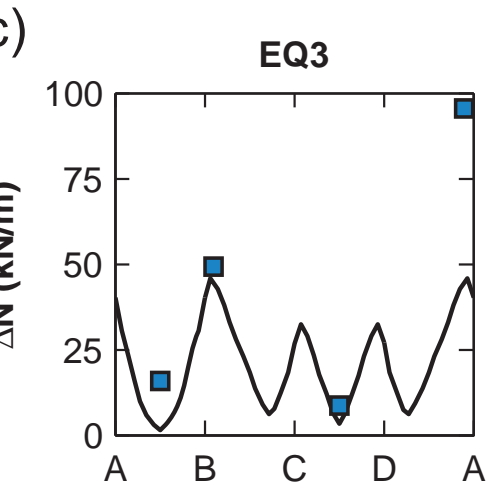

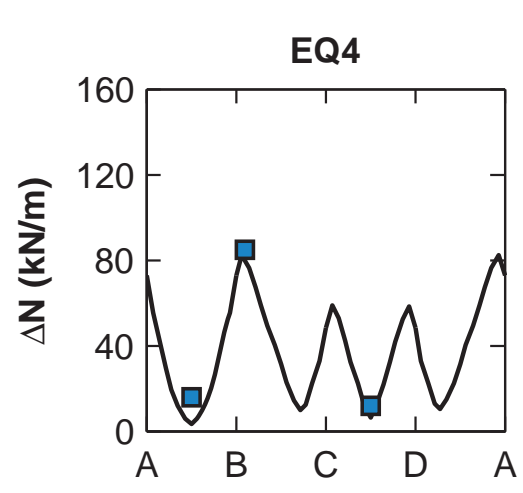

EQ6

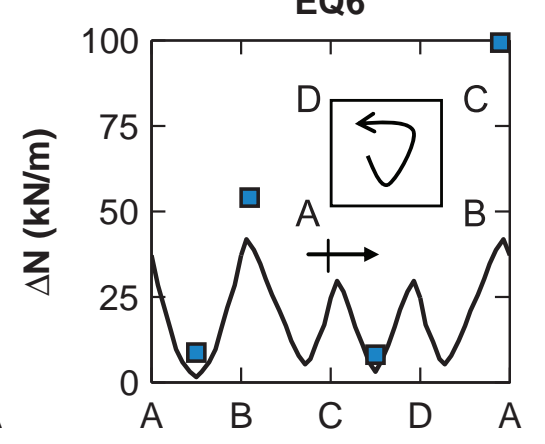

EQ5

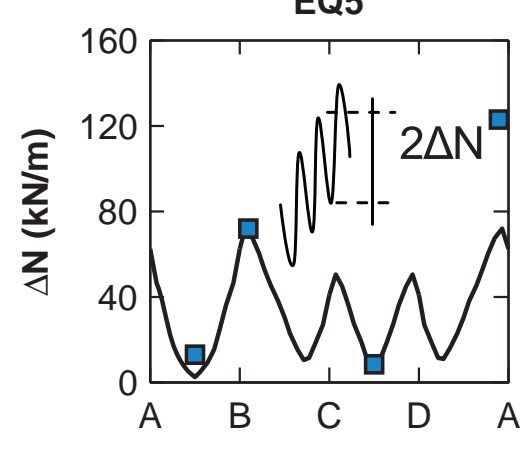


(a)
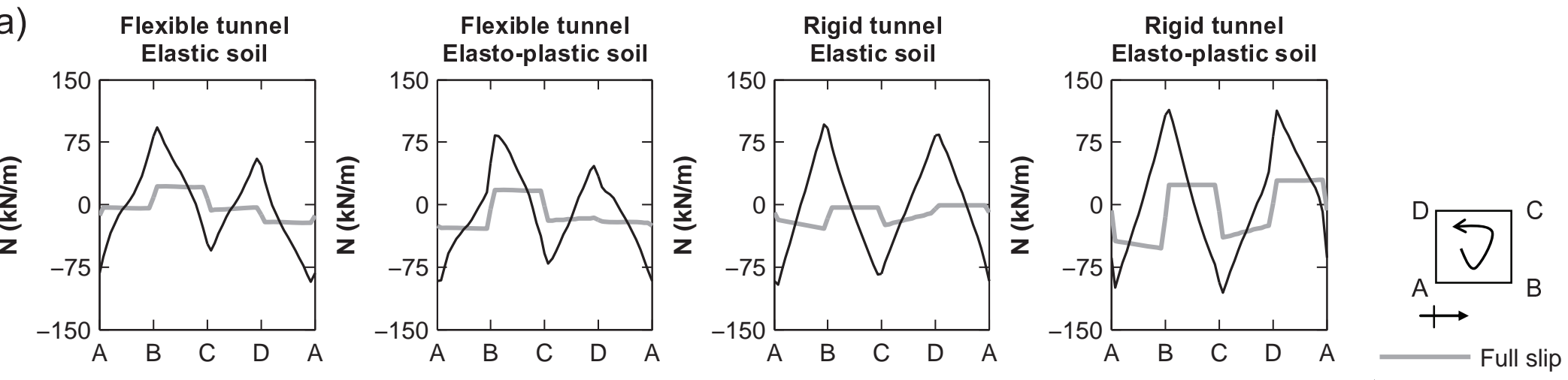

(b

Flexible tunnel Elastic soil

3

そ̇

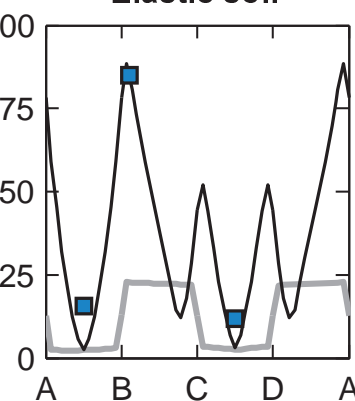

Flexible tunnel Elasto-plastic soil

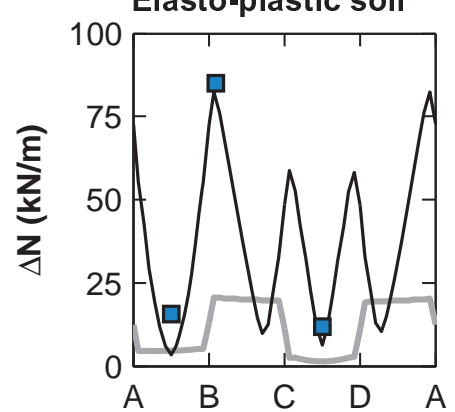

Rigid tunnel

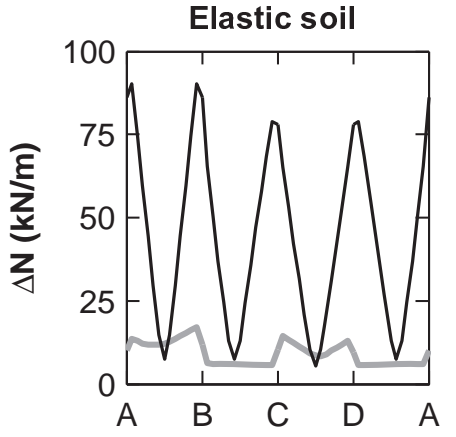

Elasto-plastic soil

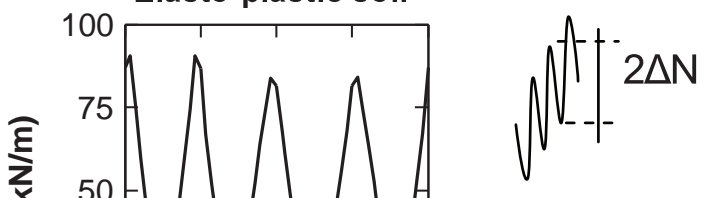

